DFAQ-99/TH/06

DFPD-99/TH/29

March 2000

\title{
Predictive Grand Unified Textures for Quark and Neutrino Masses and Mixings
}

\author{
Zurab Berezhiani ${ }^{a, b, \text {, and Anna Rossi }}{ }^{c, \text {, }}$ \\ a Dipartimento di Fisica, Università di L'Aquila, I-67010 Coppito, AQ, and \\ INFN, Laboratori Nazionali del Gran Sasso, I-67010 Assergi, AQ, Italy \\ ${ }^{b}$ The Andronikashvili Institute of Physics, Georgian Academy of Sciences, \\ 38007 ry Tbilisi, Georgia \\ ${ }^{c}$ Dipartimento di Fisica, Università di Padova and \\ INFN Sezione di Padova, I-35131 Padova, Italy.
}

\begin{abstract}
We propose new textures for the fermion Yukawa matrices which are generalizations of the so-called Stech ansatz. We discuss how these textures can be realized in supersymmetric grand unified models with horizontal symmetry $S U(3)_{H}$ among the fermion generations. In this framework the mass and mixing hierarchy of fermions (including neutrinos) can emerge in a natural way. We emphasize the central role played by the $S U(3)_{H}$ adjoint Higgs field which reduces $S U(3)_{H}$ to $U(2)_{H}$ at the GUT scale. A complete $S O(10) \times S U(3)_{H}$ model is presented in which the desired Yukawa textures can be obtained by symmetry reasons. The phenomenological implications of these textures are thoroughly investigated. Among various realistic possibilities for the Clebsch factors between the quark and lepton entries, we find three different solutions which provide excellent fits of the quark masses and CKM mixing angles. Interestingly, all these solutions predict the correct amount of $\mathrm{CP}$ violation via the CKM mechanism, and, in addition, lead to an appealing pattern of the neutrino masses and mixing angles. In particular, they all predict nearly maximal 23 mixing and small 12 mixing in the lepton sector, respectively in the range needed for the explanation of the atmospheric and solar neutrino anomaly.
\end{abstract}

\footnotetext{
${ }^{1}$ E-mail address: berezhiani@fe.infn.it

${ }^{2}$ E-mail address: arossi@pd.infn.it
} 


\section{Introduction}

In the last years the interest for the issue of neutrino mass generation has been renewed, especially by the SuperKamiokande data on atmospheric neutrinos [1]. These data can be explained by $\nu_{\mu}-\nu_{\tau}$ oscillation within the following parameter range (at $99 \% \mathrm{CL}$ ):

$$
\begin{aligned}
& \delta m_{\text {atm }}^{2}=(1-9) \times 10^{-3} \mathrm{eV}^{2}, \\
& \sin ^{2} 2 \theta_{\text {atm }}>0.8
\end{aligned}
$$

(the best-fit values are $\delta m_{\mathrm{atm}}^{2}=3 \times 10^{-3} \mathrm{eV}^{2}$ and $\sin ^{2} 2 \theta_{\mathrm{atm}}=0.99$ ), while the explanation through the $\nu_{\mu}-\nu_{e}$ oscillation is strongly disfavoured [2]. On the other hand, the solar neutrino data [3] can be interpreted in terms of $\nu_{e}$ oscillation into $\nu_{\mu}, \nu_{\tau}$, or their mixture within the following parameter range [四, 5]:

$$
\begin{aligned}
& \delta m_{\mathrm{sol}}^{2}=(3-10) \times 10^{-6} \mathrm{eV}^{2}, \\
& \sin ^{2} 2 \theta_{\mathrm{sol}}=(0.1-1.4) \cdot 10^{-2}
\end{aligned}
$$

(the best-fit values are $\delta m_{\text {sol }}^{2}=5 \times 10^{-6} \mathrm{eV}^{2}$ and $\sin ^{2} 2 \theta_{\text {sol }}=7 \times 10^{-3}$ ), the so called smallmixing angle MSW solution [6]. [] Therefore, the experimental data provide a strong evidence that like charged fermions, also neutrinos have masses and weak mixing. Namely, barring the less natural possibility that the neutrino mass eigenstates $\nu_{1,2,3}$ are strongly degenerate and assuming that $m_{3}>m_{2}>m_{1}$, the $\delta m^{2}$ ranges in eqs. (1) and (2) directly translate into

$$
\begin{aligned}
& m_{3} \simeq\left(\delta m_{\mathrm{atm}}^{2}\right)^{1 / 2}=(3-10) \cdot 10^{-2} \mathrm{eV}, \\
& m_{2} \simeq\left(\delta m_{\mathrm{sol}}^{2}\right)^{1 / 2}=(1.7-3.3) \cdot 10^{-3} \mathrm{eV},
\end{aligned}
$$

which indicates that the neutrino mass ratio $m_{3} / m_{2}$ is similar to that of charged fermions, $m_{\tau} / m_{\mu}$ or $m_{b} / m_{s}$. However, the neutrino mixing pattern inferred from (1) and (2) is drastically different from the well-established structure of the Cabibbo-Kobayashi-Maskawa (CKM) mixing of quarks.

The explanation of the fermion flavour structure is one of the most challenging problems of particle physics, and the neutrino case constitutes a part of this issue. In the standard model the charged fermion masses emerge from the Yukawa terms:t?

$$
u_{i}^{c} \mathbf{Y}_{u}^{i j} q_{j} H_{u}+d_{i}^{c} \mathbf{Y}_{d}^{i j} q_{j} H_{d}+e_{i}^{c} \mathbf{Y}_{e}^{i j} l_{j} H_{d},
$$

where $q_{i}=(u, d)_{i}, u_{i}^{c}, d_{i}^{c}$ and $l_{i}=(\nu, e)_{i}, e_{i}^{c}$ are the quark and lepton fields of three families $(i=1,2,3)$, and $H_{u, d}$ are the Higgs doublets, with the vacuum expectation values (VEVs) $v_{u, d}$ determining the electroweak scale, $\left(v_{u}^{2}+v_{d}^{2}\right)^{1 / 2}=v_{W}=174 \mathrm{GeV}$. There is no renormalizable term that gives rise to the neutrino masses. However, the latter can get Majorana masses from the lepton-number violating higher order operator

$$
\frac{1}{M_{L}} l_{i} \mathbf{Y}_{\nu}^{i j} l_{j} H_{u}^{2}, \quad \mathbf{Y}_{\nu}=\mathbf{Y}_{\nu}^{T}
$$

\footnotetext{
${ }^{3}$ We concentrate mainly on this solution, whose parameter range naturally emerges in the models presented below. One has to remark, however, that the solar neutrino data are still ambiguous and cannot discriminate other possible solutions. In particular, the recent analysis show that the large-mixing angle MSW and long wavelength "just-so" oscillation solutions have comparable statistical significance [2, 5.

${ }^{4}$ In the following we mainly deal with the supersymmetric model, though the general features of our discussion are equally valid for the non-supersymmetric case.
} 
which is cutoff by some large scale $M_{L}$, e.g. the grand unification or Planck scale [7].9 Hence, the charged fermion masses are $\sim v_{W}$ and the neutrino masses are $\sim v_{W}^{2} / M_{L}$ which makes it easy to understand why the latter are so light.

The quark mixing is originated from the misalignment between the Yukawa matrices $\mathbf{Y}_{u}$ and $\mathbf{Y}_{d}$. Analogously, the neutrino mixing is due to misalignment between $\mathbf{Y}_{e}$ and $\mathbf{Y}_{\nu}$. In particular, one can choose a basis where $\mathbf{Y}_{u}$ and $\mathbf{Y}_{\nu}$ are diagonal, $\mathbf{Y}_{u}=\operatorname{Diag}\left(Y_{u}, Y_{c}, Y_{t}\right)$ and $\mathbf{Y}_{\nu}=\operatorname{Diag}\left(Y_{1}, Y_{2}, Y_{3}\right)$. In this basis, the quark and lepton mixing angles are determined respectively by the form of $\mathbf{Y}_{d}$ and $\mathbf{Y}_{e}$ which in general remain non-diagonal. The latter are to be diagonalized by the bi-unitary transformations:

$$
\begin{aligned}
& U_{d}^{\prime T} \mathbf{Y}_{d} U_{d}=\mathbf{Y}_{d}^{D}=\operatorname{Diag}\left(Y_{d}, Y_{s}, Y_{b}\right) \\
& U_{e}^{\prime T} \mathbf{Y}_{e} U_{e}=\mathbf{Y}_{e}^{D}=\operatorname{Diag}\left(Y_{e}, Y_{\mu}, Y_{\tau}\right)
\end{aligned}
$$

with the unitary matrices $U_{d, e}$ rotating the left handed $(\mathrm{LH})$ states and with $U_{d, e}^{\prime}$ rotating the right handed $(\mathrm{RH})$ ones. Thus, in this basis the CKM matrix of weak mixing between the physical quark states $(u, c, t)$ and $(d, s, b)$ is $V_{q}=U_{d}$, while the matrix $V_{l}=U_{e}^{\dagger}$ determines the properties of the weak current between the charged leptons $(e, \mu, \tau)$ and the neutrinos. Namely, $V_{l}$ relates the neutrino flavour eigenstates $\left(\nu_{e}, \nu_{\mu}, \nu_{\tau}\right)$ to the mass eigenstates $\left(\nu_{1}, \nu_{2}, \nu_{3}\right)$, and describes the neutrino oscillation phenomena.

The unitary matrices

$$
V_{q}=\left(\begin{array}{ccc}
V_{u d} & V_{u s} & V_{u b} \\
V_{c d} & V_{c s} & V_{c b} \\
V_{t d} & V_{t s} & V_{t b}
\end{array}\right), \quad V_{l}=\left(\begin{array}{ccc}
V_{e 1} & V_{e 2} & V_{e 3} \\
V_{\mu 1} & V_{\mu 2} & V_{\mu 3} \\
V_{\tau 1} & V_{\tau 2} & V_{\tau 3}
\end{array}\right)
$$

are usually parameterised as [9]:

$$
V_{q, l}=\left(\begin{array}{ccc}
c_{12} c_{13} & s_{12} c_{13} & s_{13} e^{-i \delta} \\
-s_{12} c_{23}-c_{12} s_{23} s_{13} e^{i \delta} & c_{12} c_{23}-s_{12} s_{23} s_{13} e^{i \delta} & s_{23} c_{13} \\
s_{12} s_{23}-c_{12} c_{23} s_{13} e^{i \delta} & -c_{12} s_{23}-s_{12} c_{23} s_{13} e^{i \delta} & c_{23} c_{13}
\end{array}\right)_{q, l}
$$

where $s_{i j}\left(c_{i j}\right)$ stand for the sines (cosines) of the three mixing angles $\theta_{12}, \theta_{23}$ and $\theta_{13}$ while $\delta$ is the $\mathrm{CP}$ violating phase. (In the case of Majorana neutrinos, the lepton mixing matrix contains two additional phases $\delta_{1,2}$, factorised out as $V_{l} \cdot \operatorname{Diag}\left(1, e^{i \delta_{1}}, e^{i \delta_{2}}\right)$, which, however, are not relevant for neutrino oscillations.) In the following, we distinguish the angles and phases in $V_{q}$ and $V_{l}$ by the superscripts ' $q$ ' and ' $l$ ', respectively.

The explanation of the fermion mass and mixing pattern is beyond the capacities of the standard model. The Yukawa matrices $\mathbf{Y}_{u, d, e}$, are arbitrary complex $3 \times 3$ matrices, not constrained by any symmetry property. Concerning the neutrinos, apart from the Yukawa factors $\mathbf{Y}_{\nu}$ in (5), also the lepton-number violation scale $M_{L}$ is an arbitrary parameter. The magnitude of the latter can be inferred from the mass value $m_{3}$ in (3). Namely, from $m_{3} \sim$ $Y_{3} v_{W}^{2} / M_{L}$ we obtain

$$
Y_{3}^{-1} M_{L} \sim 10^{15} \mathrm{GeV}
$$

which is rather close to the GUT scale $M_{G} \sim 10^{16} \mathrm{GeV}$. In particular, the normalization of the operator (5) as $M_{L}=M_{G}$ implies $Y_{3} \sim 10$. Let us remark that there is no contradiction

\footnotetext{
${ }^{5}$ Any known mechanism for the neutrino masses reduces to such an effective operator. E.g., in the 'see-saw' scheme [8] it is obtained after integrating out heavy-neutral fermions with Majorana masses $\sim M_{L}$.
} 


\begin{tabular}{||l||l||}
\hline \hline \multicolumn{1}{||c||}{$2^{\text {nd }} / 1^{s t}$} & \multicolumn{1}{|c}{$3^{r d} / 2^{\text {nd }}$} \\
\hline \hline$Y_{c} / Y_{u}=250-1000$ & $Y_{t} / Y_{c}=230-330_{\left(\mu=M_{W}\right)}, 320-1000_{\left(\mu=M_{G}\right)}$ \\
\hline$Y_{s} / Y_{d}=17-25$ & $Y_{b} / Y_{s}=30-85_{\left(\mu=M_{W}\right)}, 33-120_{\left(\mu=M_{G}\right)}$ \\
\hline$Y_{\mu} / Y_{e}=207$ & $Y_{\tau} / Y_{\mu}=17$ \\
\hline$Y_{2} / Y_{1}>1$ & $Y_{3} / Y_{2}=10-60$ \\
\hline \hline
\end{tabular}

Table 1: Quark and lepton mass ratios between second/first and third/second families. All mass ratios are independent of the renormalization scale $\mu$ except $Y_{t} / Y_{c}$ and $Y_{b} / Y_{s}$ (in the case of reasonably small $\left.\tan \beta \equiv v_{u} / v_{d}\right)$. For definiteness, the latter ratios are evaluated at the electroweak scale, $\mu=M_{W}$, while at the GUT scale, $\mu=M_{G} \simeq 10^{16} \mathrm{GeV}$, they are scaled respectively as $B_{t}^{-3}$ and $B_{t}^{-1}$, with the factor $B_{t}=B\left(Y_{t}\right)$ varying from 0.9 to 0.7 for the top Yukawa constant at the GUT scale in the interval $Y_{t}^{G}=0.5-3$ (see details in section 4 ).

if the effective coupling constant $Y_{3}$ is large. E.g. in the context of the see-saw mechanism [8] $Y_{3}$ is determined by the ratio $Y_{\text {Dirac }}^{2} / Y_{\text {Majorana }}$ which can easily happen to be $\sim 10$ even if the Yukawa constants $Y_{\text {Dirac }}$ and $Y_{\text {Majorana }}$ are order 1.

One aspect of the flavour problem is related to the inter-family mass/Yukawa hierarchy among fermions [10]. For example, the top Yukawa constant is $Y_{t} \sim 1$, while the first-generation constants $Y_{e, u, d}$ are much smaller, $\sim 10^{-5}$. Concerning the neutrinos, they also seem to indicate a mass hierarchy similar to that of the charged fermions. In particular, eqs. (3) show that the ratio $Y_{3} / Y_{2}$ lies in the range $10-60$. There are no data restricting the ratio $Y_{2} / Y_{1}$, but it may also be large. For the sake of comparison, the mass ratios between different families are summarized in the Table 1 .

On the other hand, the mixing structure is very different between quarks and leptons (see Table 2). The most striking feature is that the atmospheric neutrino data favour the nearly maximal 23 mixing of neutrinos, while the 23 mixing of quarks is very small. On the contrary, the MSW solution implies a very small 12 mixing angle for neutrinos as compared to the sizeable 12 angle of the Cabibbo mixing for quarks.

It is widely believed that the fermion flavour structure can be understood within the context of the grand unified theories with horizontal symmetries. The latter acts between the fermion families and thus can help to constrain the form of the Yukawa matrices $\mathbf{Y}_{u, d, e, \nu}$. In particular, it can dictate the hierarchical and closely aligned structures of $\mathbf{Y}_{u}$ and $\mathbf{Y}_{d}$ and thus simultaneously explain the large mass splitting among different families as well as the smallness of the quark mixing angles. However, the origin of the large neutrino mixing is not a priori clear. Thus, the crucial point that must be explained by any realistic flavour theory is the dramatic difference between the quark and lepton mixing angles.

In a previous paper [13] we have outlined that such a complementary pattern of quark and lepton mixings can naturally emerge in the context of grand unified theories. In the $S U(5)$ model the quark and lepton states of each family are combined in the multiplets $\overline{5}_{i}=\left(d^{c}, l\right)_{i}$, $10_{i}=\left(u^{c}, e^{c}, q\right)_{i}$, and the Higgs doublets $H_{u}$ and $H_{d}$ fit respectively into the representations

\footnotetext{
${ }^{6}$ In last years many efforts have been applied to derive the neutrino mixing pattern from particular textures of the neutrino mass matrix [11]. Differently from some point of view adopted in [11], we believe that a satisfactory scheme can be obtained in the context of a well-defined and complete flavour theory including the charged fermions. Such attempts have been taken e.g. in refs. [12 where the neutrino mixing pattern was discussed in the context of grand unified and horizontal symmetries.
} 


\begin{tabular}{||c|c|c||}
\hline \hline & $q$ (uark) & $l($ epton $)$ \\
\hline$\theta_{12}$ & $12.7 \pm 0.1$ & $2.4 \pm 1.0$ \\
\hline$\theta_{23}$ & $2.3 \pm 0.1$ & $45 \pm 13$ \\
\hline$\theta_{13}$ & $0.21 \pm 0.03$ & $<15$ \\
\hline \hline
\end{tabular}

Table 2: Quark and leptonic mixing angles (in ${ }^{\circ}$ ). The values of $\theta_{23}^{l}$ and $\theta_{12}^{l}$ correspond to the $99 \% \mathrm{CL}$ regions of the AN and SN data fitting, eqs. (11) and (2), while the upper bound for $\theta_{13}^{l}$ comes from the combination of CHOOZ [14] and atmospheric neutrino data [2]. The values of $\theta_{12}^{q}, \theta_{23}^{q}$ and $\theta_{13}^{q}$ reflect the present data on $\left|V_{u s}\right|,\left|V_{c b}\right|$ and $\left|V_{u b} / V_{c b}\right|$ [9]. The last two values depend on the renormalization scale and for $\mu=M_{G}$ they should be scaled by a factor $B_{t}$, i.e. become smaller by $(10-30) \%$.

$H \sim 5$ and $\bar{H} \sim \overline{5}$. The terms responsible for the fermion masses read as:

$$
10_{i} \mathbf{G}_{u}^{i j} 10_{j} H+\overline{5}_{i} \mathbf{G}^{i j} 10_{j} \bar{H}+\frac{1}{M_{L}} \overline{5}_{i} \mathbf{G}_{\nu}^{i j} \overline{5}_{j} H^{2},
$$

where the Yukawa constant matrices $\mathbf{G}_{u}$ and $\mathbf{G}_{\nu}$ are symmetric due to $S U(5)$ symmetry reasons while the form of $\mathbf{G}$ is not constrained. After the $S U(5)$ symmetry breaking these terms reduce to the standard model terms (41) and (5), with

$$
\begin{aligned}
\mathbf{Y}_{u}=\mathbf{G}_{u}, & \mathbf{Y}_{\nu}=\mathbf{G}_{\nu}, \\
\mathbf{Y}_{d}=\mathbf{G}, & \mathbf{Y}_{e}=\mathbf{G}^{T} .
\end{aligned}
$$

Hence, in the basis where the matrices $\mathbf{G}_{u}$ and $\mathbf{G}_{\nu}$ are diagonal, the rotation angles of the LH leptons coincide with that of the RH quarks: $U_{e}=U_{d}^{\prime}$, and vice versa, $U_{d}=U_{e}^{\prime}$. (One has to remark, that in grand unification the rotation angles of $\mathrm{RH}$ states also have a physical sense as they define the fermion mixing in B- and L-violating currents.) Hence, if $G^{23} \sim G^{33}$ while $G^{32} \ll G^{33}$, the large 23 neutrino mixing will occur together with the small 23 mixing of quarks [12, 13]. In other words, the phenomenology requires that the matrix $G$ should have a strongly asymmetric form.

In particular, a very interesting situation emerges in the case of Fritzsch-like texture 15 with vanishing $G^{22}$ and $G^{11}$ entries. In addition, the entry $G^{33}$ can be taken as $S U(5)$ singlet thus maintaining the $b-\tau$ Yukawa unification, while the off-diagonal entries $G^{23}$ etc. should contain some $S U(5)$ Clebsches to avoid the wrong relations $Y_{d, s}=Y_{e, \mu}$ of the minimal $S U(5)$. This means that the masses of the third generation emerge from the Yukawa couplings to the Higgs 5-plet, while the lighter generation masses are contributed also by 45-plet. In fact, it is not necessary to introduce the elementary Higgs 45-plet. Instead, the off-diagonal entries $\mathbf{G}^{i j}$ can be regarded as operators dependent on the adjoint Higgs $\Phi \sim 24$ of $S U(5), \mathbf{G}^{i j}=\mathbf{G}^{i j}(\Phi)$. In this case the relation $\mathbf{Y}_{d}=\mathbf{Y}_{e}^{T}$ is not exact anymore, but it fulfills with the precision of these Clebsch factors. Nevertheless, this property ensures that between the 23 mixing angles

\footnotetext{
7 This should be understood as expansion series $\mathbf{G}^{i j}(\Phi)=G^{(0) i j}+G^{(1) i j} \frac{\Phi}{M}+\ldots$, where $M$ is some cutoff mass larger than the GUT scale (e.g. the string scale). This means that the off-diagonal terms emerge from the effective higher-order operators $\frac{\Phi}{M} \bar{H} \overline{5}_{i} G^{(1) i j} 10_{j}$ etc., just on the same footing as the last term in (10). Since in general the operator $\Phi \cdot \bar{H}$ is represented by the tensor product $24 \times \overline{5}=\overline{5}+\overline{45}$, it can distinguish the corresponding entries in the matrices $\mathbf{Y}_{e}$ and $\mathbf{Y}_{d}$ and hence avoid the lepton-quark degeneracy of the minimal $S U(5)$ theory.
} 
of quarks and leptons the following relation is fulfilled with a good accuracy [13]:

$$
\tan \theta_{23}^{q} \tan \theta_{23}^{l} \simeq\left(\frac{m_{\mu} m_{s}}{m_{\tau} m_{b}}\right)^{1 / 2}
$$

This product rule indeed works remarkably well. It demonstrates a 'see-saw' correspondence between the lepton and quark mixing angles and tells us that whenever the neutrino mixing is large, $\tan \theta_{23}^{l} \sim 1$, the quark mixing angle comes out small and in the correct range, $\tan \theta_{23}^{q} \sim$ 0.04 .

Namely, in ref. [13] we have considered the following Yukawa pattern:

$$
\begin{aligned}
\mathbf{Y}_{u}=\left(\begin{array}{ccc}
Y_{u} & 0 & 0 \\
0 & Y_{c} & 0 \\
0 & 0 & Y_{t}
\end{array}\right), & \mathbf{Y}_{d}=\left(\begin{array}{ccc}
0 & A_{d} & 0 \\
A_{d}^{\prime} & 0 & B_{d} \\
0 & B_{d}^{\prime} & D
\end{array}\right)=\left(\begin{array}{ccc}
0 & A_{d} & 0 \\
\frac{1}{a} A_{d} & 0 & B_{d} \\
0 & \frac{1}{b} B_{d} & D
\end{array}\right), \\
\mathbf{Y}_{\nu}=\left(\begin{array}{ccc}
Y_{1} & 0 & 0 \\
0 & Y_{2} & 0 \\
0 & 0 & Y_{3}
\end{array}\right), & \mathbf{Y}_{e}^{T}=\left(\begin{array}{ccc}
0 & A_{e} & 0 \\
A_{e}^{\prime} & 0 & B_{e} \\
0 & B_{e}^{\prime} & D
\end{array}\right)=\left(\begin{array}{ccc}
0 & A_{e} & 0 \\
\frac{1}{a} A_{e} & 0 & B_{e} \\
0 & \frac{1}{b} B_{e} & D
\end{array}\right),
\end{aligned}
$$

where the parameters $b=B_{e} / B_{e}^{\prime}=B_{d} / B_{d}^{\prime}$ and $a=A_{e} / A_{e}^{\prime}=A_{d} / A_{d}^{\prime}$ reflect the possible asymmetry between the off-diagonal entries in $\mathbf{Y}_{d, e}$. In addition, the Clebsches $A_{e} / A_{d}=k_{A}$ and $B_{e} / B_{d}=k_{B}$ are allowed to be different from 1 and their values can be extracted from the quark and lepton masses.

Indeed, the texture predicts the following relations between the Yukawa eigenvalues [13]:

$$
\frac{Y_{b}}{Y_{\tau}}=Z, \quad \frac{Y_{s}-Y_{d}}{Y_{\mu}-Y_{e}}=\frac{1}{k_{B}^{2} Z}, \quad \frac{Y_{d} Y_{s}}{Y_{e} Y_{\mu}}=\frac{1}{k_{A}^{2} Z}
$$

and

$$
\frac{m_{s}}{m_{d}}+\frac{m_{d}}{m_{s}}-2=\frac{k_{A}^{2}}{Z k_{B}^{4}}\left(\frac{m_{\mu}}{m_{e}}+\frac{m_{e}}{m_{\mu}}-2\right),
$$

where

$$
Z=\sqrt{1-\left(b+b^{-1}\right)\left(1-k_{B}^{-2}\right) \frac{m_{\mu}-m_{e}}{m_{\tau}}} .
$$

In particular, the phenomenologically correct picture for the fermion masses emerges if the asymmetry parameter $b$ is large enough, $b \sim 10$, and when $k_{A} \simeq 1$ and $k_{B} \simeq 2$ [13]. In this case the relation (15) leads to the correct prediction for the strange/down quark mass ratio.

On the other hand, for the 23 mixing angles in (7) we obtain the relations:

$$
\tan \theta_{23}^{l} \simeq b^{1 / 2} \sqrt{\frac{m_{\mu}}{m_{\tau}}}, \quad \tan \theta_{23}^{q} \simeq b^{-1 / 2} \sqrt{\frac{m_{s}}{m_{b}}},
$$

from which the product rule (12) is immediate. These relations also point to a large asymmetry factor $b$. In particular, for $b \sim 10$ the neutrino mixing becomes nearly maximal, $\theta_{23}^{l} \sim 45^{\circ}$, whereas the quark mixing angle gets small, $\theta_{23}^{q} \sim(2-3)^{\circ}$.

\footnotetext{
${ }^{8}$ The symmetric case $b=1$, i.e. $\mathbf{Y}_{d, e}$ having the familiar Fritzsch texture, is obviously excluded, since the 23 mixing of quarks is too big, $\theta_{23}^{q}=(6-10)^{\circ}$, while it is too small for leptons, $\theta_{23}^{l}=13.7^{\circ}$ (cfr. the experimental values in Table 2].
} 
In the general case, for arbitrary $a$, the product rule similar to that in (17) can be obtained also for 12 mixing angles. However, from phenomenological grounds one can deduce that no significant asymmetry should occur in the 12 sector of the matrices (13). Namely, the successful relation for the Cabibbo angle:

$$
\tan \theta_{12}^{q} \simeq \sqrt{\frac{m_{d}}{m_{s}}}
$$

indicates that $A_{d} \simeq A_{d}^{\prime}$, so that $a=1$ is appropriate. Interestingly, in this case we obtain for the leptonic 12 mixing [13]:

$$
\tan \theta_{12}^{l} \simeq \sqrt{\cos \theta_{23}^{l} \frac{m_{e}}{m_{\mu}}}
$$

Given that $\theta_{23}^{l} \sim 45^{\circ}$, this implies $\theta_{12}^{l} \sim 3^{\circ}$, which is in the range relevant to the MSW solution of the SN problem (cfr. Table 2).

Therefore, for $a=1$ the ansatz (13) depends on six parameters, so it is highly predictive. Among these, three Yukawa entries $A_{e}, B_{e}, D$ can be expressed in terms of lepton couplings $Y_{e, \mu, \tau}$ and $b$. Thus 9 physical quantities, the Yukawa eigenvalues $Y_{d, s, b}$ and the mixing angles in $V_{q}$ and $V_{l}$, are left as functions of three Clebsch factors, $k_{B}, k_{A}$ and $b$. Hence, at the GUT scale these are connected by six relations like (14), (17), etc. As discussed above, in the first approximation these relations well reproduce the observed pattern of the quark and lepton mixing angles for $b \sim 10$ and $k_{B} \simeq 2, k_{A} \simeq 1$. However, a closer inspection shows that for precise Clebsch values $k_{B}=2$ and $k_{A}=1$, which could be motivated in the GUT context, and $a=1$ which could follow from the $U(2)_{H}$ horizontal symmetry, the predictions for $\left|V_{u s}\right|$ and $\left|V_{c b}\right|$ substantially deviate from the corresponding experimental values which presently are known with a very good accuracy. The case summarized above implies a real CKM matrix since $\mathbf{Y}_{u}$ is taken diagonal and so all phases in $\mathbf{Y}_{d}$ can be absorbed by the field re-definitions. Nevertheless, it would be interesting to address the CP issue in a scenario in which $\mathbf{Y}_{u}$ is still kept diagonal.

In the present paper we suggest a new grand unified ansatz for the fermion masses, which is an extension of the pattern (13) of ref. [13. Namely, we consider the case when the matrices $\mathbf{G}_{u}$ and $\mathbf{G}_{\nu}$ are both diagonal and related as $\mathbf{G}_{\nu} \propto \mathbf{b}^{\prime-1} \cdot \mathbf{G}_{u}$, with $\mathbf{b}^{\prime}$ being a diagonal matrix $\mathbf{b}^{\prime}=\operatorname{Diag}\left(1,1, b^{\prime}\right)$. Regarding $\mathbf{G}$, we assume that it has the diagonal part $\propto \mathbf{G}_{u}$ and the off-diagonal one $\propto \mathbf{b}^{-1} \cdot \mathbf{A}(\Phi)$, where $\mathbf{A}$ is an antisymmetric matrix with $\Phi$-field dependent entries inducing different Clebsch factors for the down quarks and charged leptons, and $\mathbf{b}=\operatorname{Diag}(1,1, b)$. In other words, we consider the Yukawa textures:

$$
\begin{gathered}
\mathbf{Y}_{u}=\mathbf{Y}_{u}^{D}, \quad \mathbf{Y}_{d}=\rho \mathbf{Y}_{u}+\mathbf{b}^{-1} \mathbf{A}_{d} \\
\mathbf{Y}_{\nu}=\eta \mathbf{b}^{\prime-1} \mathbf{Y}_{u},
\end{gathered}
$$

where $\rho$ and $\eta$ are proportionality coefficients. For $b=1$ this pattern resembles the so-called Stech texture proposed long time ago by Stech [16] and independently by Chkareuli and one of the authors [17], where the off-diagonal entries of $\mathbf{Y}_{d}$ were assumed to be antisymmetric, $\mathbf{Y}_{d}=\rho \mathbf{Y}_{u}+\mathbf{A}_{d}$. This case, however, is completely excluded by experimental data. As for our texture, the matrices $\mathbf{b}^{-1} \mathbf{A}_{d, e}$ remain antisymmetric only in the 12 block, while the other entries are skew due to the factor $b \neq 1$. In the explicit form the Yukawa matrices read as:

$$
\mathbf{Y}_{u}=\left(\begin{array}{ccc}
Y_{u} & 0 & 0 \\
0 & Y_{c} & 0 \\
0 & 0 & Y_{t}
\end{array}\right), \quad \mathbf{Y}_{d}=\left(\begin{array}{ccc}
\frac{Y_{u}}{Y_{t}} D & A_{d} & C_{d} \\
-A_{d} & \frac{Y_{c}}{Y_{t}} D & B_{d} \\
-\frac{1}{b} C_{d} & -\frac{1}{b} B_{d} & D
\end{array}\right)
$$




$$
\mathbf{Y}_{\nu}=\eta\left(\begin{array}{ccc}
Y_{u} & 0 & 0 \\
0 & Y_{c} & 0 \\
0 & 0 & \frac{1}{b^{\prime}} Y_{t}
\end{array}\right), \quad \mathbf{Y}_{e}^{T}=\left(\begin{array}{ccc}
\frac{Y_{u}}{Y_{t}} D & A_{e} & C_{e} \\
-A_{e} & \frac{Y_{c}}{Y_{t}} D & B_{e} \\
-\frac{1}{b} C_{e} & -\frac{1}{b} B_{e} & D
\end{array}\right)
$$

Clearly this texture represents an extension of the Fritzsch-like 'zero-texture' (13) considered in [13. In particular, for vanishing 13 and 31 entries, $C_{d, e}=0$, this texture essentially reduces to the latter as the diagonal 11 and 22 entries in $\mathbf{Y}_{d, e}(20)$ are quite small, since the ratios $Y_{u} / Y_{t}$ and $Y_{c} / Y_{t}$ are much smaller than $1 / 3$ and $2 / 3$ Yukawa ratios in the down quark and charged leptons. In practice, as we show below, one can safely take the limit $Y_{u} / Y_{t} \rightarrow 0$ and ignore the 11 entries in $\mathbf{Y}_{d, e}$. As for the 22 entry, though it is small, it can provide significant corrections (in particular, to $V_{c b}$ ). In principle, it also contains the relative (un-removable) CP violating phase which however cannot provide a sufficient amount of $\mathrm{CP}$ violation. This can be remedied by non-vanishing $C_{e, d}$.

Moreover, we show that for large values of $b(\sim 10)$, the texture (21) provides a very appealing description of the quark and lepton mass and mixing structures. Interestingly, the case $b^{\prime} \sim b \sim 10$ also provides nice relations between neutrino and up-quark masses, $Y_{2} / Y_{3}=b^{\prime} Y_{c} / Y_{t}$ (cfr. Table 1$)$.

Hence, the complete pattern (21) is first motivated on the phenomenological point of view as it can fit the physical observables with excellent precision. From a theoretical point of view, we show that these textures can be justified in the framework of grand unification by invoking also the concept of horizontal symmetry among three fermion families [18, 17].

The paper is organized as follows. In Sect. 2 we discuss some theoretical tools for buildingup the textures (20) in a realistic and predictive manner in the framework of supersymmetric $S U(5)$ model with $S U(3)_{H}$ horizontal symmetry. The discussion in Sect. 3 is meant to provide an existence proof of those realizations in the framework of $S O(10) \times S U(3)_{H}$ model. Three particular ansätze are singled out, characterized by different assignments of the Clebsch factors between the quark and lepton entries, and in Sect. 4 their phenomenological analysis is presented and discussed in all details. Finally, we summarise our findings and conclude.

\section{Theoretical framework: GUT and horizontal symmetry}

In this section we would like to sketch the general ideas needed to obtain the Yukawa textures (20) in the $S U(5)$ model with the $S U(3)_{H}$ horizontal symmetry [18, 17. In the course of the presentation, it will become apparent that the discussion has to take a less general character as for example some additional symmetries should be invoked. This aspect will be faced in Sect. 2.2. In the discussion $S U(5)$ is taken as a prototype theory featuring the basic properties of grand unification. In a more general context one could think of GUTs based on larger groups (e.g. $S U(N)$ with $N \geq 6$ ) which contain $S U(5)$ and perhaps also unify both $S U(5)$ and $S U(3)_{H}$. A particular model based on $S O(10) \times S U(3)_{H}$ will be presented in the next section.

\subsection{General aspects}

We consider the supersymmetric $S U(5)$ model with the horizontal symmetry $S U(3)_{H}$ where three fermion families are unified in chiral superfields:

$$
\overline{5}_{i}=\left(d^{c}, l\right)_{i} \sim(\overline{5}, 3), \quad 10_{i}=\left(u^{c}, q, e^{c}\right)_{i} \sim(10,3),
$$


$\left(i=1,2,3\right.$ is $S U(3)_{H}$ index), while the Higgs superfields $5, \overline{5}$ are singlets of $S U(3)_{H}$ :

$$
H \sim(5,1), \quad \bar{H} \sim(\overline{5}, 1) .
$$

We also assume that the theory is invariant under R-parity. In other words, we impose the matter parity $Z_{2}$ under which the matter superfields change the sign while the Higgs ones are invariant. ']

Since the fermion bilinears transform as $3 \times 3=\overline{3}+6$, their "standard" Yukawa couplings to the Higgses are forbidden by the horizontal symmetry. Hence, the fermion masses can be induced only by higher order operators involving a set of "horizontal" Higgs superfields $X^{i j}$ in two-index representations of $S U(3)_{H}$ : symmetric $X_{s}^{i j} \equiv S^{\{i j\}} \sim(1, \overline{6})$ and antisymmetric $X_{a}^{i j} \equiv A^{[i j]}=\varepsilon^{i j k} A_{k} \sim(1,3):$ 田

$$
\mathcal{O}_{u}=\frac{S^{i j}}{M} 10_{i} 10_{j} H, \quad \mathcal{O}=\frac{S^{i j}+A^{i j}}{M} \overline{5}_{i} 10_{j} \bar{H}, \quad \mathcal{O}_{\nu}=\frac{S^{i j}}{M^{2}} \overline{5}_{i} \overline{5}_{j} H^{2}
$$

where $M$ is some large scale (the flavour scale). Needless to say, by $S U(5)$ symmetry reasons, the antisymmetric Higgses $A$ can participate only in $\mathcal{O}$. Therefore, in the low-energy limit the operators (24) reduce to the Yukawa couplings:

$$
\mathbf{Y}_{u} \sim \frac{\langle S\rangle}{M}, \quad \mathbf{Y}_{d}, \mathbf{Y}_{e}^{T} \sim \frac{\langle S\rangle+\langle A\rangle}{M}, \quad \frac{\mathbf{Y}_{\nu}}{M_{L}} \sim \frac{\langle S\rangle}{M^{2}}
$$

In this way, the fermion mass hierarchy can be naturally linked to the hierarchy of the horizontal-symmetry breaking scales [17, 20]. For more details on the horizontal VEV structures, see e.g. [21].

In particular, let us assume that the horizontal Higgses include one sextet $S$ with a VEV taken diagonal:

$$
\left\langle S^{i j}\right\rangle=\left(\begin{array}{ccc}
\mathcal{S}_{1} & 0 & 0 \\
0 & \mathcal{S}_{2} & 0 \\
0 & 0 & \mathcal{S}_{3}
\end{array}\right), \quad \mathcal{S}_{3} \gg \mathcal{S}_{2} \gg \mathcal{S}_{1},
$$

and a set of triplets $A_{n}^{i j} \equiv \varepsilon^{i j k} A_{n k}, n=1,2,3$, which in general have the VEVs towards all three components:

$$
\sum_{n}\left\langle A_{n}^{i j}\right\rangle=\left(\begin{array}{ccc}
0 & \mathcal{A}_{3} & \mathcal{A}_{2} \\
-\mathcal{A}_{3} & 0 & \mathcal{A}_{1} \\
-\mathcal{A}_{2} & -\mathcal{A}_{1} & 0
\end{array}\right), \quad \mathcal{A}_{1}>\mathcal{A}_{2}>\mathcal{A}_{3} .
$$

(see Appendix A). Then from (25) we see that $Y_{t} \sim 1$ implies $\mathcal{S}_{3} \sim M$ which indeed can naturally arise from the Higgs sector as we show in Appendix A. Similarly one can expect

\footnotetext{
${ }^{9}$ The $S U(3)_{H}$ symmetry itself does not not prevent the $R$-parity violating couplings $\varepsilon^{i j k} 10_{i} \overline{5}_{j} \overline{5}_{k}$ from where the $B-L$ violating terms $u^{c} d^{c} d^{c}, q d^{c} l, e^{c} l l$ arise. For a general discussion on the relation between $R$ parity and horizontal symmetries, see [19]. In particular, in case of the horizontal symmetry $S U(4)_{H}$ the R-parity could emerge as an accidental symmetry. One has to remark, however, that in many cases considered below the matter parity automatically follows from the discrete symmetries imposed on the model.

10 The following discussion can equally apply in both the cases of global and local horizontal symmetry, though it would be more appealing to regard $S U(3)_{H}$ as gauge symmetry, like $S U(5)$.

${ }^{11}$ The theory may also contain conjugated Higgses $\bar{X}_{i j}$ in representations $\bar{S} \sim(1,6)$ and $\bar{A} \sim(1, \overline{3})$. These usually are needed for writing non-trivial superpotential terms in order to generate the horizontal VEVs (see Appendix A). The conjugated fields, however, do not couple the fermion superfields (22) and thus do not contribute to the quark and lepton masses.
} 
that also $Y_{b, \tau} \sim 1$ which would require large $\tan \beta$ regime. However, as we shall see below, in realistic schemes also small $\tan \beta$ can be naturally accommodated. In addition, the operator $\mathcal{O}_{\nu}$ translates into $Y_{3} / M_{L} \sim \mathcal{S}_{3} / M^{2} \sim Y_{t} / M$. Therefore, the flavour scale $M$ is of the order of the B-L violating scale $M_{L} \sim 10^{15} \mathrm{GeV}$ (cfr. (9)). The closeness of the flavour scale with the GUT scale $M_{G} \sim 10^{16} \mathrm{GeV}$ suggests that they may have a common origin. (The mismatch between the estimate of $M$ and $M_{G}$ can be due to some spread of the coupling constants in the theory.)

It is well known that the effective operators (24) may arise entirely from a renormalizable theory, after integrating out some heavy degrees of freedom [22], and thereby the flavour scale can be related to the mass scale of the latter. It is also plausible to think that this mass scale is set by large (order GUT scale) VEV of some Higgs $\Omega,\langle\Omega\rangle \sim M$.

In particular, one can introduce vector-like states in the 10 - and $\overline{10}$-representations of $S U(5)$ :

$$
T^{i}=\left(U^{c}, Q, E^{c}\right)^{i} \sim(10, \overline{3}), \quad \bar{T}_{i}=\left(U, Q^{c}, E\right)_{i} \sim(\overline{10}, 3),
$$

and the $S U(5)$-singlet fields

$$
N^{i} \sim(1, \overline{3}), \quad \bar{N}_{i} \sim(1,3),
$$

and consider the following Yukawa couplings in the superpotential:

$$
\begin{aligned}
& W_{T}=10 H T+\overline{5} \bar{H} T+\bar{T} X 10+T \Omega \bar{T} \\
& W_{N}=\overline{5} H N+X_{s} \bar{N}^{2}+N \Omega \bar{N}
\end{aligned}
$$

(family indices are suppressed, and order 1 coupling constants are understood in each term). Clearly, in $W_{T}$ the horizontal Higgs $X$ can be both of the type $X_{s}=S$ or $X_{a}=A$, whereas in $W_{N}$ only the symmetric Higgses $X_{s}$ can participate. Since $T, \bar{T}$ contain heavy states with electric charges $2 / 3,-1 / 3$ and -1 , their exchange can induce the operators $\mathcal{O}_{u}$ and $\mathcal{O}$ relevant for the masses of all charged fermions: up quarks, down quarks and leptons. Analogously, the operator $\mathcal{O}_{\nu}$ for the neutrino masses emerges via the exchange of the singlet states $N, \bar{N}$. The relevant diagrams are shown in Fig. 1.

Therefore, after integrating out the heavy states, we obtain the operators (24) in the form

$$
\begin{aligned}
& \mathcal{O}_{u}=10\left(H \mathbf{M}_{T}^{-1} X+X^{T} \mathbf{M}_{T}^{-1} H\right) 10=2 \cdot 10 H\left(\mathbf{M}_{T}^{-1} S\right) 10+10 H\left(\mathbf{M}_{T}^{-1} A-A \mathbf{M}_{T}^{-1}\right) 10 \\
& \mathcal{O}=\overline{5}\left(\bar{H} \mathbf{M}_{T}^{-1} X\right) 10=\overline{5} \bar{H}\left(\mathbf{M}_{T}^{-1} S+\mathbf{M}_{T}^{-1} A\right) 10 \\
& \mathcal{O}_{\nu}=\overline{5} H \mathbf{M}_{N}^{-1} S \mathbf{M}_{N}^{-1} H \overline{5}
\end{aligned}
$$

where $\mathbf{M}_{T}, \mathbf{M}_{N}$ are $3 \times 3$ mass matrices of the heavy states, induced from their couplings to the Higgs $\Omega$. Therefore, if $\Omega$ is a gauge singlet, then $\mathbf{M}_{T}, \mathbf{M}_{N}$ will be degenerate in family space, $\mathbf{M}_{T, N} \sim M \cdot \mathbf{1}$, where $\mathbf{1}=\operatorname{Diag}(1,1,1)$ is the unit matrix of $S U(3)_{H}$. In this case, if $X$ are $S U(5)$ singlets, only the symmetric fields $X_{s}=X_{s}^{T}$ can contribute $\mathcal{O}_{u}$ in (31), while the contributions of the antisymmetric ones $X_{a}=-X_{a}^{T}$ cancel out. On the other hand, both $X_{s}$ and $X_{a}$ can contribute to $\mathcal{O}$. Therefore, the operators (31) reduce to the Yukawa couplings of the form:

$$
\mathbf{Y}_{u} \sim \mathbf{M}_{T}^{-1}\langle S\rangle, \quad \mathbf{Y}_{d}, \mathbf{Y}_{e}^{T} \sim \mathbf{M}_{T}^{-1}\langle S\rangle+\mathbf{M}_{T}^{-1}\langle A\rangle, \quad M_{L}^{-1} \mathbf{Y}_{\nu} \sim \mathbf{M}_{N}^{-2}\langle S\rangle
$$

More explicitly, these can be written as:

$$
\mathbf{Y}_{u} \propto \mathbf{S}, \quad \mathbf{Y}_{\nu} \propto \mathbf{S}, \quad \mathbf{Y}_{d}=\mathbf{Y}_{e}^{T} \propto \mathbf{S}+\mathbf{A},
$$




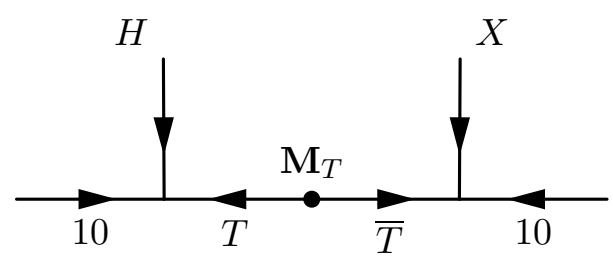

(a)

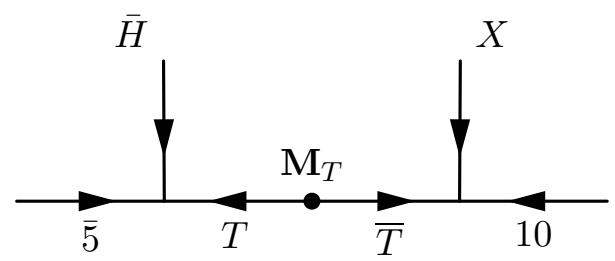

(b)

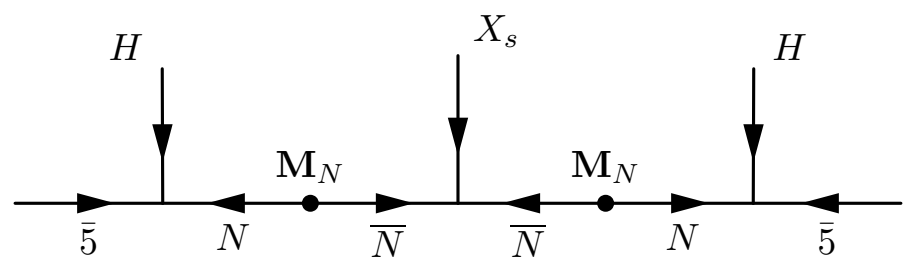

(c)

Figure 1: The heavy fermion exchanges giving rise to the effective higher order operators for the fermion masses: (a) for up quarks, (b) for down quarks and charged leptons, (c) for neutrinos.

where $\mathbf{S}=M^{-1}\langle S\rangle$ and $\mathbf{A}=M^{-1}\langle A\rangle$ are respectively the symmetric and antisymmetric matrices reflecting the form of the VEVs (26) and (27). (We omit the dimensionless factors which keep track of the coupling constants in (30) and are not specified at the moment).

It should be immediately noted, however, that the texture (33) is far from being realistic for many reasons:

(A) $\mathbf{Y}_{d} \propto \mathbf{Y}_{u}+\mathbf{A}$ with antisymmetric $\mathbf{A}$ leads to very big 23 mixing, $s_{23}^{q} \simeq\left(Y_{s} / Y_{b}\right)^{1 / 2} \sim$ $0.1-0.2$, much above the experimental value of $V_{c b}$.

(B) the relation $\mathbf{Y}_{\nu} \propto \mathbf{Y}_{u}$ implies $Y_{1}: Y_{2}: Y_{3}=Y_{u}: Y_{c}: Y_{t}$ and thus too big a hierarchy between the neutrino mass eigenvalues $m_{3}$ and $m_{2}$ (cfr. Table 1).

(C) finally, $\mathbf{Y}_{d}=\mathbf{Y}_{e}^{T}$ features the minimal $S U(5)$ degeneracy of the Yukawa couplings, $Y_{d, s, b}=$ $Y_{e, \mu, \tau}$, whose drawbacks have been already recalled.

The latter problem can be easily cured by making use of the adjoint superfield $\Phi$ which breaks $S U(5)$ down to $S U(3) \times S U(2) \times U(1)$ by the VEV proportional to the hypercharge generator: $\langle\Phi\rangle \propto \lambda_{y} \equiv \operatorname{Diag}(1 / 3,1 / 3,1 / 3,-1 / 2,-1 / 2)$. Thus if the matrix $A$ in (25) effectively contains $\Phi, A=A(\Phi)$, effective Clebsch factors emerge discriminating quarks from leptons. Therefore, at least some of the antisymmetric Higgses $A$ can be taken in the mixed representation $A \sim(24,3)$. 12 The tensor product is $\overline{5} \times 24=\overline{5}+\overline{45}+70$, where only the first two terms are relevant for the fermion bilinear $\overline{5} \times 10=5+45$. Then the unwanted relations $Y_{d, s}=Y_{e, \mu}$ can be avoided while maintaining the successful relation $Y_{b} \simeq Y_{\tau}$.

The first two problems can be solved in a similar way, by making use of the adjoint Higgs of $S U(3)_{H}$. The masses of the heavy fermions transform as $3 \times \overline{3}=1+8$ representations. Hence one can assume that the set of Higgses $\Omega$ contains, besides the singlet $I \sim(1,1)$, also

\footnotetext{
12 Alternatively, one can take all the antisymmetric Higgses as $S U(5)$ singlets, $A \sim(1,3)$, but assume that some of them act in combination with $\Phi \sim(24,1)$, to end up with the effective operator $\frac{A \Phi}{M^{2}} \overline{5} \cdot 10 \bar{H}(\mathrm{cfr}$. (24)) so that the tensor product $\bar{H} \cdot \Phi \cdot A$ effectively contains both $(\overline{5}, 3)$ and $(\overline{45}, 3)$ representations. Such an attitude will be taken in constructing the $S O(10)$ model in Sect. 3 .
} 
the superfield $\Sigma \sim(1,8)$ with the $\operatorname{VEV}\langle\Sigma\rangle$ pointing towards the $\lambda_{8}$ generator: $[3$

$$
\langle\Sigma\rangle \sim M \cdot\left(\begin{array}{ccc}
1 & 0 & 0 \\
0 & 1 & 0 \\
0 & 0 & -2
\end{array}\right) .
$$

This VEV breaks $S U(3)_{H}$ down to $S U(2)_{H} \times U(1)_{H}$ and thus discriminates the third fermion family from the others [23]. Hence, the heavy-fermion mass matrices $\mathbf{M}_{T, N}$ maintain only the $S U(2)_{H}$ invariant form $\mathbf{M}=M \cdot \operatorname{Diag}(1,1, b)$, with $b$ in general different from 1 . In this way, from (32) we can obtain the desired Yukawa pattern (20). [4]

Notice however, that for achieving our goal, we have implemented the adjoint structures ( $\Sigma$ into $M$ and $\Phi$ into $A$ ) in eq. (32) which is not the right place. Indeed, (32) follows from (31) if and only if neither $M$ nor $A$ contain adjoint representations of $S U(5)$ or $S U(3)_{H}$. For example, if $A$ contains the $S U(5)$ adjoint, then the tensor product $A \times H \sim(24,3) \times(5,1)$ effectively contains the $(45,3)$ representation which would induce off-diagonal antisymmetric contribution also to $\mathbf{Y}_{u}$. On the other hand, if $A$ 's are $S U(5)$ singlet, but $\mathbf{M}_{T}$ contains the $S U(3)_{H}$ octet, then $(1,3) \times(1,8) \times(5,1)$ effectively contains $(5, \overline{6})$ which would still give rise to symmetric off-diagonal entries in $\mathbf{Y}_{u}$. Therefore, in either case the antisymmetric VEVs $\langle A\rangle$ contribute the first term in (24) and so induce off-diagonal entries in $\mathbf{Y}_{u}$ ruining in such a way the desired pattern. Tackling this issue requires more theoretical ingredients and tools which will be discussed below.

\subsection{Some specific realizations}

Thus, we have seen that in order to arrive at the desired textures (20), both the $S U(3)_{H}$ singlet $I \sim(1,1)$ and octet $\Sigma \sim(1,8)$ are needed for generating the heavy fermion masses. In addition, the diagrams contributing via the $S$ and $A$ type Higgses are to be "differentiated" by some additional symmetry so that $S$ will contribute to all the matrices $\mathbf{Y}_{u}, \mathbf{Y}_{\nu}$ and $\mathbf{Y}_{d, e}$, while $A$ 's only to $\mathbf{Y}_{d, e}$. Hence, we have to assume that besides the local $S U(5) \times S U(3)_{H}$ symmetry, the theory is invariant under some set $\mathcal{G}$ of abelian symmetries which may contain e.g. non-anomalous or pseudo-anomalous gauge $U(1)$ factors, continuous or discrete R-symmetry, discrete symmetries like $Z_{2}, Z_{3}$, etc.

First, on the basis of the considerations outlined in the previous section, we put forward the needed couplings in the superpotential. Second, those will be motivated by some additional symmetry of $\mathcal{G}$.

Now about the first step. We assume that the flavour scale is determined by the VEVs of the singlet $I \sim(1,1)$ and the octet $\Sigma \sim(1,8)$, respectively $\langle I\rangle=M$ and $\langle\Sigma\rangle=x M \cdot \operatorname{Diag}(1,1,-2)$, $x \sim 1$. Then the combination $I+\Sigma$ provides order $M$ masses to the heavy fermions, splitting the third generation from the first two and maintaining the latter degenerate. As in the previous subsection, we take the horizontal VEVs in the form (26) and (27) and define the dimensionless VEV matrices as $\mathbf{S} \equiv M^{-1}\langle S\rangle$ and $\mathbf{A}_{n} \equiv M^{-1}\left\langle A_{n}\right\rangle$.

\footnotetext{
${ }^{13}$ In the following of the paper, the symbol $\Omega$ stands for a set of Higgs fields in real representations of the gauge groups. When needed the content of $\Omega$ will be specified as singlet $I \sim(1,1)$, octet $\Sigma \sim(1,8)$ or 24 -plet $\Phi \sim(24,1)$.

14 In group-theoretical language this can be rephrased as follows. The tensor product $A \cdot \Sigma$ reads as $3 \times 8=$ $3+\overline{6}+15$ where both 3 and $\overline{6}$ can match the fermion bilinears $3 \times 3$. In this way, the off-diagonal entries in $\mathbf{Y}_{e, d}$ are not anymore antisymmetric - only the 12 sector keeps on the antisymmetry owing to the residual $S U(2)_{H}$ symmetry.
} 
- We further assume that the superpotential $W_{T}(30)$ related to heavy 10-plets (28) involves some set of horizontal Higgses $X$, which in fact consists of the sextet $S \sim(1, \overline{6})$. Therefore, $W_{T}$ becomes:

$$
W_{T}=f 10 H T+g \overline{5} \bar{H} T+\lambda_{T} \bar{T} X 10+T\left(\alpha_{T} I+\beta_{T} \Sigma\right) \bar{T}
$$

where we explicitly indicate the order 1 coupling constants. So the diagonal entries in $\mathbf{Y}_{u}$ and $\mathbf{Y}_{d, e}$ are induced respectively via the diagrams (a,b) of Fig. 1. In order to respect the $b-\tau$ Yukawa unification, the 24-plet $\Phi \sim(24,1)$ should not couple to $T \bar{T}$. 1:

- The couplings in $W_{N}$ are left as in (30) and they induce $\mathbf{Y}_{\nu}$ via the diagram (c) in Fig.

$$
W_{N}=h \overline{5} H N+\lambda_{N} S \bar{N}^{2}+N\left(\alpha_{N} I+\beta_{N} \Sigma\right) \bar{N} .
$$

Thus, $\mathbf{Y}_{\nu}$ is diagonal in the VEV basis (26).

- Let us consider another set of horizontal Higgses $X^{\prime}$, which contains antisymmetric fields $A_{n}$ in the representations $(1,3)$ and $(24,3)$, and assume that they contribute only to $\mathbf{Y}_{d, e}$ via the exchange of other heavy fermions. The simplest possibility is to introduce additional states in 5- and $\overline{5}$-representations of $S U(5)$ :

$$
\bar{F}^{i}=\left(D^{c}, L\right)^{i} \sim(\overline{5}, \overline{3}), \quad F_{i}=\left(D, L^{c}\right)_{i} \sim(5,3)
$$

having the following couplings in the superpotential:

$$
W_{F}=\lambda_{F} \overline{5} X^{\prime} F+g^{\prime} \bar{F} \bar{H}^{\prime} 10+F\left(\alpha_{F} I+\beta_{F} \Sigma\right) \bar{F},
$$

where $\bar{H}^{\prime}$ is another Higgs $\overline{5}$-plet (or $\overline{45}$-plet) of $S U(5)$. The corresponding diagram is shown in Fig. 2(a). In the following, such a scenario will be called $F$-scheme.

Alternatively, instead of the $F+\bar{F}$ one can involve an additional pair of 10-plets

$$
T^{i}=\left(U^{\prime c}, Q^{\prime}, E^{\prime c}\right)^{i} \sim(10, \overline{3}), \quad \bar{T}_{i}^{\prime}=\left(U^{\prime}, Q^{\prime c}, E^{\prime}\right)_{i} \sim(\overline{10}, 3)
$$

with the superpotential terms:

$$
W_{T^{\prime}}=g^{\prime} \overline{5} \bar{H}^{\prime} T^{\prime}+\lambda_{T}^{\prime} \bar{T}^{\prime} X^{\prime} 10+T^{\prime}\left(\alpha_{T}^{\prime} I+\beta_{T}^{\prime} \Sigma\right) \bar{T}^{\prime} .
$$

The corresponding exchange is shown in Fig. 2(b). This scenario will be referred to as the $T^{\prime}$-scheme.

In either scheme, the standard Higgs doublet $H_{d}$ should be regarded a superposition of the doublet components in $\bar{H}$ and $\bar{H}^{\prime}: H_{d}=c_{\omega} \bar{H}_{2}^{\prime}-s_{\omega} \bar{H}_{2}$, which is rendered light after arranging the doublet-triplet splitting, while the orthogonal combination $s_{\omega} \bar{H}_{2}^{\prime}+c_{\omega} \bar{H}_{2}$ is left heavy, with mass $\sim M_{G}$ (here $\omega$ is a mixing angle and $\left.c_{\omega}\left(s_{\omega}\right)=\cos \omega(\sin \omega)\right)$. On the other hand, we assume that $H_{u}$ comes entirely from $H$, while the field $H^{\prime}$, though present, is just a spectator in the Yukawa sector. On the contrary, $H_{d}$ is contained in both $\bar{H}$ and $\bar{H}^{\prime}$ with weights $s_{\omega}$ and $c_{\omega}$ respectively.

Once the fields $A_{n}=\left(X_{a}\right)_{n}$ contain the mixed representation $(24,3)$ and/or $\bar{H}^{\prime}$ is 45 -plet, the diagrams in Fig. 2 2 induce off-diagonal entries in $\mathbf{Y}_{d}$ and $\mathbf{Y}_{e}^{T}$ with different Clebsch factors.

Let us consider in some more details e.g. the $F$-scheme. After substituting the VEVs of the horizontal Higgses, the couplings (35), (36) and (38) give rise to the following field-dependent 


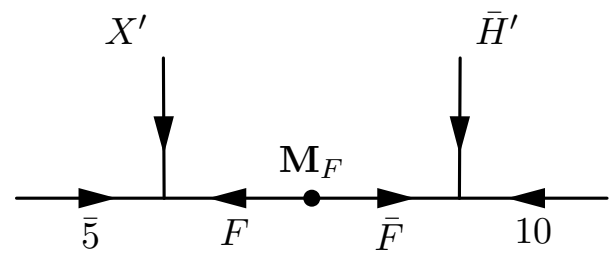

(a)

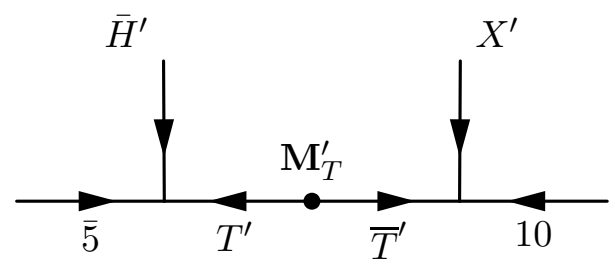

(b)

Figure 2: The diagrams inducing the off-diagonal contributions to $\mathbf{Y}_{d, e}$ : (a) exchange of 5-plets (Fscheme), (b) exchange of additional 10-plets ( $T^{\prime}$-scheme).

mass matrices for the up quark and neutrino states:

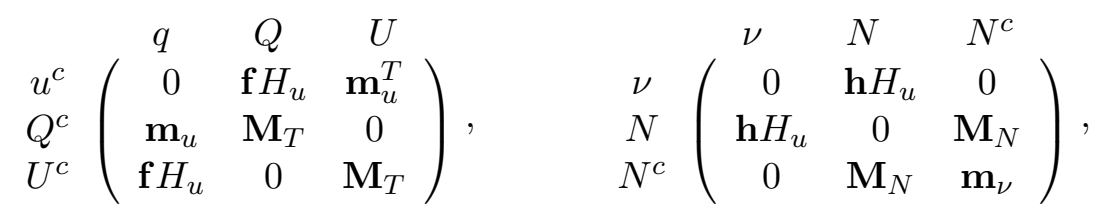

and for the down quark and charged lepton states:

$$
\left.\begin{array}{l}
d^{c} \\
Q^{c} \\
D^{c}
\end{array}\left(\begin{array}{ccc}
q & Q & D \\
0 & \mathbf{g} \bar{H}_{2} & \mathbf{m}_{d} \\
\mathbf{m}_{u} & \mathbf{M}_{T} & 0 \\
\mathbf{g}^{\prime} \eta_{d} \bar{H}_{2}^{\prime} & 0 & \mathbf{M}_{F}
\end{array}\right), \quad \begin{array}{l}
e^{c} \\
E^{c}
\end{array} \quad \begin{array}{ccc}
l & E & L \\
0 & \mathbf{m}_{u}^{T} & \mathbf{g}^{\prime} \eta_{e} \bar{H}_{2}^{\prime} \\
\mathbf{g} \bar{H}_{2} & \mathbf{M}_{T} & 0 \\
\mathbf{m}_{l}^{T} & 0 & \mathbf{M}_{F}
\end{array}\right),
$$

where $\eta_{d, e}$ are the Clebsch factors dependent on the $S U(5)$ content of $\bar{H}^{\prime}$. Namely, $\eta_{e}=\eta_{d}$ if $\bar{H}^{\prime}$ is $\overline{5}$-plet and $\eta_{e}=-3 \eta_{d}$ if $\bar{H}^{\prime}$ is $\overline{45}$-plet. In the above, each entry is a $3 \times 3$ matrix in the $S U(3)_{H}$ space. In particular $\mathbf{f}=f \mathbf{1}, \mathbf{h}=h \mathbf{1}$, etc. are flavour-blind $\left(S U(3)_{H}\right.$ degenerate) matrices.

As long as the heavy fermion mass matrices are induced by $I$ and $\Sigma$, they will be diagonal and have $S U(2)_{H}$ invariant shape $\mathbf{M}_{\Psi}=a_{\Psi} M \mathbf{b}_{\psi}(\Psi=T, F, N)$, where

$$
\mathbf{b}_{\Psi}=\left(\begin{array}{ccc}
1 & 0 & 0 \\
0 & 1 & 0 \\
0 & 0 & b_{\Psi}
\end{array}\right), \quad a_{\psi}=\alpha_{\Psi}+x \beta_{\Psi}, \quad b_{\psi}=\frac{\alpha_{\Psi}-2 x \beta_{\Psi}}{\alpha_{\Psi}+x \beta_{\Psi}}
$$

These will give rise to the deformation factors $\mathbf{b}$ and $\mathbf{b}^{\prime}$ in (20) whose relations with the factors $\mathbf{b}_{\psi}$ are easy to catch - at any rate they will be shown later.

Thus, the main information on the fermion flavour pattern is contained in the matrices $\mathbf{m}_{u, d, l, \nu}$. The matrices $\mathbf{m}_{u, \nu}$ are proportional to the $\operatorname{VEV}\langle S\rangle=\operatorname{Diag}\left(\mathcal{S}_{1}, \mathcal{S}_{2}, \mathcal{S}_{3}\right), \mathbf{m}_{u}=\lambda_{T} M \mathbf{S}$ and $\mathbf{m}_{\nu}=\lambda_{N} M \mathbf{S}$. As for the matrices $\mathbf{m}_{d, e}$, they are antisymmetric and emerge from the VEVs of the triplets $A_{n}$ sketched in eq. (27). As far as the latter set contain $(1,3)$ and $(24,3)$ representations, all those entries in general are non-trivial $5 \times 5$ matrices, $\tilde{\mathcal{A}}_{k}=\mathcal{A}_{k}\left(1+\zeta_{k} \lambda_{y}\right)$, where $\mathbb{1}$ and $\lambda_{y}$ are the unit and hypercharge matrices in $S U(5)$ space, respectively and $\zeta_{k}$ measures the relative weight of the singlet and 24-plet VEVs at each component $(k=1,2,3)$. Thus, the corresponding entries in $\mathbf{m}_{d}$ and $\mathbf{m}_{l}^{T}$ differ by Clebsch factors $C_{k}^{d, e}=1+\zeta_{k} y_{d, l}$, where $y_{d}=1 / 3$ and $y_{l}=-1 / 2$ are the hypercharges of the $d^{c}$ and $l$ fragments of $\overline{5}$-plets. 
Therefore, after integrating out the heavy states, we obtain an effective low energy theory with the following Yukawa matrices:

$$
\begin{aligned}
& \mathbf{Y}_{u}=f\left(\mathbf{M}_{T}^{-1} \mathbf{m}_{u}+\mathbf{m}_{u}^{T} \mathbf{M}_{T}^{-1}\right) \\
& \mathbf{Y}_{d}=g s_{\omega} \mathbf{M}_{T}^{-1} \mathbf{m}_{u}+g^{\prime} c_{\omega} \eta_{d} \mathbf{m}_{d} \mathbf{M}_{F}^{-1} \\
& \mathbf{Y}_{e}^{T}=g s_{\omega} \mathbf{M}_{T}^{-1} \mathbf{m}_{u}+g^{\prime} c_{\omega} \eta_{e} \mathbf{m}_{l} \mathbf{M}_{F}^{-1}
\end{aligned}
$$

and

$$
\frac{\mathbf{Y}_{\nu}}{M_{L}}=h^{2} \mathbf{M}_{N}^{-2} \mathbf{m}_{\nu}
$$

Therefore, $\mathbf{Y}_{u}$ and $\mathbf{Y}_{\nu}$ get contribution only from $S$ :

$$
\mathbf{Y}_{u}=\frac{2 f \lambda_{T}}{a_{T}} \mathbf{b}_{T}^{-1} \mathbf{S}, \quad \frac{\mathbf{Y}_{\nu}}{M_{L}}=\frac{h^{2} \lambda_{N}}{a_{N}^{2} M} \mathbf{b}_{N}^{-2} \mathbf{S}
$$

and thus both have diagonal forms related as:

$$
\frac{\mathbf{Y}_{\nu}}{M_{L}}=\eta \mathbf{b}^{\prime-1} \frac{\mathbf{Y}_{u}}{M}
$$

where $\mathbf{b}^{\prime}=\operatorname{Diag}\left(1,1, b^{\prime}\right)$, and

$$
b^{\prime}=\frac{b_{N}^{2}}{b_{T}}, \quad \eta=\frac{h^{2} \lambda_{N} a_{T}}{2 f \lambda_{T} a_{N}^{2}} .
$$

As for $\mathbf{Y}_{e, d}$, they have a diagonal contribution $g s_{\omega} /(2 f) \mathbf{Y}_{u}$ from the first term in (44), and off-diagonal entries $\mathbf{A}_{n} \mathbf{b}_{F}^{-1}$ from the the second term. Therefore, the full matrices $\mathbf{Y}_{d, e}$ can be rewritten as

$$
\mathbf{Y}_{d}=\rho \mathbf{Y}_{u}+\mathbf{b}^{-1} \mathbf{A}_{d}, \quad \mathbf{Y}_{e}^{T}=\rho \mathbf{Y}_{u}+\mathbf{b}^{-1} \mathbf{A}_{e}
$$

where $\rho=\frac{g}{2 f} s_{\omega}$ and $\mathbf{b}=\operatorname{Diag}(1,1, b)$, with $b=b_{F}^{-1}$, while the other dimensionless coefficients, such as the Clebsch factors related to the representations of $\bar{H}^{\prime}$ and $A$, are absorbed into the definition of the entries of the antisymmetric matrices

$$
\mathbf{A}_{d, e}=\left(\begin{array}{ccc}
0 & A_{3}^{d, e} & A_{2}^{d, e} \\
-A_{3}^{d, e} & 0 & A_{1}^{d, e} \\
-A_{2}^{d, e} & -A_{1}^{d, e} & 0
\end{array}\right)
$$

so that the relative Clebsch factors are defined as $k_{i}=A_{i}^{e} / A_{i}^{d}=\frac{\eta_{e} C_{i}^{e}}{\eta_{d} C_{i}^{d}}$. Putting everything together, we can write the Yukawa matrices as:

$$
\begin{array}{ccc}
\mathbf{Y}_{u}=\left(\begin{array}{ccc}
Y_{u} & 0 & 0 \\
0 & Y_{c} & 0 \\
0 & 0 & Y_{t}
\end{array}\right), & \mathbf{Y}_{d}=\left(\begin{array}{ccc}
\rho Y_{u} & A_{3}^{d} & A_{2}^{d} \\
-A_{3}^{d} & \rho Y_{c} & A_{1}^{d} \\
-\frac{1}{b} A_{2}^{d} & -\frac{1}{b} A_{1}^{d} & \rho Y_{t}
\end{array}\right), \\
\mathbf{Y}_{\nu}=\eta\left(\begin{array}{ccc}
Y_{u} & 0 & 0 \\
0 & Y_{c} & 0 \\
0 & 0 & \frac{1}{b^{\prime}} Y_{t}
\end{array}\right), & \mathbf{Y}_{e}^{T}=\left(\begin{array}{ccc}
\rho Y_{u} & A_{3}^{e} & A_{2}^{e} \\
-A_{3}^{e} & \rho Y_{c} & A_{1}^{e} \\
-\frac{1}{b} A_{2}^{e} & -\frac{1}{b} A_{1}^{e} & \rho Y_{t}
\end{array}\right) .
\end{array}
$$

The following comments are in order. 
- First of all, $Y_{t} \sim 1$ implies that $\mathcal{S}_{3} \sim b_{T} M$, while the relations $Y_{u}: Y_{c}: Y_{t} \sim \varepsilon^{2}: \varepsilon: b_{T}^{-1}$, well fits the upper quark mass hierarchy for $\varepsilon \sim 10^{-2}$ and $b_{T} \sim 0.1-1$. As already noted in Sect. 2.1 this situation can naturally emerge from the pattern of the horizontal Higgs superpotential (see Appendix A).

- Since the $(3,3)$ element in $\mathbf{Y}_{d, e}$ is also related to largest scale $\mathcal{S}_{3}$, we have approximate $b-\tau$ Yukawa unification at the GUT scale: $Y_{\tau} \simeq Y_{b} \simeq \rho Y_{t}$. On the other hand, as in principle the mixing angle $\omega$ between the doublets in $\bar{H}$ and $\bar{H}^{\prime}$ can be small, i.e. $\sin \omega<1$, the case $Y_{b} / Y_{t} \ll 1$ is plausible even if the constants $f$ and $g$ are both of order 1 . Therefore, the regime of moderate $\tan \beta$ is quite a natural possibility, in which case the renormalization group equations can be substantially simplified. In the following we assume that regime, though the case with large $\tan \beta$ should not be excluded.

- The large 23 lepton mixing implies $A_{1}^{e} \sim Y_{\tau}$. This also seems natural in the context of the model as the largest triplet VEV $\mathcal{A}_{1}$ should be of the order of the flavour scale scale $M$ (see Appendix A). On the other hand, the small 23 mixing of quarks needs $A_{1}^{d} / b<0.1 Y_{b}$, i.e. a large asymmetry parameter, $b \sim 10$, which in turn implies $b_{F} \sim 0.1$. In this case we observe an inverse hierarchy between the first two and third family of the heavy $F$ states, i.e. $M_{1,2}^{F}>M_{3}^{F}$.

- Concerning the neutrino Yukawa eigenvalues, they obey the relations

$$
\frac{Y_{1}}{Y_{2}}=\frac{Y_{u}}{Y_{c}} \ll 1, \quad \frac{Y_{2}}{Y_{3}}=b^{\prime} \frac{Y_{c}}{Y_{t}}
$$

which also points to large $b^{\prime}$ (cfr. Table 1), $b^{\prime} \sim 5-100$. In particular, the relation $b^{\prime}=b$ could work, which can occur if $b_{T}=b_{F}$ and $b_{N}=1$ - that is when the same singlet-octet combination $I+\Sigma$ acts in $W_{T}$ and $W_{F}$, while in $W_{N}$ only the singlet $I$ is present $\left(\beta_{N}=0\right.$ in (36)).

- On the other hand, we obtain (cfr. (9))

$$
\frac{M_{L}}{Y_{3}}=\eta^{-1} b^{\prime} \frac{M}{Y_{t}} \sim 10^{15} \mathrm{GeV}
$$

Since $Y_{t} \sim 1$ and $b^{\prime} \sim 10$, this translates into $\eta \sim 10^{2}$ if $M \sim 10^{16} \mathrm{GeV}$, i.e. order GUT scale. It should not be, however, very surprising to find $\eta \sim 10^{2}$ due to some spread of the coupling constants in the theory (cfr. (48)).

Let us remark that the set $X$ appearing in eq. (35) could contain also a triplet Higgs $A_{3} \sim(1,3)$ having a VEV towards the third component, $\left\langle A_{3}^{i j}\right\rangle=\varepsilon^{i j 3} \mathcal{A}_{3}$. This would induce antisymmetric 12 entries in the matrix $\mathbf{m}_{u}$. This, however, would not alter the diagonal form of $\mathbf{Y}_{u}$, since in the combination $\mathbf{M}_{T}^{-1} \mathbf{m}_{u}+\mathbf{m}_{u}^{T} \mathbf{M}_{T}^{-1}$ the antisymmetric entries cancel out (thanks to the residual $S U(2)_{H}$ symmetry in $\mathbf{M}_{T}$ ). However, in $\mathbf{Y}_{d, e}$ this will induce antisymmetric 12 entries with Clebsch factors $k_{e}=k_{d}$.

The $T^{\prime}$-scheme can be developed along the same lines. The only difference will be that in this case we have $b=b_{T^{\prime}}$, so that there should be a direct hierarchy between the first two and third family of the heavy $T^{\prime}$ states, i.e. $M_{1,2}^{T^{\prime}}<M_{3}^{T^{\prime}}$. In this case $b^{\prime}=b$ can be obtained if $b_{T^{\prime}}=b_{N}=b_{T}$, i.e. when $W_{T, T^{\prime}, N}$ share the same $I+\Sigma$ combination. Alternatively, when $b_{T^{\prime}}=b_{N}$ but $b_{T}=1$ (in $W_{T}$ contributes only the singlet $I$ ), we would obtain another interesting possibility $b^{\prime}=b^{2}$ which can be also compatible with the experimental data. Namely, it would point towards smaller $3 / 2$ hierarchy between the neutrino mass eigenvalues: $Y_{3} / Y_{2} \sim 10$ (cfr. Table 1). 
We now come to the second step to prove that the superpotentials (35), (36) and (38) can be justified by some extra symmetry $\mathcal{G}$. First of all, we assume that $\mathcal{G}$ contains a discrete symmetry $\mathcal{R}$ under which all superfields in the theory as well as the superpotential change sign. This will allow only trilinear terms in the superpotential (provided that they are consistent also with $S U(5) \times S U(3)_{H}$ and other symmetries in $\mathcal{G}$ ), while some singlet $Y$ can have also linear term $\Lambda^{2} Y$. In this situation all VEVs (including the GUT and horizontal ones) can emerge from a single mass scale $\Lambda$.

Second, we introduce a $Z_{3}$ symmetry acting on the superfields as $\psi \rightarrow \psi \exp \left(\frac{2 \pi}{3} i Q_{\psi}\right)$, with the following 'charges' $Q_{\psi}$ :

$$
\begin{aligned}
Q=0: & Y, \Phi, \bar{H}^{\prime} ; T, \\
Q=1: & I, \Sigma, \bar{S}, A_{n}, H, H^{\prime} ; \overline{5}, F, \bar{F}, N \\
Q=-1: & \bar{I}, S, \bar{A}_{n}, \bar{H} ; 10, \bar{T}, \bar{N} .
\end{aligned}
$$

where as agreed $\Phi \sim(24,1), \Sigma \sim(1,8)$ and $I, \bar{I}$ and $Y$ are some singlets, $\sim(1,1)$,

The most general superpotential invariant under $\mathcal{R} \times Z_{3}$ is restricted to have the following form:

$$
\begin{aligned}
W_{\mathrm{Higgs}}= & \Lambda^{2} Y+Y^{3}+Y \Phi^{2}+\Phi^{3}+Y I \bar{I}+I^{3}+\bar{I}^{3}+I \Sigma^{2}+\Sigma^{3}+ \\
& Y\left(S \bar{S}+A_{n} \bar{A}_{n}\right)+S^{3}+\bar{S}^{3}+A_{1} A_{2} A_{3}+\bar{A}_{1} \bar{A}_{2} \bar{A}_{3}+ \\
& H(Y+\Phi) \bar{H}+H^{\prime}(Y+\Phi) \bar{H}+H^{\prime} I \bar{H}^{\prime}
\end{aligned}
$$

and

$$
\begin{aligned}
W_{\text {Yuk }}=\quad & 10\left(H+H^{\prime}\right) T+\overline{5} \bar{H} T+T(I+\Sigma) \bar{T}+\bar{T} S 10+ \\
& \overline{5} A F+F(I+\Sigma) \bar{F}+\bar{F} \bar{H}^{\prime} 10+ \\
& \overline{5} H N+N Y \bar{N}+S \bar{N}^{2}
\end{aligned}
$$

respectively in the Higgs and Yukawa sectors.

It is worth observing the following features of the Higgs potential. It has only one mass scale (in the linear term) which determines the physical scales present in the theory (GUT, flavour, etc.). Notice that among other degenerate minima, this superpotential allows a solution when all singlets, adjoints and sextet/triplets get VEVs linked to the scale $\Lambda$, modulo unspecified coupling constants in (55). For example, the superpotential terms for $S$ and $\bar{S}$ considered in Appendix A, emerge from here with $\mu \sim\langle Y\rangle$.

There are no couplings between $S, A$ and $\Sigma$, so the superpotential does not fix the relative orientation of their VEVs. However, that will be fixed by supersymmetry breaking terms, along the lines discussed in the Appendix A. For example, these can be D-terms like $\frac{1}{M^{2}} \int d \theta^{4} z \bar{z}\left[\sigma S^{\dagger} \Sigma^{\dagger} \Sigma S+\lambda_{n} A_{n}^{\dagger} \Sigma^{\dagger} \Sigma A_{n}\right]$ etc., where $z=m \theta^{2}$ stands for supersymmetry breaking spurion. Depending on the form and sign of the coupling constants, these terms can fix the relative VEV orientation of $\Sigma, S$ and $A_{n}$ in the $S U(3)_{H}$ space. In particular, the choice $\lambda_{1,2}>0$ favours "orthogonal" VEV directions of $A_{1,2}$ and $\Sigma$, while $\sigma<0$ prefers the "parallel" VEV directions of $S$ and $\Sigma$. Thus, in this case the largest entry in $S$ is positioned at the same place as in $\Sigma$. The same for other fields. A typical consequence of the fact that the horizontal VEV configuration is dynamically fixed by small terms, is the presence of light horizontal Pseudo-Goldstone Higgses in the theory, reminiscent of the familons, even if the horizontal 
symmetry is local [23]. As for the $S U(5)$ part, the adjoint $\Phi$ gets a VEV $\sim \Lambda$ and, as far as the superpotential contains also $\Phi^{3}$ term, no light fragments of $\Phi$ are left behind.

Notice that the term $H I \bar{H}^{\prime}$ is not present in (55) although it seems to be allowed by the symmetry. The point is that if $H^{\prime}$ is a 5 -plet, then as it has the same charges as $H$, that term can be rotated away by redefinition of $H$ and $H^{\prime}$. Instead, if $H^{\prime}$ and $\bar{H}^{\prime}$ are 45-plets, then that term is forbidden by the $S U(5)$ symmetry. Thus, after substituting the VEVs, the bilinears of the doublet and triplet fragments in $H, H^{\prime}$ and $\bar{H}, \bar{H}^{\prime}$ have the form:

$$
\left(\begin{array}{cc}
\lambda_{1} Y+\lambda_{2} \Phi & 0 \\
\lambda_{3} Y+\lambda_{4} \Phi & I
\end{array}\right),
$$

where $\lambda_{1}, \lambda_{2}, \cdots$ are $\mathcal{O}(1)$ coupling constants previously understood. For rendering the Higgses $H_{u, d}$ light, the 11 element of (57) in the corresponding doublet sector has to be fine tuned by canceling big contributions between $\lambda_{1} Y$ and $\lambda_{2} \Phi$. (Then the triplet components will remain heavy.) Therefore, $H_{u}$ is contained entirely in $H$ whereas $H_{d}$ emerges as a superposition of the doublet fragments in $\bar{H}$ and $\bar{H}^{\prime}, H_{d}=-s_{\omega} \bar{H}_{2}+c_{\omega} \bar{H}_{2}^{\prime}$, with $\tan \omega \sim \frac{\langle I\rangle}{\left\langle\lambda_{3} Y+\lambda_{4} \Phi\right\rangle}$, while the other combination of these doublets is superheavy.

The Yukawa superpotential (56) contains all terms of eqs. (35), (36) and (38) which lead to the Yukawa pattern (49). Furthermore, some specific features have arisen. The heavy-states $\bar{N}$ mass matrix emerges only by the VEV of singlet $Y$, so $b_{N}=1$ and thus $b^{\prime}=b_{T}^{-1}$. Then, if $I+\Sigma$ acts in the same reducible combination in $W_{T}$ and $W_{F}$, i.e. $b_{T}=b_{F}$ then $\mathbf{b}^{\prime}=\mathbf{b}$, which is phenomenologically welcome (52). For example, this could be the case if the reducible combination $I+\Sigma$ emerges from an adjoint Higgs of a larger group containing $S U(3)_{H}$, e.g. $S U(N+3)_{H}$. Following similar lines of reasoning, also the $T^{\prime}$ scheme can be justified.

Finally, in either schemes, the Clebsch coefficients $k_{n}=A_{n}^{e} / A_{n}^{d}, n=1,2,3$, depend on the $S U(5)$ content of the tensor products $A_{n} \cdot \bar{H}^{\prime}$. Needless to say that whatever values of the Clebsch factors can occur when the Higgses $A_{n}$ are in $\sim(1+24,3)$ with unspecified weights $\zeta_{n}$ of 1 and 24 component. However, it would be more attractive (though it as a matter of taste) to find a more "familiar" origin of the Clebsch factors phenomenologically needed. In other words, we assume (on similar grounds as e.g. in refs. 24]) that different entries in the Yukawa matrices are differentiated by some "clean" Clebsch factor $k_{n}$ in which the VEV structure of the original GUT is encoded through a specific representation of the intermediate heavy fermions (e.g. $F$ or $T^{\prime}$ in our case). Therefore, let us think that at each entry, the Clebsches $k_{n}$ can have some specific rational values such as $0, \pm \frac{1}{3}, \pm \frac{2}{3}, \pm 1, \pm \frac{3}{2}, \pm 2, \pm 3$, etc. , possibly related to various charges (electric, hypercharge, $B-L$ etc.).

In particular, in the context of $S U(5)$, whenever the product $A_{n} \cdot \bar{H}^{\prime}$ is in $\overline{5}$ - or $\overline{45}$-channel (e.g. simply when $A$ is singlet, $A \sim(1,3)$, and the Higgs $\bar{H}^{\prime}$ is in $\overline{5}$ or $\overline{45}$ representations), we have $k_{n}=1$ and $k_{n}=-3$, respectively. In Table 3 we report the values for $k_{n}$ attainable for $A \sim(1,3)$ and $A \sim(24,3)$ (or $M \sim 24$ ), too.

As in the next we shall consider in detail specific Clebsch patterns for the Yukawa matrices, characterized by different values of $k_{n}$, now we would like to keep the discussion on the Clebsch factors more general to show how we arrive at those specific choices.

Indeed, we could think of all these different ansätze in terms of larger vertical and/or horizontal symmetries. Namely, in these cases $S U(5)$ may emerge just a subgroup of some larger GUT symmetry group. (The $S O(10)$ example will be considered in the next section). Then the adjoint representation would generally emerge accompanied by a singlet partner. 


\begin{tabular}{||c||c|c|c|c||}
\hline \hline & $S U(5)$ rep. & $\begin{array}{c}A \sim 1, \\
M \sim 1\end{array}$ & $\begin{array}{c}A \sim 24, \\
M \sim 1\end{array}$ & $\begin{array}{c}A \sim 1, \\
M \sim 24\end{array}$ \\
\hline F - scheme & $H^{\prime} \sim \overline{5}$ & 1 & $-3 / 2$ & $-2 / 3$ \\
& $H^{\prime} \sim \overline{45}$ & -3 & $9 / 2$ & 2 \\
\hline$T^{\prime}$ - scheme & $H^{\prime} \sim \overline{5}$ & 1 & 6 & $1 / 6$ \\
& $H^{\prime} \sim \overline{45}$ & -3 & -18 & $-1 / 2$ \\
\hline \hline
\end{tabular}

Table 3: Possible values for the Clebsch factors $k_{n}=A_{n}^{e} / A_{n}^{d}$, as they can emerge in the $F$ - and $T^{\prime}$-versions for various assignments of the $S U(5)$ representations to the Higgses $\bar{H}^{\prime}$ and $A$ and to the heavy-state masses $M$. The case when both $A, M \sim 24$ gives the same pattern as $A, M \sim 1$. Clearly, in each case the Clebsches obtained for $\bar{H}^{\prime} \sim \overline{5}$ and $\bar{H}^{\prime} \sim \overline{45}$ differ just by a factor -3 .

Hence, the corresponding combination $1+24$ can have a VEV with two different eigenvalues

$$
\left\langle\Phi_{1+24}\right\rangle=\operatorname{Diag}(x, x, x, y, y)
$$

In the context of $F$-scheme (exchange of heavy 5-plets) this would produce a Clebsch $k=\frac{y}{x}$. In the case of 10-plet exchange ( $T^{\prime}$ scheme) the Clebsch value would be $k=\frac{2 y}{x+y}$.

For example, in the context of the $S U(6)$ model, which, as a matter of fact, provides a natural doublet-triplet splitting, the adjoint Higgs (35-plet) of $S U(6)$ plays a central role [25]. This 35 -plet $(35=1+24+5+\overline{5}$, in terms of $S U(5)$ subgroup) has a VEV $\sim \operatorname{Diag}(1,1,1,1,-2,-2)$. Therefore, in the context of $F$-like scheme it can provide a Clebsch factor $k=-2$, or, in $T^{\prime}$-like scheme, $k=4$ [25].

In fact, one can consider more general cases of $S U(N)$ group. Such a theory could contain adjoint Higgses with different structures which break $S U(N)$ in various possible channels. By imposing the traceless condition on the $S U(N)$ adjoint VEV, it is straightforward to obtain the relation between the two eigenvalues $x$ and $y$ in (58) and thus the relative Clebsch factors projected out in the context of the $F$-like and $T^{\prime}$-like schemes.

I. $\Phi_{1}: S U(N) \rightarrow S U(5) \times S U(N-5) \times U(1)$, this Higgs contains only the $S U(5)$ singlet VEV and thus can produce only $k=1$.

II. $\Phi_{2}: S U(N) \rightarrow S U(N-2) \times S U(2)_{w} \times U(1)$, displaying the weak isospin $S U(2)_{w}$. Hence $\left\langle\Phi_{2}\right\rangle=\operatorname{Diag} \cdot\left(1,1, \ldots,-\frac{N-2}{2},-\frac{N-2}{2}\right)$ which implemented in the $F$-like scheme, generates the Clebsches $k=-\frac{N-2}{2}$, i.e. $k=-2,-5 / 2,-3,-7 / 2, \cdots$ respectively for $N=6,7,8,9, \cdots$. In the context of $T^{\prime}$-like schemes the same VEV provides $k=\frac{2(N-2)}{N-4}$, i.e. $k=4,10 / 3,3,14 / 5, \cdots$.

III. $\Phi_{3}: S U(N) \rightarrow S U(3)_{c} \times S U(N-3) \times U(1)$, exhibiting the colour $S U(3)_{c}$. Therefore, $\left\langle\Phi_{3}\right\rangle=$ Diag. $\left(1,1,1,-\frac{3}{N-3}, \cdots,-\frac{3}{N-3}\right)$. Hence, this Higgs in the context of $F$ scheme gives $k=-\frac{3}{N-3}$, i.e. $k=-1,-3 / 4,-3 / 5,-1 / 2, \cdots$, while in $T^{\prime}$-scheme $k=\frac{6}{6-N}$, i.e. $k=\infty,-6,-3,-2, \cdots$ ( $k=\infty$ should be understood as $1 / k=0$, i.e. $A^{d}=0$ while $A^{e}$ is non-zero).

IV. There can be also adjoints with special VEV directions, having vanishing eigenvalues towards the $S U(2)_{w}$ components, say $\left\langle\Phi_{0}\right\rangle=\operatorname{Diag} \cdot\left(1,1,1,0,0,-\frac{3}{N-5}, \cdots,-\frac{3}{N-5}\right)$. It is natural to use such adjoints for the doublet-triplet splitting [26]. Obviously, this would give $k=0$ 
in either $F$ - or $T^{\prime}$-like schemes. Analogously, there can be adjoints with vanishing $S U(3)_{c}$ components, which would lead to $k=\infty$ in $F$-like scheme and $k=2$ in $T^{\prime}$-like scheme.

Therefore, in the next, while searching the realistic ansätze, we shall scan all possible Clebsch values for $k_{n}$ or $1 / k_{n}$ among $0, \pm 1, \pm 2, \pm 3$ plus the above $N$-dependent expressions (for all possible $N)$.四

It should be remarked that within this wide variety, we find only three acceptable solutions which characterise three different ansätze. We anticipate them here:

$$
\begin{array}{lll}
\operatorname{ansatz} & \mathbf{A} & k_{1}=k_{2}=2, \quad k_{3}=1, \\
\operatorname{ansatz} \mathbf{B} & k_{1}=k_{3}=-3, \quad k_{2}=0 \\
\text { ansatz } \mathbf{C} & k_{1}=-3, \quad k_{2}=0, \quad k_{3}=2
\end{array}
$$

We shall come back to this point in Sect. 4.3.

\section{The $S O(10) \times S U(3)_{H}$ model}

In the context of $S O(10)$ model all fermion representations (22) are unified into 16-plets $\psi_{i}$, where $i=1,2,3$ is a family $\left(S U(3)_{H}\right)$ index. In terms of the $S U(5)$ subgroup, the fermion content is represented as $\psi_{i}=(\overline{5}+10+1)_{i}$. So, in addition to the superfields $\overline{5}_{i}$ and $10_{i}(22)$, $\psi_{i}$ contain also the $S U(5)$ singlets $1_{i}$, usually referred to as $\mathrm{RH}$ neutrinos.

The $S O(10)$ symmetry can be spontaneously broken to $S U(5)$ by the Higgs supermultiplets $C \sim 16, \bar{C} \sim \overline{16}$, having the VEVs towards the singlet components. The $S U(5)$ symmetry can be further reduced down to $S U(3) \times S U(2) \times U(1)$ by Higgses in tensor representations 54 and 45. As for the MSSM Higgs doublets $H_{u, d}$ needed for breaking the electroweak symmetry and generating the fermion masses, they are contained in a single superfield $\phi \sim 10 .{ }^{-6}$ It is well known that the $S O(10)$ framework also offers the possibility to work out the basic problem of the doublet-triplet splitting which has to be unavoidably adressed in any realistic grand unified theory. In particular, one can implement the so called missing VEV mechanism [27] which can be justified by additional symmetry reasons (see [28, 29] and references therein). It allows to achieve the mass splitting between the components of the Higgs 10-plet so that the doublets $H_{u, d}$ remain massless while their colour triplet partners get masses of the order of the GUT scale, without unnatural fine tuning of the Higgs superpotential parameters.

Since the 10-plet $\phi$ can couple only to the symmetric combination of the fermion 16plets, the higher-order operators inducing the fermion masses can only involve the (anti)sextet horizontal Higgs $S$ (cfr. the $S U(5)$ case (24) where also triplet Higgses $A$ can contribute). Hence, in the context of the $S O(10) \times S U(3)_{H}$ model the operators (24) are represented as:

$$
\mathcal{O} \sim \frac{S^{i j}}{M} \psi_{i} \psi_{j} \phi
$$

where, as in Sect. 2, the flavour scale $M$ can be of the order of the GUT scale $M_{G} \sim 10^{16}$ $\mathrm{GeV}$, and $Y_{t} \sim 1$ implies that $\mathcal{S}_{3} \sim M$. One can still define $\mathbf{S}=M^{-1}\langle S\rangle$. Thus, in the

\footnotetext{
${ }^{15}$ In the context of $S U(N)$ models containing $S U(5) \times S U(3)_{H},(N \geq 8)$, the large value of the horizontal Clebsches $b, b^{\prime}$ could be similarly obtained if also the $I+\Sigma \sim(1+8)$-like combinations emerge from the VEV of the "big" adjoint breaking $S U(N)$ down to $S U(N-1) \times U(1)$, i.e. $b=N-1$.

16 We remind the content of $S O(10)$ multiplets with respect to the $S U(5)$ subgroup: $16=\overline{5}+10+1$, $\overline{16}=5+\overline{10}+1,54=24+15+\overline{15}, 45=1+24+10+\overline{10}$ and $10=\overline{5}+5$.
} 
low-energy limit this operator provides equal Yukawa matrices for all fermions, including that for the neutrino Dirac mass terms: $\mathbf{Y}_{u}, \mathbf{Y}_{d, e}, \mathbf{Y}_{\nu}^{D}=\mathbf{S}$. In addition, the operator

$$
\mathcal{O}_{R} \sim \frac{S^{i j}}{M^{2}} \psi_{i} \psi_{j} \bar{C}^{2}
$$

involving the Higgs 16-plet with a VEV $V_{C} \sim M$ induces the Majorana mass terms for the $\mathrm{RH}$ neutrinos: $\mathbf{M}_{R} \propto \mathbf{S}$. Thus, the effective operator for the neutrino masses emerges from the familiar see-saw mechanism, and it has a form (5) with $\mathbf{Y}_{\nu}=\eta \mathbf{Y}_{u}$, where $\eta \sim\left(M / V_{C}\right)^{2}$.

Therefore, in such a straightforward case we obtain a highly unrealistic pattern for the fermion masses and vanishing mixing angles in both the quark and lepton sectors. Recalling the discussion in Sect. 2.1, the way out from this situation is to invoke $S O(10)$ and $S U(5)$ breaking Higgses (45- and 54-plets) in the effective operators for fermion masses, as well as the $S U(3)_{H}$ octet Higgs for breaking the horizontal symmetry.

In this section we present a consistent $S O(10) \times S U(3)_{H}$ model to motivate the desired Yukawa textures (20). By explicitly specifying the extra symmetries, generically denoted by $\mathcal{G}$ in the previous section, we will show, for example, how the Clebsch structure of the ansatz $\mathbf{B}$ may be obtained. Interestingly, the same symmetry reasons can be utilized for solving the problem of the doublet-triplet splitting via the missing VEV mechanism.

Let us describe the ingredients of the model. For the sake of simplicity, we assume that all 'vertical' Higgses are singlets of $S U(3)_{H}$ and vice versa, all 'horizontal' Higgses are singlets of $S O(10)$. In particular, the 'vertical' sector of $S O(10)$ Higgses includes chiral superfields in the representations 54, 45, 16 and $\overline{16}$, needed for the $S O(10)$ symmetry breaking down to $S U(3) \times S U(2) \times U(1)$, and 10-plets for the electroweak symmetry breaking and the fermion mass generation. The "horizontal" sector of $S U(3)_{H}$ Higgses includes the triplet, sextet and octet representations. Namely, as in the previous section, we introduce one anti-sextet $S \sim \overline{6}$, three triplets $A_{1,2,3} \sim 3$ (plus the conjugated "spectator" superfields $\bar{S} \sim 6$ and $\bar{A}_{1,2,3} \sim \overline{3}$ ), and the $S U(3)_{H}$ adjoint $\Sigma \sim 8$. In addition, there can be some fields which are singlets under both $S O(10)$ and $S U(3)_{H}$.

Besides the matter superfields $\psi_{i} \sim(16,3)$ we introduce a number of vector-like representations containing the fragments like (28), (29) and (37) which are needed for the fermion mass generation. Therefore, these should be matter superfields in $S O(10)$ representations $16+\overline{16}$, 10 or singlets, to be specified below. These states mediate the see-saw like diagrams and hence the quark and lepton Yukawa structures emerge after integrating them out.

We invoke the additional symmetry in the form $\mathcal{G}=U(1)_{A} \times Z_{6} \times \mathcal{R}$, where $U(1)_{A}$ is an anomalous gauge symmetry [30] implemented in the same spirit as in ref. [29], $\mathcal{R}$ is a discrete symmetry (as in Sect. 2.2) under which all superfields in the theory as well as the superpotential change the sign, and $Z_{6}$ is a discrete symmetry acting on the superfields as $\varphi_{n} \rightarrow \varphi_{n} \exp \left(\frac{\pi}{3} i Q_{n}\right)$.

The superfield content of the model with respect to $S O(10) \times S U(3)_{H}$ is given below, and the superfield charge assignment $[\mathcal{Q}, Q]$ with respect to $U(1)_{A}$ and $Z_{6}$ symmetries, respectively, is shown in subscript. Namely, we assume that the Higgs sector contains the following superfields:

(i) the $S O(10)$ representations

$$
\begin{array}{llll}
\Theta \sim(54,1)_{[0,0]}, & \Phi \sim(45,1)_{[0,3]}, & \Omega \sim(45,1)_{[0,2]}, & \Omega^{\prime} \sim(45,1)_{[0,1]}, \\
C \sim(16,1)_{[-1 / 2,1]}, & \bar{C} \sim(\overline{16}, 1)_{[1 / 2,3]}, & \phi \sim(10,1)_{[-1,0]}, & \phi^{\prime} \sim(10,1)_{[1,3]},
\end{array}
$$


(ii) the $S U(3)_{H}$ representations

$$
\begin{array}{llll}
S \sim(1, \overline{6})_{[0,0]}, & A_{1} \sim(1,3)_{[0,1]}, & A_{2} \sim(1,3)_{[0,-2]}, & A_{3} \sim(1,3)_{[0,1]}, \\
\bar{S} \sim(1,6)_{[0,0]}, & \bar{A}_{1} \sim(1, \overline{3})_{[0,3]}, & \bar{A}_{2} \sim(1, \overline{3})_{[0,0]}, & \bar{A}_{3} \sim(1, \overline{3})_{[0,3]},
\end{array}
$$

and $\Sigma \sim(1,8)_{[-2,0]}$,

(iii) and the gauge singlets

$$
Y \sim(1,1)_{[0,0]}, \quad Z \sim(1,1)_{[0,2]}, \quad \bar{Z} \sim(1,1)_{[0,-2]} \quad I \sim(1,1)_{[-2,0]} .
$$

It is convenient to split the complete Higgs superpotential $W_{\text {Higgs }}$ consistent with the above symmetries, into the following parts:

$$
\begin{aligned}
W_{1}= & \Lambda^{2} Y+Y^{3}+Y \Theta^{2}+\Theta^{3}+(Y+\Theta) \Phi^{2}+Y Z \bar{Z}+Y S \bar{S} \\
& +Z^{3}+\bar{Z}^{3}+Z \Omega^{2}+Z C \bar{C}+\Omega C \bar{C}+\bar{Z} \Omega^{\prime 2}+\Phi \Omega \Omega^{\prime} \\
& +S^{3}+\bar{S}^{3}+Z A_{n} \bar{A}_{n}+A_{1} A_{2} A_{3}+\bar{A}_{1} \bar{A}_{2} \bar{A}_{3}, \\
W_{2}= & \Phi \phi \phi^{\prime}+I \phi^{\prime 2}+\phi \bar{C}^{2},
\end{aligned}
$$

(all $S O(10)$ and $S U(3)_{H}$ index contractions are left understood). Here order 1 coupling constants are implied in the trilinear terms, and $\Lambda$ is a mass parameter of the order of the GUT scale, $M_{G} \sim 10^{16} \mathrm{GeV}$.

An important role is played by the D-term of the anomalous $U(1)_{A}$ symmetry

$$
D_{A}=\mathcal{M}^{2}+\sum \mathcal{Q}_{n}\left|\varphi_{n}\right|^{2}, \quad \mathcal{M}^{2}=\frac{\operatorname{Tr} \mathcal{Q}}{192 \pi^{2}} M_{\mathrm{str}}^{2}
$$

where the sume runs over all scalar components $\varphi_{k}$ in the theory and $\mathcal{Q}$ are their $U(1)_{A}$ charges. Therefore, the spontaneous breaking scale of the $U(1)_{A}$ symmetry is naturally related to the string scale $M_{\text {str }} \sim 10^{17-18} \mathrm{GeV}$ with a coefficient determined by the charge content of the superfields in the game.

Notice that $\Lambda$ is the unique mass parameter contained in the superpotential (67), while another mass scale in the theory comes from the Fayet-Iliopulos term $\mathcal{M}^{2}$. Therefore, the VEV magnitudes of all fields into the game should be determined by these two mass scales, which in addition can be both of the order of the GUT scale. In particular, the Higgs fields participating in the superpotential $W_{1}$ can get $\sim \Lambda$ VEVs violating the $S O(10)$ and $S U(3)_{H}$ symmetries. In fact, the linear term $\Lambda^{2} Y$ induces the VEVs of the singlets $Y, Z, \bar{Z}$ which in turn play the role of mass terms for the superfields in non-trivial representations like $\Omega, \Omega^{\prime}, \Phi, \Theta$, etc. Thus, with an accuracy of $\mathcal{O}(1)$ coupling constants in the superpotential terms (67), the magnitude of all non-zero VEVs is $\sim M_{G}$. As for the fields $I$ and $\Sigma$, they can get VEV of the order $\mathcal{M}$ from the anomalous D-term (68). We assume that the trace $\operatorname{Tr} \mathcal{Q}$ of the $U(1)_{A}$ charges over all superfields is positive: namely, the superfields presented in (64) and (66) plus the octet $\Sigma$ contribute as $\operatorname{Tr} \mathcal{Q} \sim 10^{2}$. (The theory may include also other superfields with charges arranged so that the Green-Schwarz cancellation mechanism is at work, but this does not affect the orders of magnitude in our consideration). Therefore, $\mathcal{M}$ can naturally be about the GUT scale $M_{G}$ or a bit larger. One has also to remark that the anomalous term fixes

\footnotetext{
${ }^{17}$ As far as R-parity is concerned, generally it is not automatic in $S O(10)$ models involving the Higgs 16 -plets. However, in the context of our model one can easily verify that all dangerous R-violating terms are suppressed by the $\mathcal{G}$ symmetry.
} 
only the overall VEV combination $\langle I\rangle^{2}+\langle\Sigma\rangle^{2}$, but not each term separately. The VEV values of $I$ and $\Sigma$ will be fixed by the soft supersymmetry breaking terms, as well as orientation of $\langle\Sigma\rangle$ with respect to the VEVs of other horizontal scalars, $S$ and $A_{1,2,3}$, in the spirit discussed in sect. 2.2. This means that the horizontal fields should contain some light fragments, with masses order $\mathrm{TeV}$, which, on the other hand, do not lead to phenomenological problems (see e.g. discussion in ref. [23]). The only important point is that, due to the simple structure of the $S U(3)_{H}$ group, the VEV of $\Sigma$ in either case would acquire the simplest possible configuration $\langle\Sigma\rangle \propto \operatorname{Diag}(1,1,-2)$.

Remarkably, the terms contained in the superpotential $W_{1}$ provide the pattern needed to break $S O(10)$ to the standard model group. In particular, there exists a solution for the 16-plet $C, \bar{C}$ VEVs which breaks $S O(10)$ down to $S U(5):\langle C\rangle=\langle\bar{C}\rangle=V_{C} \cdot|+,+,+,+,+\rangle$ (in terms of the corresponding Cartan sub-algebra generators). The condition $\langle C\rangle=\langle\bar{C}\rangle$ follows from the vanishing of the SO(10) D-terms. The 54 and 45 Higgses are needed to subsequently break $S U(5)$ down to $S U(3) \times S U(2) \times U(1)$. The necessary VEVs are given as:

$$
\begin{aligned}
& \langle\Theta\rangle=V_{\Theta} \cdot \operatorname{Diag}(1,1,1,-3 / 2,-3 / 2) \otimes \sigma_{0}, \\
& \langle\Phi\rangle=V_{\Phi} \cdot \operatorname{Diag}(1,1,1,0,0) \otimes \sigma, \\
& \langle\Omega\rangle=V_{\Omega} \cdot \operatorname{Diag}(1,1,1,1,1) \otimes \sigma, \\
& \left\langle\Omega^{\prime}\right\rangle=0,
\end{aligned}
$$

where

$$
\sigma_{0}=\left(\begin{array}{cc}
1 & 0 \\
0 & 1
\end{array}\right), \quad \sigma=\left(\begin{array}{cc}
0 & 1 \\
-1 & 0
\end{array}\right) .
$$

One can easily verify that these VEVs are consistent with a supersymmetric vacuum and their configurations are not affected by some unknown factors. In particular, in the 54-plet $\Theta$ only its 24 fragment (in terms of $S U(5)$ subgroup) acquires VEV and, noticeably, into the only phenomenologically allowed direction which induces the $S O(10)$ symmetry breaking down to the Pati-Salam subgroup: $S O(10) \rightarrow S U(4) \times S U(2) \times S U(2)^{\prime}$. The coupling of the 45-plet $\Phi$ to $\Theta$ in the superpotential forces the VEV of the former to lie in the $B-L$ direction, as a result of a combination of its $S U(5) 1$ and 24 fragments, with exact zeros on the last two components, as it is demanded by the missing VEV mechanism [27, 28]. 18 As for the other 45 -plet $\Omega$, it is coupled to the 16 -plets $C, \bar{C}$, but does not couple to the 54-plet $\Theta$. Because of this, its VEV is directed entirely towards the $S U(5)$-singlet direction, with a vanishing VEV towards the 24 fragment. To And finally, the third 45-plet $\Omega^{\prime}$ does not couple neither to $\Theta$ nor to $C, \bar{C}$, and thus it should have a vanishing VEV.

It is also important that all non-singlet (under the standard model group) fragments in the $S O(10)$-breaking superfields get masses of the order of $M_{G}$. In such a case, no light fragment (with mass $\ll M_{G}$ ) is left behind which could affect the gauge coupling unification at the scale $M_{G}$. The presence of the term $\Phi \Omega \Omega^{\prime}$ is crucial for achieving this result [28], since otherwise, in its absence, the theory would have flat directions towards non-singlet fragments which are

\footnotetext{
${ }^{18}$ As we will see below in the analysis of the Yukawa sector, such a VEV structure of $\Phi$ is also important for obtaining the Clebsch factors needed for the ansatz $\mathrm{B}$, namely $k_{1,3}=-3$ and $k_{2}=0$.

${ }^{19}$ This is also relevant for the fermion Clebsch structures, because a $S U(5)$-singlet VEV - namely, the combination $\langle Z+\Omega\rangle$ - participating in the fermion mass generation (see below, diagrams in Fig. 4) does not induce uncontrollable corrections to the Clebsch factors.
} 
related to extra global symmetry in the superpotential. Remarkably, this term does not affect the VEV pattern of 45-plets while lifting the dangerous flat directions.

Finally, the term $W_{1}$ in (67) is also responsible for the horizontal $S U(3)_{H}$ breaking via the VEVs of $S$ and $A_{1,2,3}$ fields (see (26) and (27)) and its features are elaborated in Appendix A. Notice, that even if all coupling constants are taken real in the superpotential, non-trivial $\mathrm{CP}$-violating phase in these VEVs could appear in case of solutions with different phases for the singlet VEVs $\langle Y\rangle$ and $\langle Z\rangle$, in the spirit discussed in Appendix B.

Let us address now the doublet-triplet splitting problem. As well-known, the VEV of the 54-plet $\Theta$ guarantees the VEV of the 45-plet $\Phi$ to be towards the $B-L$ direction, $\langle\Phi\rangle \propto$ $\operatorname{Diag}(1,1,1,0,0) \times \sigma$. On the other hand, the superpotential $W_{2}$ contains the necessary terms to incorporate the missing VEV mechanism for the doublet-triplet splitting. In addition, they also induce the mixing between the $\overline{5}$ fragments in $\phi$ and $C$ through the large $S U(5)$ conserving VEV $V_{C}=\langle C\rangle$. All this can be more easily represented in terms of the following mass matrix:

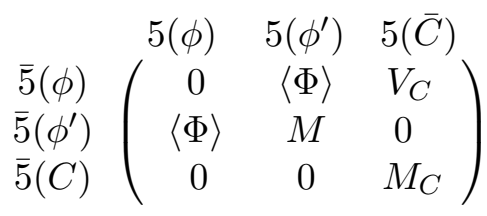

where $M \sim\langle I\rangle$ and $M_{C} \sim\langle Z+\Omega\rangle$ is the mass of the 5-plet fragments contained in $C, \bar{C}$. Owing to the form of the $\operatorname{VEV}\langle\Phi\rangle$ in (69), non-zero entries are induced only between the triplet fragments in $\phi$ and $\phi^{\prime}$ while the corresponding entries for the doublet fragments vanish. Therefore, all the triplets get masses of the order of $M_{G}$, while two combinations of the doublet components, to be identified with the MSSM Higgs doublets $H_{u, d}$, remain light. The doublet $H_{u}$ comes entirely from $H \equiv 5(\phi)$, and so no portion of it is contained in $5(\bar{C})$ or $5\left(\phi^{\prime}\right)$. As regards $H_{d}$, it emerges from the combination of $\overline{5}$ fragments in $\phi$ and $C$. Namely, $\bar{H}=c_{\omega} \overline{5}(C)-s_{\omega} \overline{5}(\phi)$, with $\tan \omega \sim M_{C} / V_{C}$ (see also [31]).

Let us discuss now the fermion mass generation mechanism. Apart from the already familiar $\psi \sim(16,3)_{[-1 / 2,0]}$, let us introduce the following vector-like fermions:

(I) 16-plets

$$
\Psi \sim(16, \overline{3})_{[3 / 2,0]}, \quad \bar{\Psi} \sim(\overline{16}, 3)_{[1 / 2,0]} ; \quad \Psi^{\prime} \sim(16,3)_{[3 / 2,3]}, \quad \bar{\Psi}^{\prime} \sim(\overline{16}, \overline{3})_{[1 / 2,3]},
$$

(II) 10-plets

$$
\begin{array}{rlrl}
t \sim(10,3)_{[-1,3]}, & \bar{t} \sim(10, \overline{3})_{[1,-1]}, & t_{1} \sim(10,3)_{[1,2]}, & \bar{t}_{1} \sim(10, \overline{3})_{[-1,2]}, \\
& t_{2} \sim(10,3)_{[-1,0]}, & \bar{t}_{2} \sim(10, \overline{3})_{[1,0]}
\end{array}
$$

(III) and singlets

$$
N \sim(1, \overline{3})_{[0,3]}, \quad \bar{N} \sim(1,3)_{[0,3]} .
$$

Let us start by discussing the role played by the additional fermion 10-plets $t_{i}=(\overline{5}+5)_{i}$, $\bar{t}^{i}=(\overline{5}+5)^{i}$. All the symmetries of the model allow in particular the following terms:

$$
C \psi \bar{t}+\bar{Z} t \bar{t}
$$

Correspondingly, the mass matrix connecting the matter states reads as:

$$
\begin{gathered}
\overline{5}\left(\psi_{i}\right) \\
\overline{5}\left(t_{i}\right) \\
\overline{5}\left(\bar{t}^{i}\right)
\end{gathered}\left(\begin{array}{cc}
0 & 5\left(\bar{t}^{i}\right) \\
0 & \langle\bar{Z} \\
\langle\bar{Z}\rangle & 0
\end{array}\right),
$$


(a)

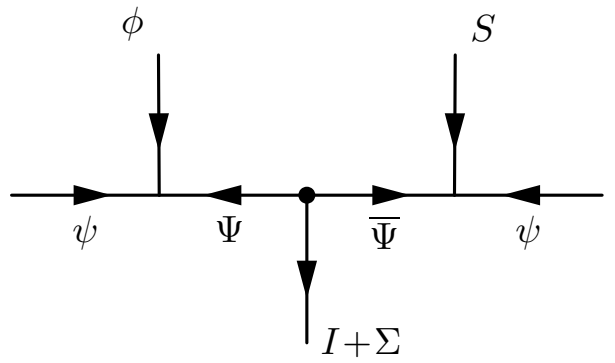

(b)

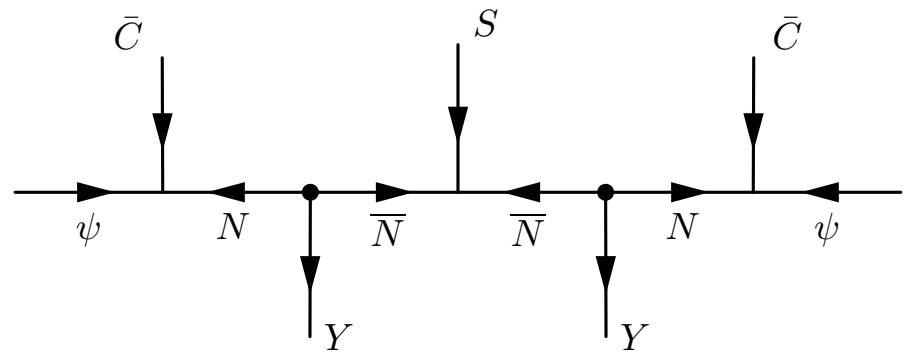

Figure 3: The see-saw diagrams giving rise to the effective higher order operators for the symmetric contributions to the charged fermion Yukawa matrices and to the neutrino Dirac mass term $\mathbf{Y}_{\nu}^{D}-$ diagram (a), and to the Majorana mass matrix $\mathbf{M}_{R}$ of the $\mathrm{RH}$ neutrinos 1 contained in $\psi$ - diagram (b). In $S U(5)$ language the relevant fragments are $\psi=\overline{5}+10+1, \phi=5+\overline{5}$, and the singlet component of $\bar{C}$ with VEV $V_{C}$.

which shows that the light states $\overline{5}_{i}$ are composed by a combination of $\overline{5}$ fragments in $\psi_{i}$ and $t_{i}$ i.e. $\overline{5}_{i}=-s_{\theta} \overline{5}(\psi)_{i}+c_{\theta} \overline{5}(t)_{i}$, where the angle $\theta$ is defined as $\tan \theta=\langle\bar{Z}\rangle / V_{C}$, while the orthogonal combinations $\overline{5}_{i}^{\prime}=c_{\theta} \overline{5}(\psi)_{i}+s_{\theta} \overline{5}(t)_{i}$ and $\overline{5}(\bar{t})^{i}$ are heavy states.

Now we are in position to fix the fermion mass pattern which emerges in our theory. The diagonal entries in the Yukawa matrices are generated by the following superpotential terms:

$$
\begin{aligned}
& W_{\Psi}=\psi \phi \Psi+\Psi(I+\Sigma) \bar{\Psi}+\bar{\Psi} S \psi \\
& W_{N}=\psi \bar{C} N+N Y \bar{N}+S \bar{N}^{2},
\end{aligned}
$$

which through the see-saw like diagrams shown in Fig. 3, give rise to the effective operators like (62) and (63). For an easy identification of the contributions to the Yukawa matrices, the terms in $W_{\Psi}$ and $W_{N}$ can be rewritten according to their $S U(5)$ decomposition:

$$
\begin{aligned}
W_{\Psi}= & 10(\psi) 5(\phi) 10(\Psi)+\overline{5}(\psi) \overline{5}(\phi) 10(\Psi)+\overline{10}(\bar{\Psi}) S 10(\psi)+10(\Psi)(I+\Sigma) \overline{10}(\bar{\Psi})+ \\
& 10(\psi) \overline{5}(\phi) \overline{5}(\Psi)+5(\bar{\Psi}) S \overline{5}(\psi)+\overline{5}(\Psi)(I+\Sigma) 5(\bar{\Psi})+ \\
& \overline{5}(\psi) 5(\phi) 1(\Psi)+1(\bar{\Psi}) S 1(\psi)+1(\Psi)(I+\Sigma) 1(\bar{\Psi}), \\
W_{N}= & 1(\psi) 1(\bar{C}) N+N Y \bar{N}+S \bar{N}^{2} .
\end{aligned}
$$

Therefore, as already discussed in Sect. 2.1, the VEV combination $\langle I+\Sigma\rangle$ provides the $S U(3)_{H}$ breaking and leads to the $S U(2)_{H}$ invariant form of the heavy fermion mass matrix $\mathbf{M}_{\Psi}=M \mathbf{b}^{\prime}$, where $\mathbf{b}^{\prime}=\operatorname{Diag}\left(1,1, b^{\prime}\right)$ and $M \sim \mathcal{M}$. The relevant Yukawa terms for the light fermions emerge after integrating out the heavy states. In particular, for the Yukawa coupling matrix of up quarks (which are entirely contained in $10(\psi)$ fragment) to the Higgs doublet $H_{u} \subset 5(\phi)$ we obtain $\mathbf{Y}_{u}=\mathbf{M}_{\Psi}^{-1}\langle S\rangle=\mathbf{b}^{-1} \mathbf{S}$, where $\mathbf{S} \equiv M^{-1}\langle S\rangle$ and it is diagonal once the VEV $\langle S\rangle$ is 
(a)

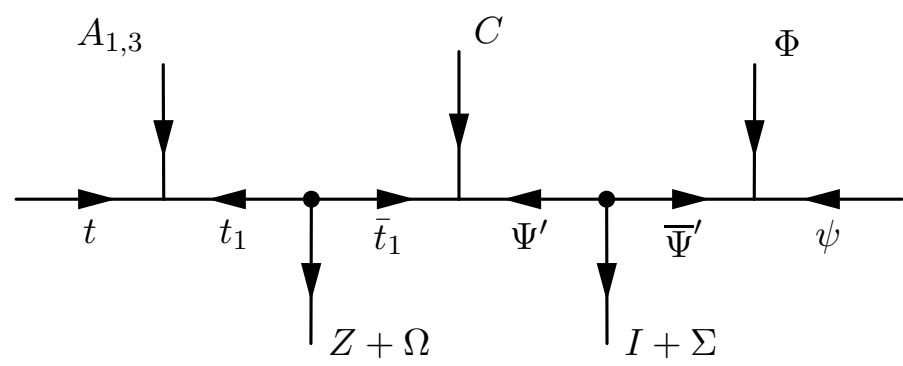

(b)

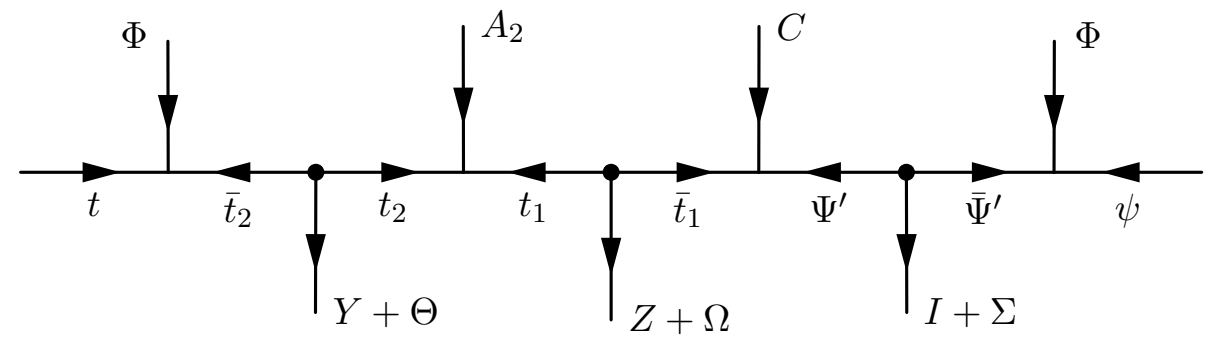

Figure 4: Diagrams giving rise to the effective higher-order operators for the non-diagonal contributions to $\mathbf{Y}_{e, d}$. The relevant contributions of these diagrams involve $H_{d} \subset \overline{5}(C)$ and $\overline{5}_{i} \subset \overline{5}\left(t_{i}\right)$ states.

chosen as (26). On the other hand, recalling that $\overline{5}(\psi)$ contains the light $\overline{5}$-plet with a weight $s_{\theta}$ and the Higgs $\overline{5}(\phi)$ contains $H_{d}$ with a weight $s_{\omega}$, the terms (78) provide also the diagonal contributions to the down quark and charged lepton Yukawa matrices $\mathbf{Y}_{d, e}^{\text {diag }}=s_{\theta} s_{\omega} \mathbf{b}^{\prime-1} \mathbf{S}$. From the decomposition (78) it is apparent that the (heavy fermion) exchange contributions to $\mathbf{Y}_{u}$ are of the $T$-scheme type (cfr. (35)) and those to $\mathbf{Y}_{d, e}^{\text {diag }}$ are of both $T$ - and $F$-scheme type (cfr. also (38)).

Finally, for the Dirac Yukawa matrix of neutrinos we obtain $\mathbf{Y}_{\nu}^{D}=s_{\theta} \mathbf{b}^{\prime-1} \mathbf{S}$. On the other hand, by considering the terms in $W_{N}$, we see that the $\operatorname{VEV}\langle Y\rangle$ induces the $S U(3)_{H}$ invariant masses between the states $N$ and $\bar{N}, \mathbf{M}_{N}=M_{N} \cdot \mathbf{1},(\mathbf{1}=\operatorname{Diag}(1,1,1))$ and $M_{N} \sim M_{G}$, and so the RH neutrinos $1(\psi)$ acquire the Majorana masses $\mathbf{M}_{R}=\mathbf{M}_{N}^{-2} V_{C}^{2}\langle S\rangle=\kappa M \mathbf{S}$, where $\kappa \sim\left(V_{C} / M_{N}\right)^{2} \sim 1$. Thus, as long as both Dirac and Majorana terms are present for neutrinos, the operator (5) emerges via the standard see-saw mechanism:

$$
\frac{\mathbf{Y}_{\nu}}{M_{L}}=\mathbf{Y}_{\nu}^{D} \mathbf{M}_{R}^{-1} \mathbf{Y}_{\nu}^{D}=\frac{s_{\theta}^{2}}{\kappa M} \mathbf{b}^{\prime-2} \mathbf{S}
$$

Therefore, both $\mathbf{Y}_{u}$ and $\mathbf{Y}_{\nu}$ are diagonal and we have:

$$
\mathbf{Y}_{\nu}=\eta \mathbf{b}^{\prime-1} \mathbf{Y}_{u}
$$

where $\eta=s_{\theta}^{2} / \kappa$.

Let us discuss now the origin of the off-diagonal entries in $\mathbf{Y}_{d, e}$, recalling that they have already got the diagonal contributions $\propto \mathbf{Y}_{u}$. We also remember that the off-diagonal entries should contain non-trivial Clebsches between quarks and leptons.

To this purpose, we recall that the light fermion $\overline{5}$-plet is also contained in $\overline{5}(t)$ with a weight $c_{\theta}$, while the doublet $H_{d}$ is contained in the fragment $\overline{5}(C)$ with a weight $c_{\omega}$. Then, the symmetry of the theory prescribes other superpotential couplings for the superfields $t$ and $C$, involving other (than $\Psi, \bar{\Psi}$ ) heavy fermions, so that the exchange of the latter induce the 
off-diagonal contributions to $\mathbf{Y}_{d, e}$ via the VEVs of the triplet horizontal Higgses $A_{1,2,3}$. The relevant diagrams 20 are shown in Fig. 4 . The diagram (a) provides the entries $(2,3)$ and $(1,2)$ as only the $S U(3)_{H}$ triplets $A_{1}$ and $A_{3}$ are allowed to have a direct coupling to the matter 10-plets $t_{i}$. In this case, the coupling of the 45 -plet $\Phi$ (with its VEV into the $B-L$ direction) to the 16-plets $\left(\psi\right.$ and $\left.\bar{\Psi}^{\prime}\right)$ gives rise to the Clebsch coefficients $k_{1,3}=-3$. We should also observe that these off-diagonal contributions are effectively induced by the exchange of the $S U(5) 10+$ $\overline{10}$ fragments contained in the vector-like states $\Psi^{\prime}+\bar{\Psi}^{\prime}$, according to the $T^{\prime}$-scheme (cfr. (40)).

The triplet $A_{2}$ is instead involved in the diagram (b) which therefore contributes to the $(1,3)$ entries. Because of the requirement of $\mathcal{G}$ invariance, this triplet cannot couple to the states $t_{i}$ but it couples to the heavy states $t_{2}$ which are mixed to the former through the VEV of the 45-plet $\Phi$. This occurs through the couplings on the left of the $A_{2}$ incoming-line in the diagram (b), while the part on the right side contains exactly the same couplings as that on the right of the $A_{1,3}$ line in diagram (a). However, the coupling of the 45-plet $\Phi$ with the fermion 10-plets, $t \Phi \bar{t}_{2}$, gives vanishing contribution to the lepton doublets contained in $\overline{5}(t)$ (similarly to what happens in the missing VEV mechanism for the doublet-triplet splitting) and therefore we obtain $k_{2}=0$. In either case of diagram (a) or (b), the $S U(3)_{H}$ breaking Clebsch $b$ emerges from the same combination $I+\Sigma$ (i.e. from the matrix $\mathbf{M}_{\psi}^{\prime} \sim M \mathbf{b}_{\Psi^{\prime}}=M \cdot \operatorname{diag}\left(1,1, b_{\Psi^{\prime}}\right)$ ) so that the 13 and 23 entries have the same asymmetry.

Thus, the final form of the down quark and charged lepton Yukawa matrices reads as:

$$
\mathbf{Y}_{d}=\rho \mathbf{Y}_{u}^{D}+\mathbf{b}^{-1} \mathbf{A}_{d}, \quad \mathbf{Y}_{e}^{T}=\rho \mathbf{Y}_{u}^{D}+\mathbf{b}^{-1} \mathbf{A}_{e},
$$

where the anti-symmetric matrices $\mathbf{A}_{d, e}$ have the form (50) with $k_{1,3}=-3$ and $k_{2}=0$, $\mathbf{b}=\operatorname{diag}(1,1, b)$ where $b=b_{\Psi^{\prime}}^{-1}$. In this way we have reproduced the Clebsch factors demanded by the ansatz $\mathbf{B}(60)$. The coefficient $\rho$ is identified with $s_{\omega} s_{\theta}$. It may be smaller than 1 and thus moderate or even small values of $\tan \beta$ can naturally be accommodated.

In conclusion, we have presented a complete $S O(10) \times S U(3)_{H}$ model in which the symmetry breaking VEV pattern as well as the form of the superpotential couplings needed to obtain the desired predictive mass texture for the fermions (specifically, the ansatz $\mathbf{B}$ presented in Sect. 2) are fully motivated by the additional $\mathcal{G}=U(1)_{A} \times Z_{6} \times \mathcal{R}$ symmetry. Interestingly, the missing VEV mechanism for the doublet-triplet splitting can be motivated on the basis of the same symmetry $\mathcal{G}$. The other Clebsch structures demanded by the ansätze $\mathbf{A}$ or $\mathbf{C}$ can be reproduced along similar lines by rearranging the charge assignments of the superfields in the game with respect to the $Z_{6}$ symmetry, however we will not show the corresponding models here.

\section{Phenomenological analysis}

This part is devoted to the phenomenological study of the ansätze suggested. First, we provide the corresponding expressions of the physical quantities - quark masses as well as mixing angles. Second, the criteria of the fit are presented. Finally the results are shown and discussed.

\footnotetext{
${ }^{20}$ For the sake of brevity we do not write down the relevant superpotential terms responsible for the generation of the off-diagonal Yukawa couplings: they can be read out directly from the diagrams in Fig. 4 .
} 


\subsection{Physical observables from the theoretical model}

We now deal with the explicit form for the Yukawa matrices $\mathbf{Y}_{e, d}$ in (51). In general the coupling constants and the VEVs present in the theory are complex. However, all the phases except three can be pulled out by field redefinitions and the Yukawa matrices can be presented in this form:"2

$$
\begin{gathered}
\mathbf{Y}_{u}=\left(\begin{array}{ccc}
Y_{u} & 0 & 0 \\
0 & Y_{c} & 0 \\
0 & 0 & Y_{t}
\end{array}\right), \quad \mathbf{Y}_{\nu}=\eta\left(\begin{array}{ccc}
Y_{u} & 0 & 0 \\
0 & Y_{c} & 0 \\
0 & 0 & \frac{Y_{t}}{b}
\end{array}\right), \\
\mathbf{Y}_{d}=\left(\begin{array}{ccc}
Y_{u t} D \mathrm{e}^{\mathrm{i} \zeta} & A \mathrm{e}^{\mathrm{i} \sigma} & C \\
-A \mathrm{e}^{\mathrm{i} \sigma} & Y_{c t} D \mathrm{e}^{\mathrm{i} \xi} & B \\
\frac{1}{b} C & \frac{1}{b} B & D
\end{array}\right), \quad \mathbf{Y}_{e}=\left(\begin{array}{ccc}
Y_{u t} D \mathrm{e}^{\mathrm{i} \zeta} & -k_{3} A \mathrm{e}^{\mathrm{i} \sigma} & \frac{1}{b} k_{2} C \\
k_{3} A \mathrm{e}^{\mathrm{i} \sigma} & Y_{c t} D \mathrm{e}^{\mathrm{i} \xi} & \frac{1}{b} k_{1} B \\
k_{2} C & k_{1} B & D
\end{array}\right),
\end{gathered}
$$

Here $D=\rho Y_{t}, Y_{u t}=Y_{u} / Y_{t}$ and $Y_{c t}=Y_{c} / Y_{t}$. Notice that in $\mathbf{Y}_{\nu} b^{\prime}=b$ has been taken for concreteness. As compared to the expressions in (51) the off-diagonal terms have been redefined as $A_{1}^{d}=B, A_{2}^{d}=C$ and $A_{3}^{d}=A$ and understood to be real.22

The following observations can be made. The 11 entry in the matrices $\mathbf{Y}_{e, d}$ is negligible and in the following we set it to zero. Indeed, the ratio $Y_{u} / Y_{t}$ is two orders of magnitude less than $Y_{e} / Y_{\tau}$ and $Y_{d} / Y_{b}$ (cfr. Table 1) and thus its contribution in $Y_{d, e}$ is irrelevant.

As regards the 22 entry, this also leads to small corrections - the ratio $Y_{c} / Y_{t}$ is one order of magnitude less than $Y_{s} / Y_{b}$ (cfr. Table 1). However, we find that these corrections are relevant on comparing with the present precision in the CKM angles. So this contribution in $Y_{d, e}$ cannot be neglected.

In general case the phases in (83) are arbitrary. However, they can have specific values in the context of the spontaneous $\mathrm{CP}$ violation. Namely, one can assume that the original theory has an exact CP invariance, i.e. all couplings in the Yukawa and Higgs sectors can be simultanoulsy made real by phase redefinitions of the superfields. However, CP invariance can be spontaneously broken by non-vanishing phases of some VEVs in the theory. In particular, as we show in Appendix B, the triplets $A_{n}$ can have complex phases.

For example, one can choose a basis where the VEVs of the sextet $S, \mathcal{S}_{1,2,3}$ are real. Thus, in this basis all three diagonal entries of $\mathbf{Y}_{d}$ in (51) are real. For definiteness, let us take them also to be positive. On the other hand, the off-diagonal entries keep on the phases originated from the VEVs of $A_{n}$. As we show in Appendix B, these VEVs can exhibit a spontaneous $\mathrm{CP}$ violation when the product $A_{1} A_{2} A_{3}$ is imaginary. In particular, if all entries $A_{1,2,3}$ are imaginary, the matrix $\mathbf{Y}_{d}$ in (51), once recasted in the form (83), implies the phase assignment $\xi=\pi, \sigma=\pi / 2$. In another possible case, when $A_{1,2}$ are real but $A_{3}$ is imaginary, one has instead $\xi=0$ and $\sigma=\pi / 2$. In principle, the spontaneous CP breaking could provide some other interesting phase assignments such as $\xi$ and $\sigma$ have some "inverse $\pi$-fold" values like $2 \pi / 3, \pi / 4$, etc. In the following, to be more general, we keep the phases $\xi$ and $\sigma$ arbitrary, however we shall pay special attention to those specific phase values which might be obtained in the context of the spontaneous $\mathrm{CP}$ violation.

\footnotetext{
${ }^{21}$ As far as $\mathbf{Y}_{u}$ and $\mathbf{Y}_{\nu}$ are diagonal, these phase transformations are not relevant for the form of the mixing matrices $V_{q, l}$ and we do not show them.

${ }^{22}$ In general the relative "lepton versus quark" Clebsches $k_{n}=A_{n}^{e} / A_{n}^{d}$ can be complex, however in the following they are taken real.
} 
Let us now proceed our analysis. The $(1,3)$ and $(3,1)$ entries in $\mathbf{Y}_{d, e}$ can be simultaneously rotated away by orthogonal and phase transformations in the 12-flavour space:

$$
\begin{array}{ll}
P_{-} \tilde{O}_{12}^{d T} \mathbf{Y}_{d} \tilde{O}_{12}^{d} P_{+}=\tilde{\mathbf{Y}}_{d}, & P_{+} \tilde{O}_{12}^{e T} \mathbf{Y}_{e} \tilde{O}_{12}^{e} P_{-}=\tilde{\mathbf{Y}}_{e}, \\
P_{ \pm}=\left(\begin{array}{ccc} 
\pm \mathrm{e}^{\mathrm{i} \sigma} & 0 & 0 \\
0 & 1 & 0 \\
0 & 0 & 1
\end{array}\right), & \tilde{O}_{12}^{d(e)}=\left(\begin{array}{ccc}
\tilde{c}_{12}^{d(e)} & \tilde{s}_{12}^{d(e)} & 0 \\
-\tilde{s}_{12}^{d(e)} & \tilde{c}_{12}^{d(e)} & 0 \\
0 & 0 & 1
\end{array}\right),
\end{array}
$$

where $\tilde{c}_{12}^{d(e)}=\cos \tilde{\theta}_{12}^{d(e)}$ etc. and it is taken $\tan \tilde{\theta}_{12}^{d}=C / B$ and $\tan \tilde{\theta}_{12}^{e}=\frac{k_{2}}{k_{1}} \tan \tilde{\theta}_{12}^{d}$. Notice that these rotations do not affect the $(1,2)$ and $(2,1)$ elements as the Yukawa matrices are antisymmetric in the 12 block. On the contrary the 23 block gets modified and by further re-defining everywhere the parameter $B$ as $\tilde{B} \equiv \frac{B}{\tilde{c}_{12}^{d}}$, the matrices become:

$$
\tilde{\mathbf{Y}}_{d}=\left(\begin{array}{ccc}
0 & -A & 0 \\
-A & Y_{c t} D \mathrm{e}^{\mathrm{i} \xi} & \tilde{B} \\
0 & \frac{1}{b} \tilde{B} & D
\end{array}\right), \quad \tilde{\mathbf{Y}}_{e}=\left(\begin{array}{ccc}
0 & -k_{3} A & 0 \\
-k_{3} A & Y_{c t} D \mathrm{e}^{\mathrm{i} \xi} & \frac{1}{b} \tilde{k}_{1} \tilde{B} \\
0 & \tilde{k}_{1} \tilde{B} & D
\end{array}\right)
$$

where $\tilde{k}_{1}=k_{1} \sqrt{\left(\tilde{c}_{12}^{d}\right)^{2}+\left(\tilde{s}_{12}^{d} k_{2} / k_{1}\right)^{2}}$ Finally, these matrices $\tilde{\mathbf{Y}}_{e}$ and $\tilde{\mathbf{Y}}_{d}$ can be diagonalized by bi-unitary transformations as in eq. (6) with the matrices $U_{e, d}$ and $U_{e, d}^{\prime}$ understood as:

$$
\begin{aligned}
& U=U_{32} U_{13} U_{12} \equiv \\
& \left(\begin{array}{ccc}
1 & 0 & 0 \\
0 & c_{23} & s_{23} \mathrm{e}^{i \phi_{23}} \\
0 & -s_{23} \mathrm{e}^{-i \phi_{23}} & c_{23}
\end{array}\right)\left(\begin{array}{ccc}
c_{13} & 0 & -s_{13} \mathrm{e}^{-i \phi_{13}} \\
0 & 1 & 0 \\
s_{13} \mathrm{e}^{i \phi_{13}} & 0 & c_{13}
\end{array}\right)\left(\begin{array}{ccc}
c_{12} & s_{12} \mathrm{e}^{i \phi_{12}} & 0 \\
-s_{12} \mathrm{e}^{-i \phi_{12}} & c_{12} & 0 \\
0 & 0 & 1
\end{array}\right) .
\end{aligned}
$$

Therefore, the CKM matrix is $V_{q}=\tilde{O}_{12}^{d} P_{+} U_{d}$ and is parameterised as

$$
V_{q}=\left(\begin{array}{ccc}
\tilde{c}_{12}^{d} e^{-i \sigma} v_{u d}+\tilde{s}_{12}^{d} v_{c d} & \tilde{c}_{12}^{d} e^{-i \sigma} v_{u s}+\tilde{s}_{12}^{d} v_{c s} & \tilde{c}_{12}^{d} e^{-i \sigma} v_{u b}+\tilde{s}_{12}^{d} v_{c b} \\
\tilde{c}_{12}^{d} v_{c d}-\tilde{s}_{12}^{d} e^{-i \sigma} v_{u d} & \tilde{c}_{12}^{d} v_{c s}-\tilde{s}_{12}^{d} e^{-i \sigma} v_{u s} & \tilde{c}_{12}^{d} v_{c b}-\tilde{s}_{12}^{d} e^{-i \sigma} v_{u b} \\
v_{t d} & v_{t s} & v_{t b}
\end{array}\right)
$$

where by $v_{u d}, v_{u s}$ etc. with lower $v$ we mean the matrix elements of the quark mixing $U_{d}$ as defined in eq. (7) and parameterised as in (8). Analogously, the effective leptonic mixing matrix is $V_{l}=U_{e}^{\dagger} P_{-}^{\dagger} \tilde{O}_{12}^{e T}$ :

$$
V_{l}=\left(\begin{array}{ccc}
-\tilde{c}_{12}^{e} e^{i \sigma} v_{e 1}+\tilde{s}_{12}^{e} v_{e 2} & \tilde{c}_{12}^{e} v_{e 2}+\tilde{s}_{12}^{e} e^{i \sigma} v_{e 1} & v_{e 3} \\
-\tilde{c}_{12}^{e} e^{i \sigma} v_{\mu 1}+\tilde{s}_{12}^{e} v_{\mu 2} & \tilde{c}_{12}^{e} v_{\mu 2}+\tilde{s}_{12}^{e} e^{i \sigma} v_{\mu 1} & v_{\mu 3} \\
-\tilde{c}_{12}^{e} e^{i \sigma} v_{\tau 1}+\tilde{s}_{12}^{e} v_{\tau 2} & \tilde{c}_{12}^{e} v_{\tau 2}+\tilde{s}_{12}^{e} e^{i \sigma} v_{\tau 1} & v_{\tau 3}
\end{array}\right)
$$

where with lower $v_{e 1}, v_{e 2}$ etc. are denoted the matrix elements of the leptonic mixing matrix $U_{e}^{\dagger}$. These forms of quark and leptonic mixing matrices are convenient since the contributions $v_{u s}, v_{u d}$ and $v_{e 1}, v_{e 2}$ etc. from the Yukawa matrices $\tilde{\mathbf{Y}}_{e, d}$ of (85), are separated from that arising from the initial 12-rotation $\tilde{O}_{12}^{d, e}$. Had the matrices $\tilde{\mathbf{Y}}_{e, d}$ the form in (13) (i.e. vanishing 22 entry) the above transformations $U_{e, d}$ would be pure rotations. Then the only source of CP violation would reside in the residual phase $\sigma$ in $P_{ \pm}$driven by the $(1,3),(3,1)$ entries. It is worth noticing that the $\mathrm{CP}$ violating phase appearing in the quark and leptonic mixing 
matrices differs by $\pi$ (cfr. (84)). This feature comes from the antisymmetry of the 12 block, while it does not depend on the initial antisymmetry in the 23 and 13 blocks.

The GUT scale Yukawa eigenvalues $Y_{u, c, t}, Y_{d, s, b}, Y_{e, \mu, \tau}, Y_{1,2,3}$, are linked to the physical fermion masses through the renormalization group equations (RGE). For moderate values of $\tan \beta=v_{u} / v_{d}$, one obtains at one-loop? (see e.g. [32]):

$$
\begin{array}{lll}
m_{u}=Y_{u} R_{u} R_{u}^{S M} \eta_{u} B_{t}^{3} v_{u}, & m_{d}=Y_{d} R_{d} R_{d}^{S M} \eta_{d} v_{d}, & m_{e}=Y_{e} R_{e} R_{e}^{S M} v_{d} \\
m_{c}=Y_{c} R_{u} R_{u}^{S M} \eta_{c} B_{t}^{3} v_{u}, & m_{s}=Y_{s} R_{d} R_{d}^{S M} \eta_{s} v_{d}, & m_{\mu}=Y_{\mu} R_{e} R_{e}^{S M} v_{d} \\
m_{t}=Y_{t} R_{u} R_{u}^{S M} B_{t}^{6} v_{u}, & m_{b}=Y_{b} R_{d} R_{d}^{S M} \eta_{b} B_{t} v_{d}, & m_{\tau}=Y_{\tau} R_{e} R_{e}^{S M} v_{d},
\end{array}
$$

and for the neutrino masses

$$
m_{1,2,3}=\frac{Y_{1,2,3}}{M} R_{\nu} R_{\nu}^{S M} B_{\tilde{t}}^{6} v_{2}^{2}
$$

where the factors $R_{u, d, e, \nu}$ and $R_{u, d, e, \nu}^{S M}$ account for the gauge-coupling induced running from the GUT scale $M_{G} \simeq 10^{16} \mathrm{GeV}$ to the SUSY breaking scale $M_{S} \simeq M_{t}$ and from $M_{S}$ to the electroweak scale $M_{Z}$, respectively. The factors $\eta_{f}$ encapsulate the QCD+QED running from $M_{S}$ down to $m_{f}$ for $f=b, c$ (or to $\mu=1 \mathrm{GeV}$ for the light quarks $f=u, d, s$ ). Namely, for $\alpha_{s}\left(M_{Z}\right)=0.119 \pm 0.004$ we have

$$
\begin{array}{llll}
R_{u} R_{u}^{S M}=3.53_{-0.07}^{+0.06}, & R_{d} R_{d}^{S M}=3.43_{-0.06}^{+0.07} & R_{e} R_{e}^{S M}=1.50, & R_{\nu} R_{\nu}^{S M}=1.15, \\
\eta_{b}=1.53_{-0.04}^{+0.03}, & \eta_{c}=2.05_{-0.11}^{+0.13}, & \eta_{u, d, s}=2.38_{-0.19}^{+0.24} .
\end{array}
$$

The factor $B_{t}$ includes the running induced by the large top quark Yukawa constant $\left(Y_{t} \sim\right.$ 1). . $^{2}$ For $Y_{t}$ varying from the lower limit $Y_{t}=0.5$, imposed by the top pole-mass, to the perturbativity limit $Y_{t} \approx 3$, the function $B_{t}$ decreases from 0.9 to 0.7 . Regarding the CKM elements, their physical values are related to the corresponding GUT-scale quantity (labelled by the superscript $G$ ) as follows 25 :

$$
\begin{aligned}
& V_{u s(d)}=V_{u s(d)}^{G}, \quad V_{c s(d)}=V_{c s(d)}^{G}, \quad V_{t b}=V_{t b}^{G}, \\
& V_{c(u) b}=V_{c(u) b}^{G} B_{t}^{-1}, \quad V_{t d(s)}=V_{t d(s)}^{G} B_{t}^{-1} .
\end{aligned}
$$

Using the procedure outlined in detail in [13], the following relations (valid at the GUT scale) are derived from the matrices (85):

$$
\begin{aligned}
& D=Y_{\tau}\left[\frac{1-\left(b+b^{-1}\right) \frac{Y_{\mu}-Y_{e}}{Y_{\tau}}}{F_{e}}\right]^{1 / 2}=Y_{b}\left[\frac{1-\left(b+b^{-1}\right) \frac{Y_{s}-Y_{d}}{Y_{b}}}{F_{d}}\right]^{1 / 2}, \\
& F_{e(d)}=1+\tilde{c}_{12}^{e(d) 2} Y_{c t}\left(b+b^{-1}\right) \cos \xi
\end{aligned}
$$

and

$$
A^{2} D=Y_{e} Y_{\mu} Y_{\tau}=k_{3}^{2} Y_{d} Y_{s} Y_{b}
$$

\footnotetext{
${ }^{23}$ The notation is the standard one: for the heavy quarks $t, b, c, m_{t, b, c}$ are their running masses respectively at $\mu=m_{t, b, c}$, while for the light quarks $m_{u, d_{s}}$ are given at $\mu=1 \mathrm{GeV}$.

${ }^{24}$ In the RG running of neutrino masses 90 ), the factor $B_{\tilde{t}}$ is function of $Y_{t} / \sin \beta$.

${ }^{25}$ We shall not take into account the analogous RGEs 33 for the neutrino mixings, since the experimental data still contain big error bars and such an improvement is not justified. Moreover, renormalization effects are mostly expected in case of strong mass degeneracy 34 which is not our case.
} 


$$
\tilde{B}^{2}=\frac{b}{\tilde{k}_{1}^{2}}\left(Y_{\mu}-Y_{e}\right) Y_{\tau} \frac{I_{e}}{F_{e}}=b\left(Y_{s}-Y_{d}\right) Y_{b} \frac{I_{d}}{F_{d}},
$$

where we have defined

$$
I_{e}=1+\tilde{c}_{12}^{e 2} \frac{Y_{\tau}}{Y_{\mu}-Y_{e}} Y_{c t} \cos \xi, \quad I_{d}=1+\tilde{c}_{12}^{d 2} \frac{Y_{b}}{Y_{s}-Y_{d}} Y_{c t} \cos \xi
$$

In the following we directly substitute the Yukawa constant ratios with the corresponding mass ratios whenever the latter are RGE invariant, e.g. $Y_{\mu} / Y_{\tau}=m_{\mu} / m_{\tau}, Y_{d} / Y_{s}=m_{d} / m_{s}$, etc. Now, by dividing the squared of the l.h.s and r.h.s in eq. (93) by the corresponding sides of eq. (96) and inverting, we obtain the following implicit relation:

$$
\frac{Y_{s}-Y_{d}}{Y_{b}}=\frac{I_{e} F_{d} \tilde{k}_{1}^{-2}}{I_{d} F_{e} Z^{2}} \frac{m_{\mu}-m_{e}}{m_{\tau}} \approx 0.059 \frac{I_{e} F_{d} \tilde{k}_{1}^{-2}}{I_{d} F_{e} Z^{2}}
$$

where

$$
Z=\left(\frac{F_{d}}{F_{e}}\right)^{1 / 2}\left[1-\left(1-\frac{I_{e}}{I_{d} \tilde{k}_{1}^{2}}\right)\left(b+b^{-1}\right) \frac{m_{\mu}-m_{e}}{m_{\tau}}\right]^{1 / 2} .
$$

Substituting the expression (98) back into eq. (93) we get:

$$
\frac{Y_{b}}{Y_{\tau}}=Z
$$

which shows the modification induced on the expression of the $b-\tau$ Yukawa unification at the GUT scale by the asymmetry in the 23 block. Analogously, by utilizing the relation (100) in eq. (96), the latter can be rewritten as

$$
\frac{Y_{s}-Y_{d}}{Y_{\mu}-Y_{e}}=\frac{\tilde{k}_{1}^{-2}}{Z} \frac{I_{e} F_{d}}{I_{d} F_{e}}
$$

while by dividing the squared of the r.h.s. and l.h.s. of (96) by the corresponding sides of (95) we have:

$$
\frac{m_{s}}{m_{d}}+\frac{m_{d}}{m_{s}}-2=\frac{k_{3}^{2}}{\tilde{k}_{1}^{4} Z}\left(\frac{I_{e} F_{d}}{I_{d} F_{e}}\right)^{2}\left(\frac{m_{\mu}}{m_{e}}+\frac{m_{e}}{m_{\mu}}-2\right) \approx 204.7 \frac{k_{3}^{2}}{\tilde{k}_{1}^{4} Z}\left(\frac{I_{e} F_{d}}{I_{d} F_{e}}\right)^{2} .
$$

Compare the present expressions (98, 101, 102) and (99) with the corresponding ones in eqs. (14,15) and (16) obtained in the Fritzsch-like case $\left(C=0, Y_{c t}, Y_{u t} \rightarrow 0\right.$, i.e. $\left.F_{e, d}, I_{e, d} \rightarrow 1\right)$. From eqs. (100) and (101) we can extract the following physical masses:

$$
\begin{aligned}
& m_{b}=\frac{R_{d} R_{d}^{S M} \eta_{b}}{R_{e} R_{e}^{S M}} B_{t} Z m_{\tau}=B_{t} Z \cdot\left(6.22_{-0.27}^{+0.25}\right) \mathrm{GeV} \\
& m_{s}-m_{d}=\frac{R_{d} R_{d}^{S M} \eta_{d}}{R_{e} R_{e}^{S M}} \frac{I_{e} F_{d}}{\tilde{k}_{1}^{2} Z I_{d} F_{e}}\left(m_{\mu}-m_{e}\right)=\frac{4 I_{e} F_{d}}{\tilde{k}_{1}^{2} Z I_{d} F_{e}} \cdot\left(143_{-14}^{+18}\right) \mathrm{MeV} .
\end{aligned}
$$

We now turn to the quark and lepton mixing. For the angles $\theta_{23}^{e, d}$ and the corresponding phases appearing in the unitary transformation (86) we find:

$$
\tan 2 \theta_{23}^{e}=2 \sqrt{b} \sqrt{\left(\frac{m_{\mu}-m_{e}}{m_{\tau}}\right)\left(1+\frac{2}{b} Y_{c t} \cos \xi\right)} \frac{\left[\left(1-I_{e}\left(b+b^{-1}\right) \frac{m_{\mu}-m_{e}}{m_{\tau}}\right)\right]^{1 / 2}}{1-2 b \frac{m_{\mu}-m_{e}}{m_{\tau}}+\left(b-b^{-1}\right) Y_{c t} \cos \xi},
$$




$$
\begin{aligned}
\tan 2 \theta_{23}^{d} & =\frac{2}{\sqrt{b}} \sqrt{\left(\frac{Y_{s}-Y_{d}}{Y_{b}}\right)\left(1+2 b Y_{c t} \cos \xi\right)} \frac{\left[\left(1-I_{d}\left(b+b^{-1}\right) \frac{Y_{s}-Y_{d}}{Y_{b}}\right)\right]^{1 / 2}}{1-2 b^{-1} \frac{Y_{s}-Y_{d}}{Y_{b}}+\left(b-b^{-1}\right) Y_{c t} \cos \xi}, \\
\tan \phi_{23}^{e} & =\frac{\frac{Y_{c t}}{b} \sin \xi}{1+\frac{Y_{c t}}{b} \cos \xi} \approx \frac{Y_{c t}}{b} \sin \xi \\
\tan \phi_{23}^{d} & =\frac{b Y_{c t} \sin \xi}{1+b Y_{c t} \cos \xi} \approx b Y_{c t} \sin \xi
\end{aligned}
$$

while the right rotation angles $\theta_{23}^{\prime e, d}$ and $\phi_{23}^{\prime e, d}$ are obtained from these expressions substituting respectively $b \rightarrow b^{-1}$. The phases appear strongly suppressed by the tiny $Y_{c t}$ parameter: for $Y_{c t} \sim 3 \cdot 10^{-3}$ and $b \sim 10, \phi_{23}^{d} \lesssim 0.01$. The 13 mixing angles are

$$
\begin{aligned}
& \sin \theta_{13}^{e}=\sqrt{a_{e}} \frac{s_{23}^{\prime e}}{c_{23}^{\prime e}} \frac{\sqrt{m_{e} m_{\mu}}}{m_{\tau}}=\left(\frac{m_{e} m_{\mu}^{2}}{b c_{23}^{e} m_{\tau}^{3}}\right)^{1 / 2} \leq 10^{-3}, \quad a_{e}=\frac{c_{23}^{\prime e}}{c_{23}^{e}} \\
& \frac{s_{13}^{d}}{s_{23}^{d}}=\frac{1}{\sqrt{a_{d}}} \frac{s_{23}^{\prime d}}{c_{23}^{\prime d} s_{23}^{d}} \sqrt{\frac{m_{d}}{m_{s}}} \frac{Y_{s}}{Y_{b}}, \quad a_{d}=\frac{c_{23}^{d}}{c_{23}^{\prime d}} .
\end{aligned}
$$

One can easily verify that for the corresponding phases $\phi_{13}^{e, d}=\phi_{23}^{\prime e, d}$. Finally the $U_{12}$ transformations are expressed by the following angles:

$$
\begin{aligned}
& \tan 2 \theta_{12}^{e}=2 \sqrt{a_{e}} \sqrt{\frac{m_{e}}{m_{\mu}}} \frac{\left[1-\left(a_{e}+a_{e}^{-1}\right) \frac{m_{e}}{m_{\mu}}\right]^{1 / 2}}{1-2 a_{e} \frac{m_{e}}{m_{\mu}}} \approx \frac{2}{\sqrt{c_{23}^{e}}} \sqrt{\frac{m_{e}}{m_{\mu}-m_{e}}} \approx \frac{0.140}{\sqrt{c_{23}^{e}}}, \\
& \tan 2 \theta_{12}^{d}=\frac{2}{\sqrt{a_{d}}} \sqrt{\frac{m_{d}}{m_{s}}} \frac{\left[1-\left(a_{d}+a_{d}^{-1}\right) \frac{m_{d}}{m_{s}}\right]^{1 / 2}}{1-2 a_{d}^{-1} \frac{m_{d}}{m_{s}}} \approx 2 \sqrt{c_{23}^{\prime d}} \sqrt{\frac{m_{d}}{m_{s}-m_{d}}} .
\end{aligned}
$$

while the corresponding phases are

$$
\begin{aligned}
\tan \phi_{12}^{e} & \approx \frac{\sin \xi}{Y_{c t}^{-1} \frac{m_{\mu}}{m_{\tau}}-\cos \xi} \lesssim\left[\left(Y_{c t}^{-1} \frac{m_{\mu}}{m_{\tau}}\right)^{2}-1\right]^{-1 / 2} \approx 4.6 \times 10^{-2} \\
\tan \phi_{12}^{d} & \approx \frac{\sin \xi}{Y_{c t}^{-1} \frac{Y_{s}}{Y_{b}}-\cos \xi} \approx \frac{\sin \xi}{\frac{Y_{c t}^{-1}}{\left(k_{1} Z\right)^{2}} \frac{m_{\mu}}{m_{\tau}}-\cos \xi} \lesssim\left[\left(Y_{c t}^{-1} \frac{m_{\mu}}{m_{\tau}}\right)^{2}\left(\frac{1}{k_{1} Z}\right)^{4}-1\right]^{-1 / 2}
\end{aligned}
$$

We can easily estimate that $\phi_{12}^{d}$ can be at most $11^{\circ}$ and $25^{\circ}$ with $b \gtrsim 1, \quad Y_{c t}=3 \times 10^{-3}$ and for $k_{1}=2$ and $k_{1}=-3$, respectively. Moreover, the larger is the asymmetry parameter $b$, the smaller the function $Z$ becomes and therefore the smaller the phase $\phi_{12}^{d}$ appears. In conclusion, the phase $\xi$ cannot provide by itself a sizeable source of $\mathrm{CP}$ violation since it is driven by the tiny ratio $Y_{c t}$. That is the reason motivating us to introduce non-vanishing $(1,3),(3,1)$ entries driving the extra phase $\sigma$. Hence, as a matter of fact we may think of the matrices $U_{e, d}$ as pure rotations, parameterised in the standard form (7) with the phase $\delta=\pi$ [13].

In the analysis, we test the amount of CP-violation through the parameter $\varepsilon_{K}$ describing the $\mathrm{CP}$ violation in $K \rightarrow \pi \pi$. More precisely, we use the more uncertain parameter $B_{K}$ 


\begin{tabular}{||c|c||}
\hline \hline Observables & Values \\
\hline$\bullet m_{e}[\mathrm{MeV}]$ & 0.511 \\
\hline$\bullet m_{\mu}[\mathrm{MeV}]$ & 105.7 \\
\hline$\bullet m_{\tau}[\mathrm{GeV}]$ & 1.777 \\
\hline$\bullet m_{u} / m_{t}$ & $(0.9 \div 3) \times 10^{-5}$ \\
\hline \hline$\bullet m_{c} / m_{t}$ & $(8.0 \pm 1.3) \times 10^{-3}$ \\
\hline$\bullet Q$ & $22.7 \pm 0.8$ \\
\hline$\bullet\left|\varepsilon_{K}\right|$ & $(2.280 \pm 0.013) \times 10^{-3}$ \\
\hline \hline$m_{s}[\mathrm{MeV}]$ & $155 \pm 75$ \\
\hline$m_{b}[\mathrm{GeV}]$ & $4.25 \pm 0.15$ \\
\hline$m_{s} / m_{d}$ & $21 \pm 4$ \\
\hline$m_{u} / m_{d}$ & $0.2 \div 0.7$ \\
\hline$M_{t}[\mathrm{GeV}]$ & $173.8 \pm 5.2$ \\
\hline$\left|V_{c b}\right|$ & $0.0395 \pm 0.0017$ \\
\hline$\left|V_{u s}\right|$ & $0.2196 \pm 0.0023$ \\
\hline$\left|V_{u b} / V_{c b}\right|$ & $0.093 \pm 0.014$ \\
\hline$\left|V_{t d}\right|$ & $0.0088 \pm 0.0018$ \\
\hline$\left|V_{t d} / V_{t s}\right|$ & $<0.22$ \\
\hline$B_{K}$ & $0.8 \pm 0.2$ \\
\hline \hline
\end{tabular}

Table 4: Physical quantities used in the phenomenological analysis. Those marked by $\bullet$ are used as input for the fits. The bounds on $\left|V_{t d}\right|$ and $\left|V_{t d} / V_{t s}\right|$ are inferred from the evaluation of $B_{d}^{0}-\bar{B}_{d}^{0}$ and $B_{s}^{0}-\bar{B}_{s}^{0}$ transitions in the standard model [9] and thus they are not literally valid in the context of supersymmetric model; even in the MSSM framework, with flavour-aligned soft terms, one can expect substantial supersymmetric contributions to these transitions.

parameterising the deviation from the vacuum saturation limit in $K^{0}-\bar{K}^{0}$ transition which we 'extract' from the expression of $\varepsilon_{K}$ as given in the standard model [35]:

$$
B_{K}=\left|\varepsilon_{K}\right|\left[\frac{f_{K}^{2} M_{K}}{\Delta M_{K}} \frac{G_{F}^{2} m_{W}^{2}}{12 \sqrt{2} \pi^{2}} \frac{\operatorname{Im}\left(\mathcal{F}^{\star} t_{u}^{2}\right)}{\left|t_{u}\right|^{2}}\right]^{-1} .
$$

and compare with its theoretical value which ranges from 0.6 to 1 . Here $f_{K}=161 \mathrm{MeV}$ is the kaon decay constant, $M_{K}=497.7 \mathrm{MeV}$ is the $K^{0}$ mass, and $\Delta M_{K}=0.53 \times 10^{10} \mathrm{~s}^{-1}$ is the experimental value of $K_{L}-K_{S}$ mass difference. The mixing angles enter through the combinations $t_{\alpha}=V_{\alpha s}^{\star} V_{\alpha d}: t_{u}$ appears explicitly, ${ }^{26}$ whereas $t_{c}, t_{t}$ are contained in the function

$$
\mathcal{F}=\eta_{1} t_{c}^{2} S_{0}\left(x_{c}\right)+\eta_{2} t_{t}^{2} S_{0}\left(x_{t}\right)+2 \eta_{3} t_{c} t_{t} S_{0}\left(x_{c}, x_{t}\right)
$$

where $\eta_{1} \sim 1.38, \eta_{2} \sim 0.57, \eta_{3} \sim 0.47$ are QCD correction factors and $S_{0}$ is one of the InamiLim functions $\left(x_{c, t}=\frac{m_{c, t}^{2}}{m_{W}^{2}}\right)$. In this way, we are testing whether the experimental value of $\varepsilon_{K}$ can be reproduced entirely by the standard model contribution associated with the box

\footnotetext{
${ }^{26}$ Note that in most of $V_{C K M}$ parameterizations, the factor $t_{u}$, coming from the amplitude of the decay $K^{0} \rightarrow 2 \pi$, is made real and thereby the factor $t_{u}^{2} /\left|t_{u}^{2}\right|$ becomes 1 . In our final parametrization of the CKM matrix this is not the case - see (87).
} 
diagrams involving the charm, top quark and the W boson. It is known, that in the MSSM or more generally, in models with flavour-aligned soft terms like in [36], the supersymmetric contributions are negligible. However, in general SUSY GUT context $\varepsilon_{K}$ can receive substantial contributions from the super-partners at the weak scale [37, 38]. In view of this, we shall not use the information from $\varepsilon_{K}$ to constrain the parameter space.

\subsection{Model parameters versus physical observables}

We can now proceed by confronting the parameters with the physical observables described by the model. The number of the former amount to 14 , namely $Y_{u, c, t}, A, B, D, \tilde{t}_{12}^{d}, b, \sigma, \xi, k_{1}, k_{2}$, $k_{3}, M_{L}$ while the number of observables is 20: the up-quark masses $m_{u, c, t}$, the down-quark masses $m_{d, s, b}$, the charged-lepton masses $m_{e, \mu, \tau}$, the neutrino masses $m_{1,2,3}$, four independent CKM parameters and other four parameters of the leptonic mixing matrix. As it will be discussed more extensively below, the three Clebsch coefficients $k_{1}, k_{2}, k_{3}$ are treated as external parameters, fixed at some specific GUT-inspired value.

The various physical observables deserve a different treatment in the analysis for at least two reasons. First, they are known with different degree of accuracy. Second, some of them have a minor impact on the determination of the 'flavour' parameters themselves. Therefore, the observables are classified and used as follows:

1. $m_{e}, m_{\mu}, m_{\tau}$ : these masses are determined with high accuracy so they are used as inputs to extract the parameters $A, B, D$ via eqs. (93966). Notice that in this way, also the explicit dependence on $\tan \beta$ of the fermion masses is automatically absorbed in the parameters $A, B, D$.

2. $\frac{m_{u}}{m_{t}}, \frac{m_{c}}{m_{t}}$ : these mass ratios enter only into the determination of $Y_{u t}, Y_{c t}$ and are used as inputs ${ }^{27}$. Notice, however, that $Y_{u t}, Y_{c t}$ quite depend on $Y_{t}$ through the renormalization factor $B_{t}$ : e.g. $Y_{c t}=m_{c} / m_{t} B_{t}^{3} \eta_{c}^{-1}$. As this ratio is tiny, the dependence on $m_{c} / m_{t}$ is weak and hence our results are not sensitive to the corresponding errors on $m_{c}$ and $m_{t}$. This justifies the fact that we use the central values of the ratio $m_{c} / m_{t}$ shown in Table 6. On the other hand, the Yukawa constant $Y_{t}$ is left as free parameters. Indeed, it is not sensible to fix $Y_{t}$ by the top mass due to its infrared behaviour. It will turn out that (in almost every case) the preferred value for $Y_{t}$ is 0.5 which enhances the effect of $Y_{c t}$. This value is indeed the smallest one compatible with the top mass for which $m_{t}=(160.9 \pm 2.2) \sin \beta \mathrm{GeV}$ for $\alpha_{s}\left(M_{z}\right)=0.119 \pm 0.004$. Correspondingly, we have for the ratio $Y_{c t}=\left(2.7_{+0.2}^{-0.1}\right) \cdot 10^{-3}$.

3. $m_{d}, m_{s}, m_{b},\left|V_{u s}\right|,\left|V_{c b}\right|,\left|V_{u b} / V_{c b}\right|:$ these observables are fitted by a $\chi^{2}$-like procedure (see Sect. 4.4) to constrain the remaining parameters they depend on, namely $Y_{t}, b, \tilde{t}_{12}^{d}, \sigma, \xi$ once the Clebsch factors $k_{1}, k_{3}$ are prescribed (see Sect. 4.3).

4. $M_{t}, m_{u} / m_{d}$ : the top pole mass $M_{t}=m_{t} \cdot\left(1+\frac{4}{3 \pi} \alpha_{s}\left(M_{t}\right)+\frac{11.4}{\pi^{2}} \alpha_{s}^{2}\left(M_{t}\right)\right)$ is determined as output once $Y_{t}$ is fixed by the fit in point 3., modulo $\sin \beta$. Though in our analysis we cannot determine univocally (and in a sense is not necessary) $\tan \beta$, the model we presented in Sect. 3 naturally yields $\tan \beta$ in the small or moderate regime. Using the

\footnotetext{
${ }^{27}$ Now for the sake of discussion we consider also $Y_{u t}$ but in practice, as already mentioned, we neglect it at all.
} 
determination of $m_{s} / m_{d}$ from the fit, we extract the ratio $m_{u} / m_{d}$ from the combination 39

$$
Q=\frac{m_{s} / m_{d}}{\sqrt{1-\left(m_{u} / m_{d}\right)^{2}}}
$$

5. $B_{K}$ : the $\mathrm{CP}$ violation parameter is given as a prediction from the result of the fit performed in point 3 .

6. $\left|V_{\mu 3}\right|,\left|V_{e 2}\right|,\left|V_{e 3}\right| \&$ lepton CP-phase: these are also given as predictions. More precisely, for the sake of comparison with the experimental data, we trade these three elements for the corresponding 'oscillation' parameters: $\sin ^{2} 2 \theta_{23}^{l}=4\left|V_{\mu 3}\right|^{2}\left(1-\left|V_{\mu 3}\right|^{2}\right), \sin ^{2} 2 \theta_{12}^{l}=$ $4\left|V_{e 2}\right|^{2}\left(1-\left|V_{e 2}\right|^{2}\right)$ and $\sin ^{2} 2 \theta_{13}^{l}=4\left|V_{e 3}\right|^{2}\left(1-\left|V_{e 3}\right|^{2}\right)$. Notice, however, that the predictions for both $\mathrm{CP}$ violation phase and the element $\left|V_{e 2}\right|$ depend also on $\tilde{t}_{12}^{e}=\frac{k_{2}}{k_{1}} \tilde{t}_{12}^{d}$ i.e. on the Clebsch $k_{2}$.

7. $m_{1}, m_{2}, m_{3}$ : the neutrino masses follow immediately as predictions $m_{2} / m_{3}=b Y_{c t}$, $m_{1} / m_{2}=Y_{u c}$. However, the determination of any single mass does require the last parameter i.e. the mass scale $M_{L}=\frac{M}{\eta}$.

In summary, we have that from the sets 1) and 2) five parameters are fixed - $A, B, D, Y_{u t}, Y_{c t}$. From the set 3 ) other five parameters are constrained by the fit $-b, \tilde{t}_{12}^{d}, \sigma, \xi, Y_{t}$ once the two Clebsches $k_{1}, k_{3}$ have been assigned. The determination of these twelve parameters allows us to make five clean predictions: $m_{1} / m_{2}, m_{2} / m_{3}, B_{K},\left|V_{\mu 3}\right|,\left|V_{e 3}\right|$. Also $M_{t}$ is given as a prediction within the uncertainty of $\tan \beta$ expected to be in the range $1-10$. From the result of the fit, we also gain the ratio $m_{u} / m_{d}$ by using the information on the parameter $Q$ (fixed at its central value).

Finally, the neutrino masses depend on $M_{L}$ and both $\left|V_{e 2}\right|$ and the amount of CP violation in the lepton mixing matrix depend on the Clebsch coefficient $k_{2}$. For definiteness, the flavour mass scale $M$ and the lepton-violation mass scale $M_{L}$ are set at the GUT scale $M_{G}$, i.e. $M=$ $M_{L}=M_{G}$ which as already discussed means that $\frac{b}{\eta Y_{t}} \sim 10^{-1}$ or $\eta \sim 10^{2}$ for $Y_{t} \sim 1, b \sim 10$.

In the next Section we shall discuss more extensively how we handle the Clebsch factors.

Finally, the phenomenological analysis requires also the parameters associated with the standard model gauge sector: $\alpha_{s}\left(M_{Z}\right), \alpha_{e m}, \sin ^{2} \theta_{w}$. For $\alpha_{e m}, \sin ^{2} \theta_{w}$ we use the central values quoted in [9]. As regards $\alpha_{s}\left(M_{Z}\right)$, the present world average is $\alpha_{s}\left(M_{Z}\right)=0.119 \pm 0.002$ [9]. On the other hand the $S U(5)$ unification of gauge couplings implies a larger value, $\alpha_{s}\left(M_{Z}\right) \approx 0.125$. Therefore, as a compromise, we have taken $\alpha_{s}\left(M_{Z}\right)=0.123$. We remind that only $m_{b}$ and $m_{s}$ are sensitive to variations of $\alpha_{s}\left(M_{Z}\right)$ within the quoted error. The corresponding variations can be inferred from the gauge-renormalization factors given in (91). On the other hand, the $\alpha_{s}\left(M_{Z}\right)$ dependence of the running factor $B_{t}$ is less than $1 \%$ and therefore all other observables are not sensitive to $\alpha_{s}\left(M_{Z}\right)$.

\subsection{Clebsch prescription: three different ansätze}

First let us recall how the Clebsch coefficients enter into the determination of the physical quantities. The Yukawa eigenvalues $Y_{e, \mu, \tau}$ and $Y_{d, s, b}$ depend only on $k_{1}$ and $k_{3}$ (see the analytical expressions in Sect. 4.1) Similarly, the mixing angles $v_{u s}, v_{u d}, \cdots$ also depend only on

\footnotetext{
${ }^{28}$ The dependence on $k_{2}$ contained in $\tilde{k}_{1}$ in eq. (85) is mild for $\tilde{t}_{12}^{d}<0.1$.
} 
$k_{1}, k_{3}$. The coefficient $k_{2}$ affects only the lepton mixing matrix (88) via the initial 12 rotations $\tilde{t}_{12}^{e}=\frac{k_{2}}{k_{1}} \tilde{t}_{12}^{d}$. Thereby $k_{2}$ can only be fixed for example by the 12 lepton mixing angle $V_{e 2}$ through the SN parameter $\sin ^{2} 2 \theta_{12}^{l}=4\left|V_{e 2}\right|^{2}\left(1-\left|V_{e 2}\right|^{2}\right)$. Notice, indeed, that both $\sin ^{2} 2 \theta_{13}^{l}$ and $\sin ^{2} 2 \theta_{23}^{l}$ do not depend on $k_{2}$.

We can guess very easily the needed range for such $k_{1,2,3}$. Indeed $k_{1,3}$ are mainly fixed by the strange mass in (103) and the ratio $m_{s} / m_{d}$ in (102). From $m_{s}$ we infer that $k_{1}$ is to lie within $\frac{5}{3} \div 3$ - for $b \gtrsim 8$. Correspondingly, from $m_{s} / m_{d}, k_{3}$ is forced to be in the range $1 \div \frac{8}{3}$. Hence, we have performed a preliminary scanning of the parameter space, by fitting the quark observables in point 3 (Sect. 4.2 ) with $k_{1}, k_{3}$ free to float in the range $0 \div 3$. In such a way we have specified three Clebsch prescriptions for $k_{1}$ and $k_{3}$ and they are given in eqs. (59, 60) and (61), featuring three different ansätze $\mathbf{A}, \mathbf{B}, \mathbf{C}$, recalled here for convenience:

$$
\begin{array}{ll}
\text { ansatz A: } & k_{1}=2, \quad k_{3}=1 \\
\text { ansatz B: } & k_{1}=k_{3}=-3, \\
\text { ansatz C: } & k_{1}=-3, \quad k_{3}=2
\end{array}
$$

On the other hand a priori we cannot stick to any specific value of $k_{2}$ : only the result of the fit will allow us to envisage the value that can give the better prediction for $\sin ^{2} 2 \theta_{12}^{l}$. For that it is useful to write down explicitly² $V_{e 2}$ :

$$
\left|V_{e 2}\right|^{2}=\tilde{c}_{12}^{e 2} v_{e 2}^{2}+\tilde{s}_{12}^{e 2} v_{e 1}^{2}+2 \tilde{c}_{12}^{e} \tilde{s}_{12}^{e} v_{e 2} v_{e 1} \cos \sigma
$$

The present MSW range in eq. (2) translates into $\left|V_{e 2}\right|=(1.6-6) \cdot 10^{-2}$. The lower elements of $U_{e}^{\dagger}$ read as

$$
v_{e 2} \approx-s_{12}^{e} c_{23}^{e}, \quad v_{e 1} \approx c_{12}^{e} c_{13}^{e} \sim 1
$$

then from eqs. (110) we have $\left|v_{e 2}\right| \approx \sqrt{\frac{c_{23}^{e} m_{e}}{m_{\mu}}} \sim 0.07 \sqrt{c_{23}^{e}}$ which slowly decreases with the asymmetry parameter $b-$ for $b \gtrsim 8$ it becomes around $(5-6) \cdot 10^{-2}$ saturating by itself the present experimental upper limit. Therefore, the r.h.s. of eq.(117) tells us that the third term can reduce $\left|V_{e 2}\right|$ if $\tilde{t}_{12}^{e} \lesssim 0.1$ and $\sigma<90^{\circ}$. In this way $\sin ^{2} 2 \theta_{12}^{l}$ may become smaller than $10^{-2}$. Interestingly, it will turn out that the quark-sector fit demands the corresponding initial 12 rotation $\tilde{t}_{12}^{d}$ to lie in a similar range, $\tilde{t}_{12}^{d} \lesssim 0.1$, in all the three ansätze. We also write the CKM elements in (87) to explicitly show the interplay between the 12 rotation $\tilde{t}_{12}^{d}$ and the CP-phase $\sigma$ :

$$
\begin{aligned}
& \left|V_{u s}\right|^{2}=\tilde{c}_{12}^{d 2} v_{u s}^{2}+\tilde{s}_{12}^{d 2} v_{c s}^{2}+2 \tilde{c}_{12}^{d} \tilde{s}_{12}^{d} v_{u s} v_{c s} \cos \sigma \\
& \left|V_{c b}\right|^{2}=\tilde{c}_{12}^{d 2} v_{c b}^{2}+\tilde{s}_{12}^{d 2} v_{u b}^{2}-2 \tilde{c}_{12}^{d} \tilde{s}_{12}^{d} v_{c b} v_{u b} \cos \sigma \\
& \left|V_{u b}\right|^{2}=\tilde{c}_{12}^{d 2} v_{u b}^{2}+\tilde{s}_{12}^{d 2} v_{c b}^{2}+2 \tilde{c}_{12}^{d} \tilde{s}_{12}^{d} v_{c b} v_{u b} \cos \sigma
\end{aligned}
$$

where

$$
v_{u s}=c_{13}^{d} s_{12}^{d} \approx s_{12}^{d}, \quad v_{c s}=c_{12}^{d} c_{23}^{d} \sim 1 \quad v_{c b} \approx s_{23}^{d}, \quad v_{u b}=-s_{13}^{d} .
$$

Therefore, if the fit of the quark quantities selects $\sigma<90^{\circ}$ and $\tilde{t}_{12}^{d} \lesssim 0.1$, then the choice $k_{2}=k_{1}$ is favoured as it implies $\tilde{t}_{12}^{e}=\tilde{t}_{12}^{d}$. On the other hand, if it comes out that $\sigma \geq 90^{\circ}$

\footnotetext{
${ }^{29}$ For the sake of brevity we are assuming that the elements of the mixing matrices $U_{e, d}$ are real as we have seen that the phases $\phi_{i j}^{e, d}$ are typically tiny.
} 
(and irrespectively of $\tilde{t}_{12}^{d}$ ) then $k_{2}=0$ (i.e. $\tilde{t}_{12}^{e}=0$ ) is preferable in order not to raise $\sin ^{2} 2 \theta_{12}^{l}$ above $1.4 \cdot 10^{-2}$. As we shall see in the next section the three ansätze will prefer different values of the phase $\sigma$ such that in the ansatz $\mathbf{A} k_{2}=k_{1}=2$ can be chosen and as a result $\sin ^{2} 2 \theta_{12}^{l}<0.01$ can be obtained. On the other hand, in both the other scenarios we have to select $k_{2}=0$ and content ourselves with $\sin ^{2} 2 \theta_{12}^{l}>0.01$. Needless to say that this picture in which all the Clebsch $k_{1,2,3}$ are taken real is "minimal". For example, by allowing for complex $k_{n}$, the lepton and quark mixing matrices would have different $\mathrm{CP}$ violating phases and therefore, for example, also in the ansätze $\mathbf{B}, \mathbf{C}$ we could achieve $\sin ^{2} 2 \theta_{12}^{l}<0.01$ with non-vanishing $\tilde{t}_{12}^{e}$. Finally, one may wonder about the alternative possibility to account for the large-mixing angle MSW solution of the SN anomaly requiring $\sin ^{2} 2 \theta_{12}^{l} \sim 0.5-0.98$. That parameter range translates into $\left|V_{e 2}\right|>0.4$ which could be achieved within our ansätze by large $\tilde{t}_{12}^{e}-\tilde{t}_{12}^{e} \gtrsim 0.7-0.8$, or $\frac{k_{2}}{k_{1}} \gtrsim 7-8$. Such $O(10)$ Clebsch coefficients seem to come less naturally and probably at the price of a less economical Higgs content.

\subsection{Strategy for the fit}

To study the constraints on the parameters of the model from the down-quark masses and the CKM mixing angles we have performed an alternative $\chi^{2}-$ analysis. 00

The CKM elements $\left|V_{u s}\right|,\left|V_{c b}\right|,\left|V_{u b} / V_{c b}\right|$ are assumed to be mainly affected by Gaussian errors and therefore the standard $\chi^{2}$-function can be assumed for them:

$$
\chi^{2}=\sum_{a}\left(\frac{\Delta_{a}}{\sigma_{a}}\right)^{2}, \quad \Delta_{a}=x_{a}-x_{a}^{e x p}
$$

where the theoretical outcome for a certain observable is denoted by $x_{a}$ and the corresponding experimental value and statistical error by $x_{a}^{e x p}$ and $\sigma_{a}$, respectively (see Table (1). On the contrary, the uncertainties affecting the determination of the down-quark masses are due to the dependence on the theoretical-model used to extract the masses from the measurements. For this reason we think that it is not correct to assign them a Gaussian distribution and hence to define the canonical $\chi^{2}$ - function. Nevertheless, we interpret the 1- $\sigma$ range reported in the Table 4 as a 'reasonable' interval accounting for all possible (different) determinations. Therefore, we prescribe the masses a flat distribution in that range. More precisely, we define the following $\chi^{2}$-like function for the masses:

$$
\begin{aligned}
& \tilde{\chi}_{b}^{2}=\left\{\begin{array}{ll}
0 & \text { if }\left|\Delta_{b}\right|<0.15 \\
\left(\frac{\left|\Delta_{b}\right|-0.15}{0.075}\right)^{2} & \text { if }\left|\Delta_{b}\right| \geq 0.15
\end{array},\right. \\
& \tilde{\chi}_{s}^{2}=\left\{\begin{array}{ll}
0 & \text { if }\left|\Delta_{s}\right|<75 \\
\left(\frac{m_{s}-80}{10}\right)^{4} & \text { if } \Delta_{s} \leq-75 \\
\left(\frac{m_{s}-230}{30}\right)^{4} & \text { if } \Delta_{s} \geq 75
\end{array},\right. \\
& \tilde{\chi}_{s / d}^{2}=\left\{\begin{array}{ll}
0 & \text { if }\left|\Delta_{s / d}\right|<3 \\
\left(\left|\Delta_{s / d}\right|-3\right)^{4} & \text { if }\left|\Delta_{s / d}\right| \geq 3
\end{array},\right.
\end{aligned}
$$

\footnotetext{
${ }^{30}$ Those observables are computed by numerically diagonalizing the Yukawa matrices (85) once the parameters $A, B, D$ are determined from the charged-lepton masses (for given $k_{1,2,3}$ ) through eqs. (93 96 ). The agreement between the numerical and the analitycal out-comings - presented in Sect. 4.1 - is at the level of one per mil.
} 


\begin{tabular}{||c||c|c|c|c||}
\hline \hline $\begin{array}{c}\text { Ansatz A: } \\
k_{1,2}=2, k_{3}=1\end{array}$ & $\begin{array}{c}I . C=0 \\
\left(Y_{t}, b, \xi\right)\end{array}$ & $\begin{array}{c}I I . Y_{c t}=0 \\
\left(Y_{t}, b, \sigma, \tilde{t}_{12}^{d}\right)\end{array}$ & $\begin{array}{c}I I I . \text { Complete } \\
\left(Y_{t}, b, \xi, \sigma, \tilde{t}_{12}^{d}\right)\end{array}$ & $\begin{array}{c}I V . \text { Spont.CP } \\
\left(Y_{t}, b, \tilde{t}_{12}^{d}\right)\end{array}$ \\
\hline \hline$m_{b}[\mathrm{GeV}]$ & 4.35 & 4.00 & 4.08 & 4.04 \\
\hline$m_{s}[\mathrm{MeV}]$ & 219 & 249 & 253 & 254 \\
\hline$m_{s} / m_{d}$ & 17.5 & 20.8 & 21.9 & 22.0 \\
\hline$\left|V_{u s}\right|$ & 0.2138 & 0.2197 & 0.2193 & 0.2198 \\
\hline$\left|V_{c b}\right|$ & 0.0507 & 0.0447 & 0.0437 & 0.0433 \\
\hline$\left|V_{u b} / V_{c b}\right|$ & 0.080 & 0.097 & 0.094 & 0.098 \\
\hline$\star\left|V_{t d}\right|$ & 0.0148 & 0.0140 & 0.0133 & 0.0133 \\
\hline$\star\left|V_{t d} / V_{t s}\right|$ & 0.30 & 0.33 & 0.32 & 0.32 \\
\hline$\star \mathrm{B}_{k}$ & $\infty$ & 1.9 & 0.84 & 0.77 \\
\hline$\star \sin ^{2} 2 \theta_{23}^{l}$ & 0.96 & 0.83 & 0.87 & 0.85 \\
\hline$\star \sin ^{2} 2 \theta_{12}^{l}$ & $1.2 \cdot 10^{-2}$ & $2.0 \cdot 10^{-3}$ & $5.2 \cdot 10^{-3}$ & $5.7 \cdot 10^{-3}$ \\
\hline$\star \sin ^{2} 2 \theta_{13}^{l}$ & $1.8 \cdot 10^{-2}$ & $2.6 \cdot 10^{-2}$ & $2.4 \cdot 10^{-2}$ & $2.4 \cdot 10^{-2}$ \\
\hline$\star m_{u} / m_{d}$ & 0.6 & 0.4 & 0.3 & 0.3 \\
\hline$\star M_{t} / \sin \beta$ & 172.7 & 172.7 & 172.7 & 172.7 \\
\hline \hline$Y_{t}$ & 0.5 & 0.5 & 0.5 & 0.5 \\
\hline$b$ & 10.0 & 12.1 & 11.8 & 12.0 \\
\hline$\xi$ & 0 & - & $180^{\circ}$ & $\pi$ \\
\hline$\sigma$ & - & $19.7^{\circ}$ & $43.4^{\circ}$ & $\pi / 4$ \\
\hline \hline$\tilde{t}_{12}^{d}$ & - & $3.4 \cdot 10^{-2}$ & $4.4 \cdot 10^{-2}$ & $4.7 \cdot 10^{-2}$ \\
\hline \hline$\tilde{\chi}_{\min }^{2} \& \chi_{\min }^{2}$ & $0.06 \& 50.7$ & $2.1 \& 9.5$ & $0.4 \& 6$ & $1 \& 5.1$ \\
\hline \hline & & & & \\
\hline & & & & \\
\hline
\end{tabular}

Table 5: The analysis of the ansatz $\mathbf{A}$. Taking as reference eq. (83) for $\mathbf{Y}_{e, d}$, the columns refer to: I. $(1,3)$ and $(3,1)$ entries are set to zero $(C=0)$, i.e. three-parameter fit $Y_{t}, b, \xi ; I I .(2,2)$ entry is set to zero $\left(Y_{c t} \rightarrow 0\right)$, i.e. four-parameter fit $Y_{t}, b, \sigma, \tilde{t}_{12}^{d} ; I I I$. complete pattern - five-parameter fit $Y_{t}, b, \xi, \sigma, \tilde{t}_{12}^{d}$. In all cases, from $Y_{t}=0.5$ it comes out $Y_{c t}=2.6 \cdot 10^{-3}$. The quantities marked by the symbol $\star$, are not included in the fits but are predictions of the fit itself in correspondence of best-fit parameters (also shown). The values at the minima of the (non-Gaussian) $\chi^{2}$-like function $\tilde{\chi}_{\min }^{2}$ (see eq. (123)) and of the Gaussian function, $\chi_{\min }^{2}$ are also given.

where $\tilde{\chi}_{b}^{2}, \tilde{\chi}_{s}^{2}, \tilde{\chi}_{s / d}^{2}$ refer to $m_{b}, m_{s}, m_{s} / m_{d}$, respectively and $\Delta_{b}, \Delta_{s}, \Delta_{s / d}$ are the corresponding deviations defined as in eq. (122). We can note from (123) that mass values outside the 1- $\sigma$ range are strongly penalized by the higher-power dependence of the (arbitrary) assigned distribution. The sum of the above functions will be denoted as $\tilde{\chi}^{2}=\tilde{\chi}_{b}^{2}+\tilde{\chi}_{s}^{2}+\tilde{\chi}_{s / d}^{2}$.

By minimizing the sum $\tilde{\chi}^{2}+\chi^{2}$, we rather test the consistency between the model and the Gaussian-distributed data within a reasonable range for the down-quark masses. In the next we shall report both the value of $\tilde{\chi}_{\min }^{2}$ and $\chi_{\min }^{2}$ separately, in Tables 5, 6 and 17 containing all the results of the fits. We have to remark that descriptions of the quark masses outside the 'reasonable' range may be accounted, for example, by uncertainties in $\alpha_{s}\left(M_{Z}\right)$. 


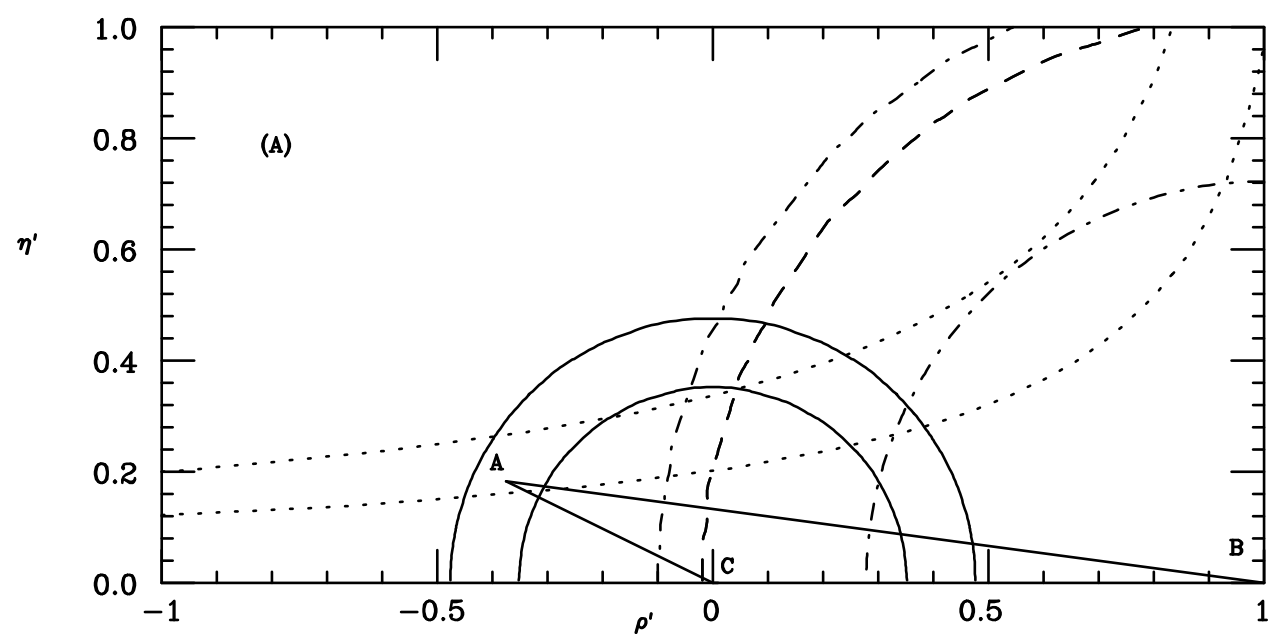

Figure 5: The unitarity triangle as emerging from the fit of the ansatz $\mathbf{A}$ (case $I I I)$ in the $\left(\rho^{\prime}, \eta^{\prime}\right)$ plane. The angles defined in eqs. (124) are to be identified as $\alpha \equiv C \hat{A} B, \beta \equiv A \hat{B} C, \gamma \equiv B \hat{C} A$. The parameters $\lambda, A$ are accordingly fixed as $\lambda=0.2193$ and $A=0.909$. We have drawn the 1- $\sigma$ contours of $R_{b}=0.414 \pm 0.062$ (solid lines) $R_{t}=0.913 \pm 0.191$ - as inferred from $\Delta M_{d}$ - (dot-dashed lines) and $R_{t}<1.02$ - from the lower bound on $\Delta M_{s}$ (dashed-line). The band enclosed by the hyperbola (dotted) refers to $B_{K}=0.8 \pm 0.2$.

\subsection{Testing theoretical models: fit and predictions}

We have found it to be instructive to consider first the case with $(1,3),(3,1)$ entries set to zero $(C=0)$, to investigate the effect of a non-zero 22 entry on the pattern (13). This pattern (denoted by $I$ ) has three parameters, $b, \xi$ and $Y_{t}$. As a second step, we have restored the $(1,3)(3,1)$ entries and set to zero the 22-entry (formally, $Y_{c t}=0$ ) leaving as free parameters $b, \tilde{t}_{12}^{d}, \sigma, Y_{t}$ (case denoted by $I I$ ).

Finally, the complete pattern is analysed with five free parameters, $b, \xi, \tilde{t}_{12}^{d}, \sigma, Y_{t}$ (case denoted by $I I I)$. We can first make some general considerations. We can note that the dependence on $Y_{c t}$ of the physical quantities is mainly encoded in the factors $I_{e}, I_{d}$. The former can be easily estimated, $I_{e} \approx 1+0.044 \cdot \cos \xi$ and thus $Y_{c t}$ can induce quite a small correction on the leptonic mixing angles. Therefore, from the expressions (104), we can infer that $\left|V_{\mu 3}\right| \equiv\left|v_{\mu 3}\right| \approx s_{23}^{e}$ (see (88)) depends mainly on the asymmetry parameter $b$, increasing roughly as $\sqrt{b}$. As a result, the AN bound $\sin ^{2} 2 \theta_{23}^{l}=4\left|V_{\mu 3}\right|^{2}\left(1-\left|V_{\mu 3}\right|^{2}\right)>0.8$ requires $6<b<$ 12 as it was found in the case (13) [13]. Analogously, in the case $I$ it is $\left|V_{e 2}\right| \equiv\left|v_{e 2}\right| \approx s_{12}^{e} c_{23}^{e}$ which weakly decreases with $b$. Then the MSW range, in the case $I$ can be recovered for $b \gtrsim 7$ where $\sin ^{2} 2 \theta_{12}^{l}=4\left|v_{e 2}\right|^{2}\left(1-\left|v_{e 2}\right|^{2}\right)$ gets smaller than $1.5 \cdot 10^{-2}$. As we already know, this mixing can be affected by the initial 12 rotation $\tilde{t}_{12}^{e}$ present in the full model (case $I I I$ ).

The discussion about $I_{d}$ affecting the quark observables is more involved as it depends, through the ratio $Y_{b} /\left(Y_{s}-Y_{d}\right) \propto k_{1}^{2}$, on the specific ansatz considered. However, in the case A a rough estimate gives $I_{d} \sim 1+0.12 \cos \xi$, while larger correction can be achieved in the ansätze $\mathbf{B}, \mathbf{C}, I_{d} \sim 1+0.25 \cos \xi$. In the following we discuss separately our results for the three ansätze, reported in Tables 5 , 6 and 0.

Ansatz A In the case $I$ (Table 5-second column), the result of the fit is acceptable though, due to the high accuracy achieved in the determination of the CKM angles, we can observe some discrepancy in the Cabibbo angle $\left|V_{u s}\right| \equiv\left|v_{u s}\right| \approx s_{12}^{d} \propto I_{d} / I_{e}$ and $\left|V_{c b}\right| \approx s_{23}^{d}$. The former appears to be quite small while the latter is somehow too large. These two quantities require in turn $\xi=0$ (to maximize $I_{d}$ ) and quite a large 23 asymmetry, $b=10$ (to minimize $s_{23}^{d}$ ) as 
best fit points. Notice that $b>10$, though preferable to further reduce $\left|V_{c b}\right|$, is not tolerated by $\left|V_{u s}\right|$ which would further decrease.

On the other hand, the quark masses fall in their 'reasonable' range. Noticeably, $Y_{t}=0.5$ is preferred in all the fits and as a result the prediction $M_{t} / \sin \beta \leq 172.7 \mathrm{GeV}$ turns out to be consistent. Interestingly, the predictions (marked by $\star$ ) for the leptonic mixing angle are quite good. We have to recall that whenever the quark mixing are 'reasonably' accommodated, the leptonic mixing angles fall automatically into the presently most favoured range thanks to the remarkable product rule (12) inherent in the theoretic structure of the model. For example the AN oscillatory mixing $\sin ^{2} 2 \theta_{23}^{l}$ is almost maximal, as the large $b=10$ implies. The 13 neutrino mixing remain well below the upper bound shown in Table 1 and $\sin ^{2} 2 \theta_{13}^{l}$ lies in the range $(1 \div 3) \cdot 10^{-2}$ in all the ansätze, as we will see (cfr. Tables 6, , 0). This range in the case of $\delta m_{23}^{2}$ close to the upper bound in (1), can be of interest for the experimental search of $\nu_{e} \rightarrow \nu_{\tau}$ oscillation in the future CERN Neutrino Factory [40].

We do not show in the tables the prediction for the neutrino mass ratios. However, as regards $m_{1} / m_{2}$ the prediction is just $m_{1} / m_{2}=Y_{u} / Y_{c}$ (in any of the ansätze) and so it is typically about $\sim 3 \cdot 10^{-3}$. On the other hand, the prediction for the ratio $m_{2} / m_{3}$ depends on the value of the asymmetry parameter $b^{\prime}$. In the simplest case $b^{\prime}=b$, it can be easily computed as $m_{2} / m_{3}=b Y_{c t}$ and so it is in the range $\sim(2-3) \cdot 10^{-2}$ in agreement with the present experimental hint. This out-coming will be similar in all the ansätze and it is a byproduct of the link between the neutrino and the up-quark masses and the large 23-asymmetry $b$ demanded by the quark phenomenology.

The other predictions, $V_{t d}, V_{t d} / V_{t s}$ are marginally compatible with the experimental values. We have also reported the expected mass ratio $m_{u} / m_{d}$ extracted from the 'ellipse' parameter $Q$ (116) which appears to be consistent with the determination of $m_{s} / m_{d}$ obtained from the fit.

Clearly there is no CP violation in the CKM matrix as the infinite value of $B_{K}$ does reflect. The situation gets improved in the case $I I$. The presence of the $(1,3),(3,1)$ entries introduces the initial 12 rotation $\tilde{t}_{12}^{d}$ and the phase $\sigma$ which strongly modifies the CKM elements (87). From the expressions of the Cabibbo angle in (119) and from the fact that the element $v_{u s}$ itself is around 0.2 , we can deduce that $\tilde{O}_{12}^{d}$ is to be a small rotation, $\tilde{t}_{12}^{d} \lesssim 0.1$, to prevent too large a correction from the element $v_{c s} \sim 1$. So $\left|V_{u s}\right|$ is increased up to its experimental value by $\tilde{t}_{12}^{d} \sim 0.03$ and $\sigma \sim 20^{\circ}$. At the same time $\left|V_{c b}\right|$ is reduced, thanks to the larger value of the asymmetry parameter, $b \sim 12$ which is now not prevented by $V_{u s}$. We can notice that also $\left|V_{u b} / V_{c b}\right|$ is successfully reproduced. The effect of $b$ larger, i.e. of the decreasing of the ratio $Y_{b} / Y_{\tau}$ has in turn induced a bigger $m_{s}$ and a smaller $m_{b}$, both slightly outside their 'reasonable' range. The amount of CP violation from the CKM matrix, though increased thanks to the non-vanishing phase $\sigma$, is still not enough, $B_{K} \sim 2$. The predicted values of the leptonic mixings are good. Accordingly to the approach elucidated in the Sect. 4.4, we have fixed the initial 12 lepton rotation as $\tilde{t}_{12}^{e}=\tilde{t}_{12}^{d}$, i.e. $k_{2}=k_{1}$. Then, as expected, $\sin ^{2} 2 \theta_{12}^{l}$ is strongly reduced below $10^{-2}$ with respect to the case $I$. Notice, that the amount of CP violation in both the quark and lepton mixing matrix is the same as it is controlled by the same phase $\sigma$.

Finally, by comparing the results obtained in the case $I I I$ - where the 22-entry is restored - we conclude that the presence of the 22-entry does not play a significative role - the quality of the fit is quite stable in the two cases. However, the predictions are better. Notice that the right amount of $\mathrm{CP}$ violation is achieved $-B_{K} \sim 0.7$ - as the phase $\sigma$ is now bigger. For 


\begin{tabular}{||c||c|c|c|c||}
\hline \hline $\begin{array}{c}\text { Ansatz B } \\
k_{1,3}=-3, k_{2}=0\end{array}$ & $\begin{array}{c}I . C=0 \\
\left(Y_{t}, b, \xi\right)\end{array}$ & $\begin{array}{c}I I . Y_{c t}=0 \\
\left(Y_{t}, b, \sigma, \tilde{t}_{12}^{d}\right)\end{array}$ & $\begin{array}{c}\text { III. Complete } \\
\left(Y_{t}, b, \xi, \sigma, \tilde{t}_{12}^{d}\right)\end{array}$ & $\begin{array}{c}I V \text {. Spont.CP } \\
\left(Y_{t}, b, \tilde{t}_{12}^{d}\right)\end{array}$ \\
\hline \hline$m_{b}[\mathrm{GeV}]$ & 4.65 & 4.82 & 4.24 & 4.37 \\
\hline$m_{s}[\mathrm{MeV}]$ & 76 & 83 & 87 & 83 \\
\hline$m_{s} / m_{d}$ & 20.3 & 27.3 & 24.4 & 23.0 \\
\hline$\left|V_{u s}\right|$ & 0.2127 & 0.2194 & 0.2196 & 0.2194 \\
\hline$\left|V_{c b}\right|$ & 0.0430 & 0.0575 & 0.0403 & 0.0409 \\
\hline$\left|V_{u b} / V_{c b}\right|$ & 0.014 & 0.102 & 0.094 & 0.099 \\
\hline$\star\left|V_{t d}\right|$ & 0.0097 & 0.011 & 0.0086 & 0.0089 \\
\hline$\star\left|V_{t d} / V_{t s}\right|$ & 0.23 & 0.20 & 0.22 & 0.22 \\
\hline$\star \mathrm{B}_{k}$ & $\infty$ & 0.22 & 0.69 & 0.62 \\
\hline$\star \sin ^{2} 2 \theta_{23}^{l}$ & 0.97 & 0.64 & 1 & 1 \\
\hline$\star \sin ^{2} 2 \theta_{12}^{l}$ & $1.5 \cdot 10^{-2}$ & $1.7 \cdot 10^{-2}$ & $1.3 \cdot 10^{-2}$ & $1.4 \cdot 10^{-2}$ \\
\hline$\star \sin ^{2} 2 \theta_{13}^{l}$ & $1.0 \cdot 10^{-2}$ & $4.5 \cdot 10^{-3}$ & $1.5 \cdot 10^{-2}$ & $1.4 \cdot 10^{-2}$ \\
\hline$\star m_{u} / m_{d}$ & 0.4 & - & - & 0 \\
\hline$\star M_{t} / \sin \beta$ & 172.7 & 195.6 & 172.7 & 172.7 \\
\hline \hline$Y_{t}$ & 0.5 & 0.97 & 0.5 & 0.5 \\
\hline$b$ & 6.8 & 3.5 & 8.8 & 8.2 \\
\hline$\xi$ & 0 & - & $9.7^{\circ}$ & 0 \\
\hline$\sigma$ & - & $95.7^{\circ}$ & $93.5^{\circ}$ & $\pi / 2$ \\
\hline$\tilde{t}_{12}^{d}$ & - & 0.1 & $9.2 \cdot 10^{-2}$ & $9.7 \cdot 10^{-2}$ \\
\hline$\tilde{\chi}_{\min }^{2} \& \chi_{\min }^{2}$ & $11.4 \& 45.2$ & $150.5 \& 112$ & $0.02 \& 0.24$ & $0 \& 0.82$ \\
\hline \hline
\end{tabular}

Table 6: The analysis of the ansatz B. In the fit $I I$ from $Y_{t}=0.97$ it follows $Y_{c t}=1.9 \cdot 10^{-3}$. See also the caption of Table 5 .

the same reason, also $\sin ^{2} 2 \theta_{12}^{l}$ lies exactly in the range required by the MSW solution.

The fact that the best-fit points of the phases, $\xi=\pi, \sigma \approx \pi / 4$, are just integers (or halfintegers) of $\pi$ may suggest that the $\mathrm{CP}$ violating phase is originated by a spontaneous-breaking mechanism. For this reason in the column $I V$ we have performed again the fit fixing the phases $\xi$ and $\sigma$ at the nearest " $\pi$-fold" values of the kind $q \pi(q=1,1 / 2,1 / 4, \cdots)$ as respect to those given by the fit $I I I$. In this case we do not observe any variation. Therefore we can conclude that the ansatz A provides quite a good fit of the quark observables for large 23 asymmetry $b \sim 12$ and $\tilde{t}_{12}^{d} \sim 0.05$ and for $\xi=180^{\circ}, \sigma=45^{\circ}$. The corresponding amount of CP violation appears to be in the right range as indicated by $B_{K} \sim 0.8$. We shall come back to the CP issue below.

Ansatz B We have first to remind that this Clebsch-pattern in its Fritzsch version (vanishing 22 entry, $C=0$ and $b=1$ ) would give $m_{s} / m_{d} \approx 25$ and hence too a small $\left|V_{u s}\right| \approx 0.20$. Let us consider the effect of the non-vanishing 22 entry. From eq. (102) we see that a proper trend can be achieved for $\xi \sim 0$ and $b \geq 6$ so that to maximise $I_{d}$. On the other hand, the moderate asymmetry $b \sim 6-7$ required by the fit implies quite a large $m_{b}$, small $m_{s}$ and consequently also a very tiny $\left|V_{u b} / V_{c b}\right|$. The fit is indeed rather poor (see Table 6, column $I$ ). However, setting to zero the 22-entry and taking $C \neq 0$ spoils completely the fit (Table 


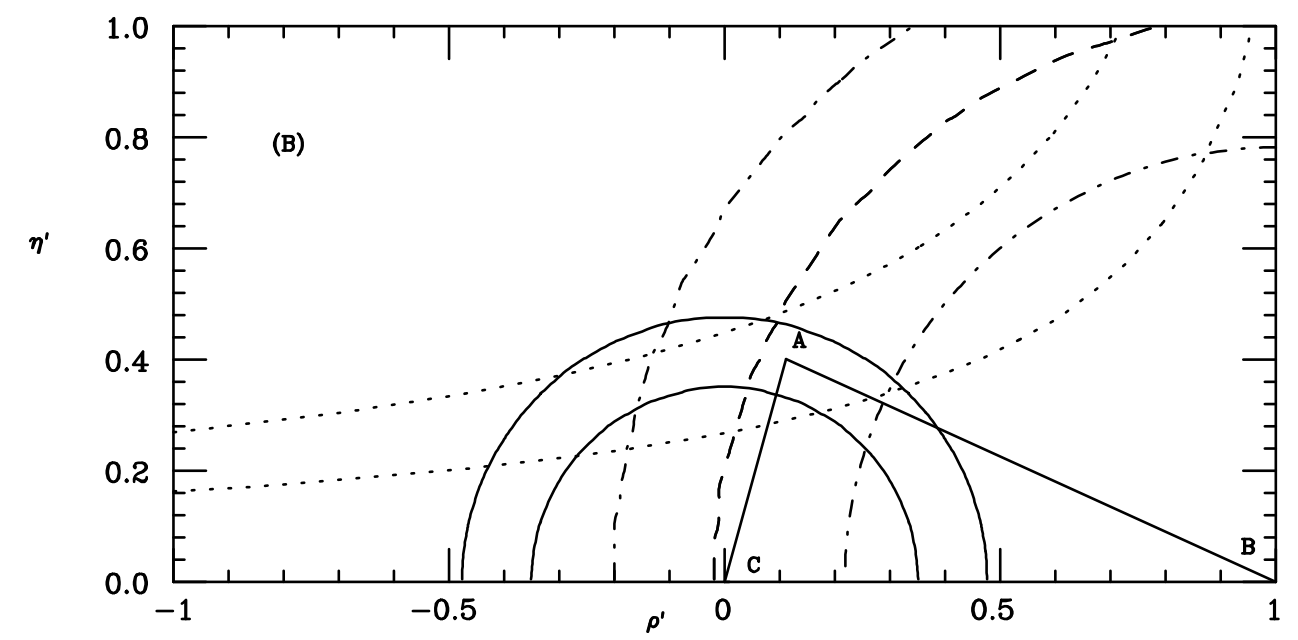

Figure 6: As in Fig. (5) for the ansatzB (case $I I I)$. Here $\lambda=0.2196$ and $A=0.836, R_{b}=0.413 \pm 0.062$, $R_{t}=0.989 \pm 0.207$ (from $\Delta M_{d}$ ) and $R_{t}<1.02\left(\right.$ from $\Delta M_{s}$ ).

6, column $I I)$. Among the CKM elements only $\left|V_{u s}\right|$ and the ratio $\left|V_{u b} / V_{c b}\right|$ get improved thanks to the $\tilde{t}_{12}^{d} \sim 0.1$ rotation. The same rotation is instead less important for $\left|V_{c b}\right|$ which remains too large due to the small asymmetry, $b \sim 3-4$. For the same reason also $m_{s} / m_{d}$ is quite large. In particular as $m_{s} / m_{d}$ is larger than the parameter $Q$, the light-quark mass ratio $m_{u} / m_{d}$ cannot consistently be evaluated. As a whole this case is definitely disfavoured. Remarkably, the leptonic mixing angles as well as $B_{K}$ are not well predicted, too.

Nevertheless, the interplay of the 22-entry with the $(1,3),(3,1)$ entries can offer a satisfactory description as the results in the case $I I I$ show. All the quark quantities are perfectly fitted (notice that both $\tilde{\chi}_{\text {min }}^{2}$ and $\chi_{\text {min }}^{2}$ are $<1$ ). The fit requires $b \sim 9, \tilde{t}_{12}^{d} \sim 0.1$, small $\xi$ and large $\mathrm{CP}$ phase, $\sigma \sim 94^{\circ}$. All the predicted quantities are within their experimental ranges. The 23 leptonic mixing is predicted to be maximal as required by the AN anomaly. The large phase $\sigma$ enforces $\tilde{t}_{12}^{e}=0$ or $k_{2}=0$ and the SN oscillation mixing comes out to be close to the upper limit, $\sin ^{2} 2 \theta_{12}^{l}=1.3 \cdot 10^{-2}$. Indeed, were $\tilde{t}_{12}^{e}=\tilde{t}_{12}^{d}\left(k_{2}=k_{1}\right)$ and $\sigma \sim 90^{\circ}-$ as preferred by the fit - the 12 leptonic mixing $\left|V_{e 2}\right|$ would get further increased (see eq. (117) ), contrary to what happens in the ansatz A. As a further consequence of $\tilde{t}_{12}^{e}=0$ the $\mathrm{CP}$ phase is vanishing in the lepton mixing matrix except for the small contribution driven by the phase $\xi$. Finally in the last column, we have considered the case with $\xi=0$ and $\sigma=\pi / 2$ fixed. The quality of the fit remains very good.

Ansatz C The Fritzsch case for this pattern shows an opposite behaviour as compared to the previous ansatz. Indeed, it is $m_{s} / m_{d} \approx 12 \mathrm{implying}$ too a big $\left|V_{u s}\right| \approx 0.29$. Therefore, in the variant $I$, this can be cured with $\xi>90^{\circ}$ and a large $b$ (see Table 7 , column $I$ ). In this case only $\left|V_{u b} / V_{c b}\right|$ is strongly incompatible with its experimental range. Notice that, for the same asymmetry $b=10, m_{b}$ is smaller as respect to the value obtained in the ansatz $\mathbf{A}$ $(I)$, since as $k_{1}$ is larger as $Z$ becomes smaller. All the predictions are reasonable (except for $\left.B_{K}\right)$. In the second case $(I I)$ all the CKM mixings are well reproduced thanks to the initial 12 rotation, but $Y_{c t}=0$ makes $m_{s} / m_{d}$ below the 'reasonable' range. Finally, the complete model provides quite an excellent fit $(I I I)$. Interestingly, also this ansatzpoints to maximal CP violation $-\sigma \sim 90^{\circ}$. In correspondence of the best-fit parameters all the predicted quantities are in agreement with the present experimental status. We should remark that like in the previous ansatz, the presence of the 22-entry is crucial. Moreover, also in this case the choice $k_{2}=0$ does not induce $\mathrm{CP}$ violation in the lepton mixing matrix. In the last column, we 


\begin{tabular}{|c|c|c|c|c|}
\hline $\begin{array}{c}\text { Ansatz C: } \\
k_{1}=-3, k_{3}=2, k_{2}=0\end{array}$ & $\begin{array}{c}I . C=0 \\
\left(Y_{t}, b, \xi\right)\end{array}$ & $\begin{array}{l}I I . Y_{c t}=0 \\
\left(Y_{t}, b, \sigma, \tilde{t}_{12}^{d}\right)\end{array}$ & $\begin{array}{l}I I I . \text { Complete } \\
\left(Y_{t}, b, \xi, \sigma, \tilde{t}_{12}^{d}\right) \\
\end{array}$ & $\begin{array}{c}I V . \text { Spont.CP } \\
\left(Y_{t}, b, \tilde{t}_{12}^{d}\right) \\
\end{array}$ \\
\hline$m_{b}[\mathrm{GeV}]$ & 4.04 & 4.02 & 4.17 & 4.09 \\
\hline$m_{s}[\mathrm{MeV}]$ & 115 & 111 & 114 & 120 \\
\hline$m_{s} / m_{d}$ & 18.2 & 16.7 & 18.9 & 20.4 \\
\hline$\left|V_{u s}\right|$ & 0.2197 & 0.2196 & 0.2195 & 0.2205 \\
\hline$\left|V_{c b}\right|$ & 0.0378 & 0.0381 & 0.0395 & 0.0381 \\
\hline$\left|V_{u b} / V_{c b}\right|$ & 0.041 & 0.093 & 0.093 & 0.082 \\
\hline$\star\left|V_{t d}\right|$ & 0.0098 & 0.010 & 0.0098 & 0.0093 \\
\hline$\star\left|V_{t d} / V_{t s}\right|$ & 0.27 & 0.28 & 0.26 & 0.25 \\
\hline$\star \mathrm{B}_{k}$ & 9.3 & 0.74 & 0.65 & 0.83 \\
\hline$\star \sin ^{2} 2 \theta_{23}^{l}$ & 0.97 & 0.97 & 1 & 0.99 \\
\hline$\star \sin ^{2} 2 \theta_{12}^{l}$ & $1.2 \cdot 10^{-2}$ & $1.2 \cdot 10^{-2}$ & $1.3 \cdot 10^{-2}$ & $1.3 \cdot 10^{-2}$ \\
\hline$\star \sin ^{2} 2 \theta_{13}^{l}$ & $1.8 \cdot 10^{-2}$ & $1.8 \cdot 10^{-2}$ & $1.4 \cdot 10^{-2}$ & $1.6 \cdot 10^{-2}$ \\
\hline$\star m_{u} / m_{d}$ & 0.6 & 0.7 & 0.5 & 0.4 \\
\hline$\star M_{t} / \sin \beta$ & 172.7 & 172.7 & 187.0 & 181.2 \\
\hline$\overline{Y_{t}}$ & 0.5 & 0.5 & 0.7 & 0.6 \\
\hline$b$ & 10.0 & 10.1 & 8.7 & 9.4 \\
\hline$\xi$ & $107^{\circ}$ & - & $135^{\circ}$ & $\pi$ \\
\hline$\sigma$ & - & $106^{\circ}$ & $93.3^{\circ}$ & $\pi / 2$ \\
\hline $\bar{t}_{12}^{d}$ & - & $7.3 \cdot 10^{-2}$ & $8.6 \cdot 10^{-2}$ & $7.3 \cdot 10^{-2}$ \\
\hline$\tilde{\chi}_{\min }^{2} \& \chi_{\min }^{2}$ & $0.5 \& 15$ & $4.0 \& 0.7$ & $0 \&<0.01$ & $0.02 \& 1.5$ \\
\hline
\end{tabular}

Table 7: The analysis of the ansatz C. In the fit $I I I\left(Y_{t}=0.7\right)$ and $I V\left(Y_{t}=0.6\right)$ it is $Y_{c t}=2.2 \cdot 10^{-3}$ and $2.4 \cdot 10^{-3}$, respectively. See also the caption of Table (5).

have performed a three-parameter fit, setting $\sigma=90^{\circ}$ and $\xi=180^{\circ}$. One could conclude that, by endowing the same pattern with spontaneous breaking of CP-symmetry, the description remains very good.

Finally, we can try to give an overlook at all the three ansätze analysed. The case $\mathbf{A}$ requires the CP-phase $\sigma<90^{\circ}$, whereas both the ansätze $\mathbf{B}$ and $\mathbf{C}$ prefer maximal CP-violation, $\sigma \gtrsim 90^{\circ}$. This should imply in general a different relations between the corresponding CKM elements. For example the three ansätze will show up a different shape of the "unitarity" triangle characterized by the following angles

$$
\alpha \equiv \arg \left(-\frac{V_{t b}^{\star} V_{t d}}{V_{u b}^{\star} V_{u d}}\right), \quad \beta \equiv \arg \left(-\frac{V_{c b}^{\star} V_{c d}}{V_{t b}^{\star} V_{t d}}\right), \quad \gamma \equiv \arg \left(-\frac{V_{u b}^{\star} V_{u d}}{V_{c b}^{\star} V_{c d}}\right) .
$$

Therefore in correspondence of the results of the fits obtained in each ansatz for the complete case $I I I$, we have plotted the unitarity triangle in the $\rho^{\prime}, \eta^{\prime}$ plane (Fig. 6, 7, 8). We recall that $\rho, \eta, A, \lambda$ are the Wolfenstein parameters and $\rho^{\prime}=\rho\left(1-\lambda^{2} / 2\right), \eta^{\prime}=\eta\left(1-\lambda^{2} / 2\right)$. More precisely, the side CB corresponding to $V_{c d} V_{c b}^{\star}$ has been chosen real and rescaled to unit length. Hence the length $\mathrm{CA}$ and $\mathrm{CB}$ in the rescaled triangle, usually denoted by $R_{b}$ and $R_{t}$, 


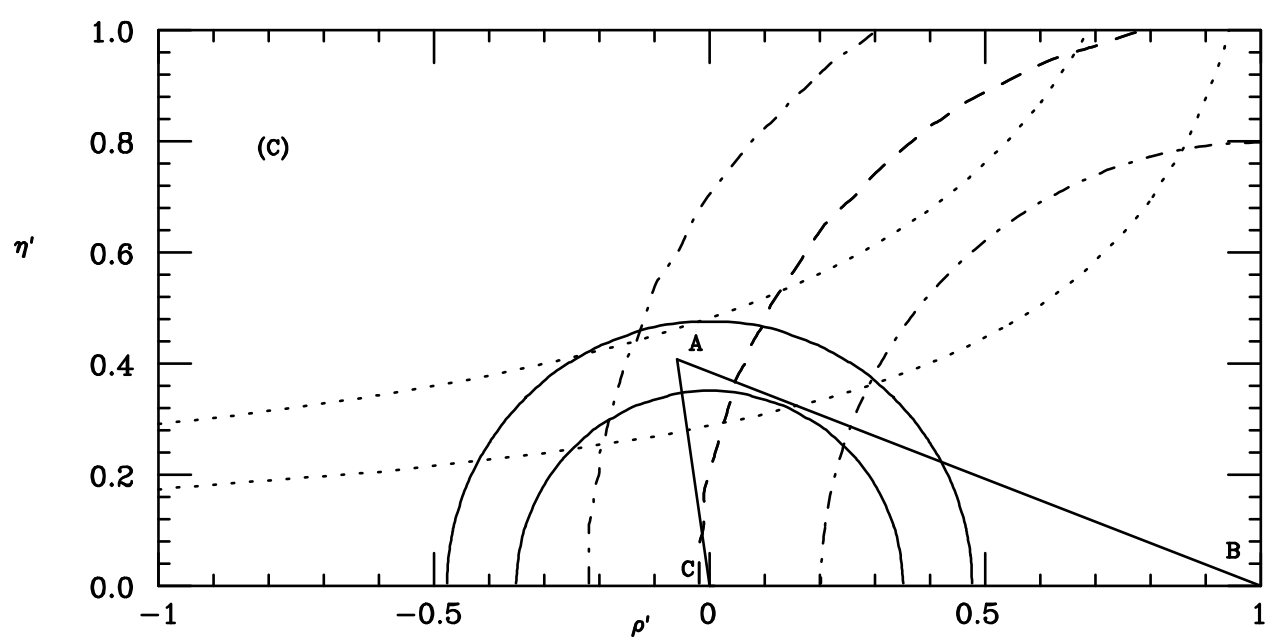

Figure 7: As in Fig. (5) for the ansatz $\mathbf{C}$ (case $I I I)$. Here $\lambda=0.2195$ and $A=0.820, R_{b}=0.413 \pm 0.062$, $R_{t}=1.01 \pm 0.21$ (from $\Delta M_{d}$ ) and $R_{t}<1.02\left(\right.$ from $\left.\Delta M_{s}\right)$.

respectively, are:

$$
\begin{aligned}
& R_{b} \equiv\left|\frac{V_{u d} V_{u b}^{\star}}{V_{c d} V_{c b}^{\star}}\right|=\sqrt{\rho^{\prime 2}+\eta^{\prime 2}}=\left(1-\frac{\lambda^{2}}{2}\right) \frac{1}{\lambda}\left|\frac{V_{u b}}{V_{c b}}\right|, \\
& R_{t} \equiv\left|\frac{V_{t d} V_{t b}^{\star}}{V_{c d} V_{c b}^{\star}}\right|=\sqrt{\left(1-\rho^{\prime}\right)^{2}+\eta^{\prime 2}}=\frac{1}{\lambda}\left|\frac{V_{t d}}{V_{c b}}\right| .
\end{aligned}
$$

Once the parameters $\lambda, A$ have been fixed according to the results of the $I I I$ fit, $\lambda=\left|V_{u s}\right|, A=$ $\left|V_{u s}\right| / \lambda^{2}$, we have depicted the $1-\sigma$ circles of $R_{b}$ from the measured value of $\left|\frac{V_{u b}}{V_{c b}}\right|$ (solid lines), and the circles of $R_{t}$ from the the mass difference $\Delta M_{d, s}$ decribing the strength of the $B_{d, s}^{0}-\bar{B}_{d, s}^{0}$ mixings (dot-dashed and dashed lines, respectively). The values used are $\Delta M_{d}=0.471 \pm 0.016 \mathrm{ps}^{-1}$ and $\Delta M_{s}>12.4 \mathrm{ps}^{-1}$ [1]. In the same plane the hyperbola represent the iso-contours for $B_{K}$ (dotted lines). This overall picture of the CKM elements shows that the ansatzA (see Fig. 5) is characterized by a large angle $\gamma, \gamma>100^{\circ}$, and by a small angle $\beta, \beta \approx 8^{\circ}$. They both appear to be in disagreement with the result reported by the CDF collaboration $\sin 2 \beta=0.79_{-0.44}^{+0.41}$ and with the indirect bounds on $\gamma$ which disfavour $\gamma \gtrsim 100^{\circ}$ [41]. Moreover, the vertex $A$ lies above the region delimited by the circles $R_{t}$ due to the large value of $\left|V_{t d}\right| \sim 0.013$ predicted. On the contrary, the ansatzB provides a consistent scenario where the observed amount of CP-violation can be accounted by the CKM picture (Fig. 6). The same holds for the ansatzC (Fig. 7). Ultimately, a better discrimination of the specific ansatz will be possible when the program of the measurements of the present and future $B$ factories is completed.

\section{Conclusions}

We have considered a particular set of textures for the Yukawa matrices of quarks and leptons which share some features with both the Stech ansatz proposed long time ago [16, 17] and the Fritzsch-like textures suggested in [13]. Our main motivation was to show that they are not only successful from the phenomenological point of view but also grounded and compelling on the theoretical side. We have discussed how these textures could emerge in the context of grand unified theories, in terms of the prototype $S U(5)$ model complemented by the horizontal symmetry $S U(3)_{H}$ (Sect. 2 ). 
The $S U(3)_{H}$ group may seem too 'large' as compared to $U(2)_{H}$. Indeed the latter, providing $a b$ initio the $2+1$ representation structure for the fermion fields, directly singles out the heavy top quark from the lighter fermions of the first and second generation. Namely, the models based on $U(2)_{H}$ 42 invoke the familiar paradigm according to which the third generation has a priori order 1 (tree-level) Yukawa couplings while the small Yukawa constants of the lighter generations emerge from higher-order terms containing the $U(2)_{H}$ symmetry breaking Higgses, with VEVs smaller than the cutoff scale.

However, there are many good points in favour of $S U(3)_{H}$. First, it accounts by itself for three fermion families, and thus can be more predictive than $U(2)_{H}$. Second, the spontaneous breaking features of $S U(3)_{H}$ may turn the Yukawa constants of the low energy theory (MSSM) into dynamical degrees of freedom and fix the inter-family hierarchy in a pretty natural manner. Namely, the third generation becomes heavy $\left(Y_{t} \sim 1\right)$, while the second and first ones become lighter by successively increasing powers of small parameters.

In the general discussion of theoretical issues, we have put forward the following argument: just like the $S U(5)$ theory contains the adjoint Higgs, 24-plet, which, by breaking down $S U(5)$ to $S U(3) \times S U(2) \times U(1)$, does hide the existence of the large GUT symmetry, also $S U(3)_{H}$ may be first broken down to $U(2)_{H}$ by its adjoint Higgs, an octet, which makes the existence of a larger symmetry among all three families less visible.

In particular, the horizontal octet which breaks $S U(3)_{H}$ down to $S U(2)_{H}$, not only diversifies the third family from the lighter ones, but also induces a non-trivial Clebsch structure between the 1-2 and 3 generations in the Yukawa matrices of quarks and leptons. The effect of the latter is twofold. First, it induces the complementary "large - small" (see-saw like) pattern between the neutrino and quark mixing angles, which is indeed exhibited by the observed small value of $V_{c b}$ and the nearly maximal $\nu_{\mu}-\nu_{\tau}$ mixing. Second, it may link the neutrino mass hierarchy to that of the up-quarks in realistic way.

On the other hand, the $S U(5)$ adjoint has proved necessary to break the 'standard' downquark and lepton degeneracy, providing different Clebsch coefficients in the Yukawa matrices. Though its role is not univocal, we have featured three Yukawa patterns characterized by what we consider as the most 'natural' Clebsch factors. In Sect. 3 we have elaborated a consistent $S O(10) \times S U(3)_{H}$ model which, supplemented by the symmetry $\mathcal{G}=U(1)_{A} \times Z_{6} \times \mathcal{R}$, represents an existence proof of the Yukawa texture suggested. In particular, the specific model outlined provides an understanding of the Clebsch pattern of the ansatz $\mathbf{B}$. Remarkably, the same symmetry content of $\mathcal{G}$ also motivates the missing VEV mechanism necessary to achieve the doublet-triplet splitting.

A careful fit of the quark observables demonstrates that all the ansätze $\mathbf{A}, \mathbf{B}$ and $\mathbf{C}$ reproduce quite well the down-quark masses and CKM mixing angles (Sect. 4). What appears very interesting is that for the best-fit ranges of the parameters the predictions for the neutrino mixing angles are in good agreement with the present experimental hints. About the lepton mixing angles, we have to stress that while the outcome for the 23 neutrino mixing is a 'genuine' prediction, in strict connection only with the down-quark sector through the product rule (12), the 12 mixing angle becomes effectively a free parameter fixed by the rotation $\tilde{t}_{12}^{e}$. Should the small angle MSW solution be confirmed in the future, then all the ansätze can account for that with different values of $\tilde{t}_{12}^{e}$. On the contrary, should the 12 neutrino mixing angle be much larger, as indicated by the large-mixing angle regime of the MSW solution, then only the lepton sector would need some refinement.

Interestingly, also the prediction for $\mathrm{CP}$ violation in the quark sector may lie in the observed 
range. In particular, all the ansätze generate a CKM matrix with the correct amount of CP violation. Though the experimental test of $\mathrm{CP}$ violation in the $\mathrm{SM}$ is not yet accomplished, this result may imply that superpartners contributions to $\varepsilon_{K}$ must be adequately suppressed [38] and it would be interesting to study in the theoretical models presented here the implications of the horizontal symmetry on the SUSY spectrum.

\section{Acknowledgements}

We thank Andrea Brignole and Denis Comelli for useful discussions. This work is partially supported by the MURST research grant of national interest "Astroparticle Physics".

\section{Appendix A}

Consider the following superpotential terms including antisextet superfield $S=S^{i j}$ and its sextet partner $\bar{S}=\bar{S}_{i j}$ :

$$
W_{S}=-\mu S \bar{S}+S^{3}+\bar{S}^{3},
$$

where, to be explicit, $S^{3}=\frac{1}{3} \varepsilon_{i j k} \varepsilon_{l m n} S^{i l} S^{j m} S^{k n}=\operatorname{det} S$ (similarly for $\bar{S}^{3}$ ), and order 1 coupling constants are absorbed. Observe that this superpotential is manifestly invariant under $Z_{3}$ symmetry: $S \rightarrow \exp \left(i \frac{2 \pi}{3}\right) S$ and $\bar{S} \rightarrow \exp \left(-i \frac{2 \pi}{3}\right) \bar{S}$.

Without loss of generality, the VEV of $S$ can be chosen in the diagonal form, $\langle S\rangle=$ $\operatorname{Diag}\left(\mathcal{S}_{1}, \mathcal{S}_{2}, \mathcal{S}_{3}\right)$. Then the condition of vanishing $F$-terms $F_{S}, F_{\bar{S}}=0$ implies that $\langle\bar{S}\rangle$ is also diagonal, $\langle\bar{S}\rangle=\operatorname{Diag}\left(\overline{\mathcal{S}}_{1}, \overline{\mathcal{S}}_{2}, \overline{\mathcal{S}}_{3}\right)$, and

$$
\begin{array}{ll}
\mathcal{S}_{1} \mathcal{S}_{2}=\mu \overline{\mathcal{S}}_{3}, & \overline{\mathcal{S}}_{1} \overline{\mathcal{S}}_{2}=\mu \mathcal{S}_{3}, \\
\mathcal{S}_{1} \mathcal{S}_{3}=\mu \overline{\mathcal{S}}_{2}, & \overline{\mathcal{S}}_{1} \overline{\mathcal{S}}_{3}=\mu \mathcal{S}_{2}, \\
\mathcal{S}_{2} \mathcal{S}_{3}=\mu \overline{\mathcal{S}}_{1}, & \overline{\mathcal{S}}_{2} \overline{\mathcal{S}}_{3}=\mu \mathcal{S}_{1} .
\end{array}
$$

In the exact supersymmetric limit the VEV pattern of $S$ and $\bar{S}$ is not fixed unambiguously and there remain flat directions which represent a two-parameter vacuum valley. In other words, the six equations (128) reduce to four conditions:

$$
\mathcal{S}_{1} \overline{\mathcal{S}}_{1}=\mathcal{S}_{2} \overline{\mathcal{S}}_{2}=\mathcal{S}_{3} \overline{\mathcal{S}}_{3}=\mu^{2}, \quad \mathcal{S}_{1} \mathcal{S}_{2} \mathcal{S}_{3}=\mu^{3} ; \quad \overline{\mathcal{S}}_{1} \overline{\mathcal{S}}_{2} \overline{\mathcal{S}}_{3}=\mu^{3}
$$

while the others are trivially fulfilled (the last in eq. (129) is not independent, it just follows from the others). Thus, in principle the eigenvalues $\mathcal{S}_{1,2,3}$ can be different from each other, say $\mathcal{S}_{3}>\mathcal{S}_{2}>\mathcal{S}_{1}$. Then eqs. (128) imply that $\overline{\mathcal{S}}_{1,2,3}$ should have an inverse hierarchy, $\overline{\mathcal{S}}_{3}<\overline{\mathcal{S}}_{2}<\overline{\mathcal{S}}_{1}$. More precisely, we have $\overline{\mathcal{S}}_{1}: \overline{\mathcal{S}}_{2}: \overline{\mathcal{S}}_{3}=\mathcal{S}_{1}^{-1}: \mathcal{S}_{2}^{-1}: \mathcal{S}_{3}^{-1}$.

The flat directions of the VEVs can be fixed from the soft supersymmetry breaking terms. The relevant ones are D-terms: $: 11$

$$
\mathcal{L}=-\int d^{4} \theta z \bar{z}\left[\alpha \operatorname{Tr} S^{\dagger} S+\frac{\beta}{M^{2}}\left(\operatorname{Tr} S^{\dagger} S\right)^{2}+\frac{\gamma}{M^{2}} \operatorname{Tr}\left(S^{\dagger} S S^{\dagger} S\right)+\ldots\right]+[S \rightarrow \bar{S}]
$$

\footnotetext{
${ }^{31}$ The F-terms $\sim \int d^{2} \theta z W$ are not relevant for the VEV orientation as far as they just repeat the holomorphic invariants like $S \bar{S}$ and $\operatorname{det} S$ whose values are already fixed by the conditions (129).
} 
having a similar form for $S$ and $\bar{S}$. Here $z=m \theta^{2}$ and $\bar{z}=m \bar{\theta}^{2}$ are supersymmetry breaking spurions, with $m \sim 1 \mathrm{TeV}$. The cutoff scale $M$ is taken as the flavour scale, i.e. the same as the one in superpotential terms (24), and we assume that $M \gg \mu$. Therefore, for $S_{1,2,3}$ these terms translate explicitly into the following scalar potential:

$\alpha m^{2}\left(\left|\mathcal{S}_{1}\right|^{2}+\left|\mathcal{S}_{2}\right|^{2}+\left|\mathcal{S}_{3}\right|^{2}\right)+\beta \frac{m^{2}}{M^{2}}\left(\left|\mathcal{S}_{1}\right|^{2}+\left|\mathcal{S}_{2}\right|^{2}+\left|\mathcal{S}_{3}\right|^{2}\right)^{2}+\gamma \frac{m^{2}}{M^{2}}\left(\left|\mathcal{S}_{1}\right|^{4}+\left|\mathcal{S}_{2}\right|^{4}+\left|\mathcal{S}_{3}\right|^{4}\right)+\left[\left|\mathcal{S}_{k}\right| \rightarrow\left|\overline{\mathcal{S}}_{k}\right|\right]$

The stability of the potential implies that $\beta>0$ and $\gamma>-\beta$, whereas $\alpha$ can be positive or negative. In the former case the minimization of the potential (131), under the conditions (129), would imply that $\mathcal{S}_{1}=\mathcal{S}_{2}=\mathcal{S}_{3}=\mu$, i.e. no hierarchy between the fermion families. In the latter case, however, the largest eigenvalue of $S$ and $\bar{S}$, respectively $\mathcal{S}_{3}$ and $\overline{\mathcal{S}}_{1}$, grow up above the typical VEV size $\mu$ and reach values of the order of the cutoff scale $M$ :

$$
\mathcal{S}_{3}, \overline{\mathcal{S}}_{1}=\left(\frac{\alpha}{2(\beta+\gamma)}\right)^{1 / 2} M
$$

that is $\mathcal{S}_{3}, \overline{\mathcal{S}}_{1} \sim M$. Then it follows from (129) that

$$
\mathcal{S}_{2}, \overline{\mathcal{S}}_{2}=\mu=\varepsilon \mathcal{S}_{3}, \quad \mathcal{S}_{1}, \overline{\mathcal{S}}_{3}=\frac{\mu^{2}}{\mathcal{S}_{3}}=\varepsilon^{2} \mathcal{S}_{3},
$$

where $\varepsilon=\mu / \mathcal{S}_{3} \sim \mu / M$.

Let us assume now that the theory contains three triplets superfields $A_{n}=A_{n i}$, and their partners $\bar{A}_{n}=\bar{A}_{n}^{i}, n=1,2,3$. One can incorporate them by the following terms in the superpotential:

$$
W_{A}=-\sum_{n} \mu_{n} A_{n} \bar{A}_{n}+A_{1} A_{2} A_{3}+\bar{A}_{1} \bar{A}_{2} \bar{A}_{3}
$$

where order 1 coupling constants are understood and $A_{1} A_{2} A_{3} \equiv \varepsilon^{i j k} A_{1 i} A_{2 j} A_{3 k}$. For simplicity, we shall take all masses $\mu_{n}$ equal, $\mu_{1,2,3}=\mu^{\prime}<M$. In addition, by assuming that the triplets have $Z_{3}$ charges different from that of $S$, we do not include terms like $S A^{2} \equiv S^{i j} A_{i} A_{j}$. In this way, in the exact supersymmetric limit the ground state has a continuous degeneracy (flat direction) related to unitary transformations $A_{n} \rightarrow U A_{n}$ with $U \subset S U(3)_{A}$. In other terms, the superpotential $W=W_{S}+W_{A}$ has an accidental global symmetry $S U(3)_{S} \times S U(3)_{A}$, with the two $S U(3)$ factors independently transforming the two sets of horizontal superfields, $S$ and A.

Similarly to the case of the Higgs fields $S, \bar{S}$, now the conditions $F_{A}, F_{\bar{A}}=0$ can only fix the values of holomorphic invariants $A_{n} \bar{A}_{n}$ and $A_{1} A_{2} A_{3}$. Namely, by unitary transformation $A_{n} \rightarrow U A_{n}\left(U \subset S U(3)_{A}\right)$, one can choose a basis where the VEV of $A_{1}$ points towards the first component. Then we see that in this basis the fields $A_{2}$ and $A_{3}$ should have the VEVs towards the second and third components. In other words, in this basis the triplets have the $\operatorname{VEVs}\left\langle A_{n i}\right\rangle=\delta_{n}^{i} \mathcal{A}_{i}$, satisfying the following equations:

$$
\mathcal{A}_{1} \overline{\mathcal{A}}_{1}=\mathcal{A}_{2} \overline{\mathcal{A}}_{2}=\mathcal{A}_{3} \overline{\mathcal{A}}_{3}=\mu^{\prime 2}, \quad \mathcal{A}_{1} \mathcal{A}_{2} \mathcal{A}_{3}=\mu^{\prime 3}, \quad \overline{\mathcal{A}}_{1} \overline{\mathcal{A}}_{2} \overline{\mathcal{A}}_{3}=\mu^{\prime 3} .
$$

The three VEVs can be different and one can take, say $\mathcal{A}_{1}>\mathcal{A}_{2}>\mathcal{A}_{3}$. The hierarchy between the latter can be fixed by soft D-terms

$$
\mathcal{L}=-\int d^{4} \theta z \bar{z}\left[\alpha^{\prime} \sum_{n} A_{n}^{\dagger} A_{n}+\frac{\beta^{\prime}}{M^{2}} \sum_{m n}\left(A_{m}^{\dagger} A_{m}\right)\left(A_{n}^{\dagger} A_{n}\right)+\frac{\gamma^{\prime}}{M^{2}} \sum_{m \neq n}\left(A_{m}^{\dagger} A_{n}\right)\left(A_{n}^{\dagger} A_{m}\right)\right]+\left[A_{n} \rightarrow \bar{A}_{n}\right]
$$


with $\alpha^{\prime}<0$. Then, similarly to what happened to the sextets, the largest VEV rises up to the cutoff scale $M: \mathcal{A}_{1}, \overline{\mathcal{A}}_{3} \sim M$, while $\mathcal{A}_{2}, \overline{\mathcal{A}}_{2} \sim \varepsilon^{\prime} M$ and $\mathcal{A}_{3}, \overline{\mathcal{A}}_{1} \sim \varepsilon^{\prime 2} M$, with $\varepsilon^{\prime} \sim \mu^{\prime} / M \sim$ $\left(\mu^{\prime} / \mu\right) \varepsilon$.

As for the relative orientation of the sextet and triplet VEVs, these will also be fixed from the soft D-terms:

$$
\mathcal{L}=-\int d^{4} \theta z \bar{z} \frac{\delta}{M^{2}} \sum_{n}\left(A_{n}^{\dagger} S S^{\dagger} A_{n}\right)+\left[S \rightarrow \bar{S}, A_{n} \rightarrow \bar{A}_{n}\right]
$$

Indeed, for positive $\delta$ the favoured orientation between the $S$ and $A$ bases, which minimizes the energy of the ground state, corresponds to $U=1$.

The above can be interpreted in the following manner. In the view of operators like (24), the MSSM Yukawa constants become dynamical degrees of freedom. In particular, the operator $\mathcal{O}_{u}$ in (24) implies

$$
\mathbf{Y}_{u}=\left(\begin{array}{ccc}
Y_{u} & 0 & 0 \\
0 & Y_{c} & 0 \\
0 & 0 & Y_{t}
\end{array}\right) \sim \frac{\left\langle S^{i j}\right\rangle}{M}=\frac{1}{M}\left(\begin{array}{ccc}
\mathcal{S}_{1} & 0 & 0 \\
0 & \mathcal{S}_{2} & 0 \\
0 & 0 & \mathcal{S}_{3}
\end{array}\right)
$$

In the exact supersymmetric limit the values of the constants $Y_{u, c, t}$ are not fixed - they have flat directions and eq. (129) translates into the constraint $Y_{u} Y_{c} Y_{t}=\varepsilon^{3}$. However, we have shown that soft supersymmetry breaking terms could naturally split the Yukawa constants so that $Y_{t} \sim 1$, while $Y_{c} \sim \varepsilon$ and $Y_{u} \sim \varepsilon^{2}$, which perfectly reflects the observed pattern if $\varepsilon \sim 5 \cdot 10^{-3}$. (For discussions on dynamical $Y_{t}$ in supersymmetry see also ref. [43].)

As for the triplet VEVs, in view of the operator $\mathcal{O}(24)$ they provide off-diagonal Yukawa entries

$$
\mathbf{A}=\left(\begin{array}{ccc}
0 & A_{3} & A_{2} \\
-A_{3} & 0 & A_{1} \\
-A_{2} & -A_{1} & 0
\end{array}\right) \sim \sum_{n} \frac{\left\langle A_{n}^{i j}\right\rangle}{M}=\frac{1}{M}\left(\begin{array}{ccc}
0 & \mathcal{A}_{3} & \mathcal{A}_{2} \\
-\mathcal{A}_{3} & 0 & \mathcal{A}_{1} \\
-\mathcal{A}_{2} & -\mathcal{A}_{1} & 0
\end{array}\right)
$$

with the constraint $A_{1} A_{2} A_{3}=\varepsilon^{\prime 3}$ in the supersymmetric limit, while the soft breaking terms can dynamically fix their values as $A_{1} \sim 1, A_{2} \sim \varepsilon^{\prime}$ and $A_{3} \sim \varepsilon^{\prime 2}$. In the context of our work it is clear that such a hierarchy of the off-diagonal entries in $\mathbf{Y}_{d, e}$ is also well suited for the observed fermion mass and mixing pattern if $\varepsilon^{\prime} \sim 0.1$.

One has to remark that the above considerations are literally valid if the horizontal symmetry is global. For the case of local $S U(3)_{H}$, apart from F-flatness conditions, there are eight additional conditions: the gauge $D$ terms $D_{a}=\sum_{n} X^{\dagger} T^{a} X$, should vanish on the vacuum configuration $\left(T^{a}\right.$ are $S U(3)_{H}$ generators, $\left.a=1, . .8\right)$. Although this does not occur for the obtained VEV pattern of $S$ and $A_{n}$, one can easily imagine the theory to contain additional "spectator" superfields in different representations of $S U(3)_{H}$ and their VEVs are oriented so that to cancel the contributions of $\langle S\rangle$ and $\langle A\rangle$ in gauge D-terms of $S U(3)_{H}$. In order not to affect the obtained solutions for $S$ and $A_{n}$, these extra superfields should not couple to the latter in the superpotential.

\section{Appendix B}

Let us assume now that the considered theory has an exact $\mathrm{CP}$ invariance. In other words, we can choose a superfield basis where all coupling constants in the theory are real, 
including the coupling constants with the matter superfields, i.e. those in the terms (24). Applied to the superpotential (127) this means that the dimensional parameter $\mu$ as well as the understood constants at $S^{3}$ and $\bar{S}^{3}$ are real. Without losse of generality, by means of a $S U(3)_{H}$ transformation, one can chose a basis where $\mathcal{S}_{3}$ and $\mathcal{S}_{2}$ are real and positive. Then, since eq. (129) tells us that the VEV products $\mathcal{S}_{1} \mathcal{S}_{2} \mathcal{S}_{3}=\mu^{3}$ is real, also the component $\mathcal{S}_{1}$ should be real. The same consideration is true for the VEVs of triplets $A_{n}$. Therefore, no CP-violation can occur in this case.

Let us now consider an alternative superpotential for triplets (134) not containing the mass terms but invoking instead an auxiliary singlet $I$ :

$$
W_{A}=\kappa_{n} I A_{n} \bar{A}_{n}-A_{1} A_{2} A_{3}-\bar{A}_{1} \bar{A}_{2} \bar{A}_{3}+3 \Lambda^{2} I+I^{3}
$$

where $\Lambda$ is a dimensional parameter, $\kappa_{n}$ are coupling constants and order one constants are also understood in other terms. Applied to this superpotential, CP invariance means that $\kappa_{n}$ as well as $\Lambda^{2}$ are real. For simplicity, let us assume again that all $\kappa_{n}$ are equal, $\kappa_{n}=\kappa$, and $\kappa \ll 1$.

It is clear, that the $F$-term conditions $F_{A}, F_{\bar{A}}=0$ are the same as in (135) apart from the fact that the mass scale $\mu^{\prime}$ should be substituted by $\kappa \mathcal{I}$, where $\mathcal{I}=\langle I\rangle$. The latter is then fixed by the condition $F_{I}=0$ given as

$$
\Lambda^{2}+\mathcal{I}^{2}+\frac{1}{3} \kappa \sum_{k=1}^{3} \mathcal{A}_{k} \overline{\mathcal{A}}_{k}=\Lambda^{2}+\left(1+\kappa^{3}\right) \mathcal{I}^{2}=0
$$

Hence, $\mathcal{I}^{2} \simeq-\Lambda^{2}$, and if $\Lambda^{2}$ is positive, then $\mathcal{I}$ should be imaginary and so we obtain spontaneous $\mathrm{CP}$ violation. In other words, imaginary $\mathcal{I}$ means that the $\mathrm{CP}$-odd component of the superfield $I$ acquires a VEV.

As a result, the 'induced' mass $\mu^{\prime}=\kappa \mathcal{I}$ and so the VEV product $\mathcal{A}_{1} \mathcal{A}_{2} \mathcal{A}_{3}=\mu^{\prime 3}$ are also imaginary. Therefore, at least one of the VEVs $\mathcal{A}_{1,2,3}$ should be complex and thus would induce the corresponding phase in the fermion Yukawa matrices.

To fix the point, we have to find the relative phase orientation between the VEVs of $S$ and $A$. These should be determined by soft terms like

$$
\mathcal{L}=-\int d^{4} \theta z \bar{z} \frac{\zeta}{M^{2}}\left(S^{\dagger} S^{\dagger} A_{n} A_{n}+\text { h.c. }\right)
$$

etc., with $\zeta$ being a real constant. In particular, the above term induces the following couplings in the Higgs potential:

$$
\zeta \frac{m^{2}}{M^{2}}\left(\mathcal{S}_{3} \mathcal{S}_{2} \mathcal{A}_{1}^{2}+\mathcal{S}_{3} \mathcal{S}_{1} \mathcal{A}_{2}^{2}+\mathcal{S}_{2} \mathcal{S}_{1} \mathcal{A}_{3}^{2}\right)+\text { h.c. }
$$

We see that for $\zeta>0$ the minimization of the ground state energy requires that in the basis where $\mathcal{S}_{k}$ are real and positive, $\mathcal{A}_{1,2,3}^{2}<0$, i.e. all triplets have imaginary VEVs. For $\zeta<0$ we see instead that $\mathcal{A}_{1,2}$ prefer to be real. From the couplings (143) also the smallest VEV $\mathcal{A}_{3}$ would prefer to be real; however it is forced to have a complex phase $\pi / 2$ by the constraint that the product $\mathcal{A}_{1} \mathcal{A}_{2} \mathcal{A}_{3}$ is imaginary. Both these cases can be of interest for the CP-breaking phases of the fermion Yukawa textures discussed in this work. 


\section{References}

[1] SuperKamiokande Collaboration, Y. Fukuda et al., Phys. Rev. Lett. 81 (1998) 1562; 2016; M. Nakahata, in TAUP 99, $V$ Int. Workshop on Topics in Astroparticle and Underground Physics (Paris, Sept. 1999), to appear; Transparencies available at http://taup99.in2p3.fr/TAUP99/;

M. Takita, in Cosmo 99, Int. Workshop on Particle Physics and the Early Universe, (Trieste, Sept. 1999); Transparencies available at http://www.ictp.it/ cosmo99/.

[2] For a review on the present status see e.g. G.L. Fogli in TAUP 99, V Int. Workshop on Topics in Astroparticle and Underground Physics (Paris, Sept. 1999), to appear; Transparencies available at http://taup99.in2p3.fr/TAUP99/;

N. Fornengo, M. C. Gonzalez-Garcia and J. W. Valle, hep-ph/0002147.

[3] See e.g. Y. Suzuki, in Lepton-Photon '99 Proc. of the XIX Int. Symposium on Photon and Lepton Interactions at High Energies, (Stanford, California - USA, 1999) to appear; transparencies available at http://lp99.slac.stanford.edu.

[4] J. Bahcall, P. Krastev and A. Smirnov, Phys. Rev. D58 (1998) 096016;

M. Gonzalez-Garcia, P. de Holanda, C. Pena-Garay and J.W.F. Valle, hep-ph/9906469.

[5] J. Bahcall, P. Krastev, A. Smirnov, Phys. Rev. D60 (1999) 093001; hep-ph/9911248.

[6] S.P. Mikheyev and A.Yu. Smirnov, Yad. Fiz. 42 (1985) 1441;

L. Wolfenstein, Phys. Rev. D17 (1978) 2369.

[7] S. Weinberg, Phys. Rev. Lett. 43 (1979) 1566; R. Barbieri, J. Ellis and M.K. Gaillard, Phys. Lett. 90B (1980) 249; E. Akhmedov, Z. Berezhiani and G. Senjanović, Phys. Rev. Lett. 69 (1992) 3013.

[8] M. Gell-Mann, P. Ramond and R. Slansky, in Supergravity, eds. D. Freedman et al., NorthHolland, Amsterdam, 1979; T. Yanagida, Prog. Th. Phys. B135 (1979) 66; R. Mohapatra and G. Senjanović, Phys. Rev. Lett. 44 (1980) 912.

[9] Particle Data Group, Eur. Phys. J. C3 (1998) 1.

[10] For a review see e.g. Z. Berezhiani, Proc. 1995 Summer School, in: High Energy Physics and Cosmology, E. Gava et al. (Eds.), The ICTP Series in Theoretical Physics - vol. 12, World Scientific, Singapore, p. 618 (hep-ph/9602325); R.N. Mohapatra, Lectures at ICTP Summer School in Particle Physics, Trieste, Italy, June 1999 (hep-ph/9911272).

[11] Z. Berezhiani and A. Rossi, Phys. Lett. B367 (1996) 219;

M. Drees, S. Pakvasa, X. Tata, T. ter Veldhuis, Phys. Rev. D57 (1998) 5335;

R. Barbieri, L. Hall, D. Smith, A. Strumia and N. Weiner, JHEP 12 (1998) 017;

R. Barbieri, L. Hall and A. Strumia, Phys. Lett. B 445 (1999) 407;

G. Altarelli and F. Feruglio, Phys. Lett. B439 (1998) 112; JHEP 9811 (1998) 021;

H. Fritzsch and Z. Xing, Phys. Lett. B440 (1998) 313;

B. Mukhopadhya, S. Roy, F. Vissani, Phys. Lett. B443 (1998) 191;

Y. Grossman, Y. Nir and Y. Shadmi, JHEP 10 (1998) 007;

M. Gomez, G. Leontaris, S. Lola, J. Vergados, Phys. Rev. D59 (1999) 116009; 
S. Lola and G.G. Ross, Nucl. Phys. B553 (1999) 81;

M. Tanimoto, Phys. Rev D59 (1999) 017304; Phys. Lett. B456 (1999) 220;

G. Altarelli, F. Feruglio, I. Masina, hep-ph/9907532;

M.A. Diaz, J. Ferrandis and J.W. Valle, hep-ph/9909212;

D.A. Ryzhik, K.A. Ter-Martirosian, hep-ph/9909424;

E.K. Akhmedov, G.C. Branco, M.N. Rebelo, hep-ph/9912205. For an alternative approach to the generation of maximal neutrino mixing see J.C. Romao, M.A. Diaz, M. Hirsch, W. Porod and J.W. Valle, Phys. Rev. D 61 (2000) 071703.

[12] Z. Berezhiani, Z. Tavartkiladze, Phys. Lett. B409 (1997) 220; ibid. B396 (1997) 150;

C. Albright, K.S. Babu and S. Barr, Phys. Rev. Lett. 81 (1998) 1167;

G. Altarelli and F. Feruglio, Phys. Lett. B451 (1999) 388;

T. Blazek, S. Raby, K. Tobe, Phys. Rev. D 60 (1999) 113001, hep-ph/9912482.

[13] Z. Berezhiani and A. Rossi, JHEP 9903 (1999) 002, hep-ph/9811447; Nucl. Phys. (Proc. Suppl.) B 81 (2000) 346, hep-ph/9907397.

[14] CHOOZ Collaboration, M. Apolonio et al., Phys. Lett. B420 (1998) 397; ibid. B466 (1999) 415. See also the initial results by Palo Verde experiment, F. Boehm et al., hep-ex/9912050.

[15] H. Fritzsch, Nucl. Phys. B155 (1979) 189.

[16] B. Stech, Phys. Lett. B130 (1983) 189.

[17] Z. Berezhiani and J. Chkareuli, JETP Lett. 35 (1982) 612; Yad. Fiz. 37 (1983) 1043 [Sov. J. Nucl. Phys. 37 (1983) 618].

[18] J. Chkareuli, JETP Lett. 32 (1980) 671.

[19] Z. Berezhiani and E. Nardi, Phys. Lett. B355 (1995) 199; Phys. Rev. D 52 (1995) 3087.

[20] Z. Berezhiani and M. Khlopov, Yad. Fiz. 51 (1990) 1157, ibid. 51 (1990) 1479.

[21] Z. Berezhiani, G. Dvali, M. Jibuti and J. Chkareuli, Proc. Int. Seminar "Quarks 86", Tbilisi 1986, A. Tavkhelidze et al. (Eds.), INR Press, Moscow, 1986, p. 209.

[22] C.D. Frogatt and H.B. Nielsen, Nucl. Phys. B147 (1979) 277;

Z. Berezhiani, Phys. Lett. B129 (1983) 99; Phys. Lett. B150 (1985) 177;

S. Dimopoulos, Phys. Lett. B129 (1983) 417.

[23] A. Anselm and Z. Berezhiani, Nucl. Phys. B484 (1997) 97.

[24] H. Georgi and C. Jarlskog, Phys. Lett. B86 (1979) 297;

J. Harvey, P. Ramond and D. Reiss, Phys. Lett. B92 (1980) 309;

S. Dimopoulos, in ref. [22];

G. Anderson, S. Dimopoulos, L.J. Hall, S. Raby and G. Starkman, Phys. Rev. D 49 (1994) 3660 .

[25] Z. Berezhiani and G. Dvali, Sov. Phys. Lebedev Inst. Reports 5 (1989) 44;

R. Barbieri, G. Dvali and M. Moretti, Phys. Lett. B312 (1993) 137;

R. Barbieri et al., Nucl. Phys. B432 (1994) 49; 
Z. Berezhiani, Phys. Lett. B355 (1995) 481;

Z. Berezhiani, C. Csaki and L. Randall, Nucl. Phys. B444 (1995) 61;

G. Dvali and S. Pokorski, Phys. Rev. Lett. 78 (1997) 807.

[26] J. Chkareuli and A. Kobakhidze, Phys. Lett. B407 (1997) 234;

J. Chkareuli, I. Gogoladze and A. Kobakhidze, Phys. Rev. Lett. 80 (1998) 912;

J. Chkareuli, C.D. Froggatt, I. Gogoladze, A. Kobakhidze, hep-ph/0003007.

[27] S. Dimopoulos and F. Wilczek, Report No. NSF-ITP-82-07 (unpublished)

[28] K.S. Babu and S.M. Barr, Phys. Rev. D48 (1993) 5354.

[29] Z. Berezhiani, Z. Tavartkiladze, ref. [12]

[30] M. Dine, N. Seiberg and E. Witten, Nucl. Phys. B 289 (1987) 585;

J. Atick, L. Dixon and A. Sen, Nucl. Phys. B 292 (1987) 109;

M. Dine, A. Ichinose and N. Seiberg, Nucl. Phys. B 293 (1987) 253.

[31] S. Barr, Phys. Rev. D 24 (1981) 1895;

Z. Berezhiani, Yad. Fiz. 42 (1985) 1309 [Sov. J. Nucl. Phys. 42 (1985) 825];

G. Dvali and S. Pokorski, Phys. Lett. B379 (1996) 134.

[32] V. Barger, M. S. Berger and P. Ohmann, Phys. Rev. D47 (1993) 2038.

[33] P. Chankowski and Z. Pluciennik, Phys. Lett. B316 (1993) 312;

C.N. Leung, K.S. Babu and J. Pantaleone, ibid. B319 (1993) 191;

A. Brignole, H. Murayama and R. Rattazzi, ibid. B335 (1994) 345;

A. Smirnov and F. Vissani, ibid. B341 (1994) 173.

[34] J. Ellis and S. Lola, Phys. Lett. B458 (1999) 310;

N. Haba and N. Okamura, hep-ph/9810471;

J.A. Casas, J.R. Espinosa, A. Ibarra and I. Navarro, hep-ph/9910420.

[35] For a review and references, see for instance A.J. Buras and R. Fleischer, hep-ph/9704376;

in Heavy Flavours II, World Scientific (1997), Eds. A.J. Buras and M. Lindner.

[36] Z. Berezhiani, Phys. Lett. B417 (1998) 287; Nucl. Phys. B (Proc. Suppl.) 52A (1997) 153, Proc. Fourth Int. Conference SUSY '96, eds. R.N. Mohapatra and A. Rasin, North-Holland (hep-ph/9607363).

[37] A. Kostelecky, L.J. Hall and S. Raby, Nucl. Phys. B267 (1986) 415;

R. Barbieri and L.J. Hall, Phys. Lett. B338 (1994) 212.

[38] See e.g. Y. Grossman, Y. Nir and R. Rattazzi, in Heavy Flavours II, World Scientific (1997), Eds. A.J. Buras and M. Lindner, p. 755, hep-ph/9701231; Y. Nir, hep-ph/9911321,

[39] H. Leutwyler, Talk given at the Conference on Fundamental Interactions of Elementary Particles, ITEP, Moscow, Russia, 1995, hep-ph/9602255.

[40] The Neutrino Group of the Prospective Study on $\mu^{+} \mu^{-}$Colliders, B. Autin et al., preprint CERN-SPSC/98-30, October 1998;

see also A. de Rujula, M. Gavela and P. Hernandez, Nucl. Phys. B547 (1999) 21;

V. Barger, S. Geer, R. Raja and K. Whisnant, hep-ph/9911524. 
[41] F. Parodi, P. Roudeau and A. Stocchi, hep-ex/9903063;

see also the theoretical discussion by M. Neubert, hep-ph/9904321.

[42] A. Pomarol and D. Tommasini, Nucl. Phys. B466 (1996) 3;

R. Barbieri, G. Dvali and L.J. Hall, Phys. Lett. B337 (1996) 76;

R. Barbieri and L.J. Hall, Nuovo. Com. 110A (1996) 1;

R. Barbieri, L.J. Hall, S. Raby, A. Romanino, Nucl. Phys. B493 (1997) 3.

[43] P.Binetruy and E. Dudas, Phys. Lett. B338 (1994) 23;

C. Kounnas, I. Pavel, G. Ridolfi and F. Zwirner, Phys. Lett. B354 (1995) 322. 